October 29, $2018 \quad 8: 57 \quad$ jwbsb1arxiv2

\title{
Existence and Approximation of a (Regularized) Oldroyd-B Model
}

\author{
John W. Barrett \\ Department of Mathematics, Imperial College London, \\ London SW7 2AZ, UK \\ jwb@ic.ac.uk \\ Sébastien Boyaval \\ CERMICS, Ecole Nationale des Ponts et Chaussées (ParisTech/Université Paris-Est) \\ 688 avenue Blaise Pascal, Cité Descartes, 77455 Marne-la-Vallée Cedex 2, France \\ sebastien.boyaval@cermics.enpc.fr \\ Present address: Laboratoire d'hydraulique Saint-Venant, \\ Université Paris-Est (Ecole des Ponts ParisTech) EDF RED, \\ 6 quai Watier, 78401 Chatou Cedex, France
}

\begin{abstract}
We consider the finite element approximation of the Oldroyd-B system of equations, which models a dilute polymeric fluid, in a bounded domain $\mathcal{D} \subset \mathbb{R}^{d}, d=2$ or 3 , subject to no flow boundary conditions. Our schemes are based on approximating the pressure and the symmetric conformation tensor by either (a) piecewise constants or (b) continuous piecewise linears. In case (a) the velocity field is approximated by continuous piecewise quadratics or a reduced version, where the tangential component on each simplicial edge $(d=2)$ or face $(d=3)$ is linear. In case $(\mathrm{b})$ the velocity field is approximated by continuous piecewise quadratics or the mini-element. We show that both of these types of schemes satisfy a free energy bound, which involves the logarithm of the conformation tensor, without any constraint on the time step for the backward Euler type time discretization. This extends the results of Boyaval et al ${ }^{[\mathrm{BLM} 09}$ on this free energy bound. There a piecewise constant approximation of the conformation tensor was necessary to treat the advection term in the stress equation, and a restriction on the time step, based on the initial data, was required to ensure that the approximation to the conformation tensor remained positive definite. Furthermore, for our approximation (b) in the presence of an additional dissipative term in the stress equation and a cut-off on the conformation tensor on certain terms in the system, similar to those

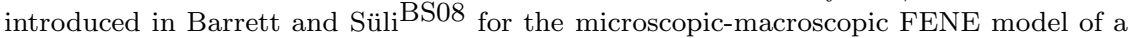
dilute polymeric fluid, we show (subsequence) convergence, as the spatial and temporal discretization parameters tend to zero, towards global-in-time weak solutions of this regularized Oldroyd-B system. Hence, we prove existence of global-in-time weak solutions to this regularized model. Moreover, in the case $d=2$ we carry out this convergence in the absence of cut-offs, but with a time step restriction dependent on the spatial discretization parameter, and hence show existence of a global-in-time weak solution to the Oldroyd-B system with an additional dissipative term in the stress equation.
\end{abstract}

Keywords: Oldroyd-B model, entropy, finite element method, convergence analysis, existence of weak solutions.

AMS Subject Classification: 35Q30, 65M12, 65M60, 76A10, 76M10, 82D60 


\section{Introduction}

\subsection{The standard Oldroyd-B model}

We consider the Oldroyd-B model for a dilute polymeric fluid. The fluid, confined to an open bounded domain $\mathcal{D} \subset \mathbb{R}^{d}(d=2$ or 3$)$ with a Lipschitz boundary $\partial \mathcal{D}$, is governed by the following non-dimensionalized system:

(P) Find $\boldsymbol{u}:(t, \boldsymbol{x}) \in[0, T) \times \mathcal{D} \mapsto \boldsymbol{u}(t, \boldsymbol{x}) \in \mathbb{R}^{d}, p:(t, \boldsymbol{x}) \in \mathcal{D}_{T}:=(0, T) \times \mathcal{D} \mapsto$ $p(t, \boldsymbol{x}) \in \mathbb{R}$ and $\boldsymbol{\sigma}:(t, \boldsymbol{x}) \in[0, T) \times \mathcal{D} \mapsto \boldsymbol{\sigma}(t, \boldsymbol{x}) \in \mathbb{R}_{S}^{d \times d}$ such that

$$
\begin{aligned}
& \operatorname{Re}\left(\frac{\partial \boldsymbol{u}}{\partial t}+(\boldsymbol{u} \cdot \boldsymbol{\nabla}) \boldsymbol{u}\right)=-\boldsymbol{\nabla} p+(1-\varepsilon) \Delta \boldsymbol{u}+\frac{\varepsilon}{\mathrm{Wi}} \operatorname{div} \boldsymbol{\sigma}+\boldsymbol{f} \quad \text { on } \mathcal{D}_{T}, \\
& \begin{array}{ll}
\operatorname{div} \boldsymbol{u}=0 \quad \text { on } \mathcal{D}_{T},
\end{array} \\
& \frac{\partial \boldsymbol{\sigma}}{\partial t}+(\boldsymbol{u} \cdot \boldsymbol{\nabla}) \boldsymbol{\sigma}=(\boldsymbol{\nabla} \boldsymbol{u}) \boldsymbol{\sigma}+\boldsymbol{\sigma}(\boldsymbol{\nabla} \boldsymbol{u})^{T}-\frac{1}{\mathrm{Wi}}(\boldsymbol{\sigma}-\boldsymbol{I}) \quad \text { on } \mathcal{D}_{T}, \\
& \boldsymbol{u}(0, \boldsymbol{x})=\boldsymbol{u}^{0}(\boldsymbol{x}) \quad \forall \boldsymbol{x} \in \mathcal{D}, \\
& \boldsymbol{\sigma}(0, \boldsymbol{x})=\boldsymbol{\sigma}^{0}(\boldsymbol{x}) \quad \forall \boldsymbol{x} \in \mathcal{D}, \\
& \boldsymbol{u}=\mathbf{0} \quad \text { on }(0, T) \times \partial \mathcal{D} .
\end{aligned}
$$

Here $\boldsymbol{u}$ is the velocity of the fluid, $p$ is the hydrostatic pressure, and $\boldsymbol{\sigma}$ is the symmetric conformation tensor of the polymer molecules linked to the symmetric polymeric extra-stress tensor $\boldsymbol{\tau}$ through the relation $\boldsymbol{\sigma}=\boldsymbol{I}+\frac{\mathrm{Wi}}{\varepsilon} \boldsymbol{\tau}$, where $\boldsymbol{I}$ is the $d$-dimensional identity tensor and $\mathbb{R}_{S}^{d \times d}$ denotes symmetric real $d \times d$ matrices. In addition, $\boldsymbol{f}:(t, \boldsymbol{x}) \in \mathcal{D}_{T} \mapsto \boldsymbol{f}(t, \boldsymbol{x}) \in \mathbb{R}^{d}$ is the given density of body forces acting on the fluid; and the following given parameters are dimensionless: the Reynolds number $\operatorname{Re} \in \mathbb{R}_{>0}$, the Weissenberg number Wi $\in \mathbb{R}_{>0}$, and the elastic-to-viscous viscosity fraction $\varepsilon \in(0,1)$. For the sake of simplicity, we will limit ourselves to the no flow boundary condition (1.1f). Finally, $\boldsymbol{\nabla} \boldsymbol{u}(t, \boldsymbol{x}) \in \mathbb{R}^{d \times d}$ with $[\boldsymbol{\nabla} \boldsymbol{u}]_{i j}=\frac{\partial \boldsymbol{u}_{i}}{\partial \boldsymbol{x}_{j}}$, and $(\operatorname{div} \boldsymbol{\sigma})(t, \boldsymbol{x}) \in \mathbb{R}^{d}$ with $[\operatorname{div} \boldsymbol{\sigma}]_{i}=\sum_{j=1}^{d} \frac{\partial \boldsymbol{\sigma}_{i j}}{\partial \boldsymbol{x}_{j}}$.

Unfortunately, at present there is no proof of existence of global-in-time weak solutions to $(\mathrm{P})$ available in the literature. Local-in-time existence results for $(\mathrm{P})$ for sufficiently smooth initial data, and global-in-time existence results for sufficiently small initial data can be found in Guillopé and Saut ${ }^{\text {GS90 }}$ for a Hilbert space framework, and in Fernández-Cara et al. ${ }^{\text {FCGO02 }}$ for a more general Banach space framework. Global-in-time existence results for the corotational version of $(\mathrm{P})$; that is, where $\boldsymbol{\nabla} \boldsymbol{u}$ in (1.1c) is replaced by its anti-symmetric part $\frac{1}{2}\left(\boldsymbol{\nabla} \boldsymbol{u}-(\boldsymbol{\nabla} \boldsymbol{u})^{T}\right)$ can be found in Lions and Masmoudi.LM00 We note that such a simple change to the model leads to a vast simplification mathematically, but, of course, it is not justified on physical grounds. Finally, global-in-time existence results for $(\mathrm{P})$ in the case $\boldsymbol{f} \equiv \mathbf{0}$ and for initial data close to equilibrium can be found in Lei et al. ${ }_{\text {LLZ08 }}$.

This paper considers some finite element approximations of the Oldroyd-B system, possibly with some regularization. In the regularized case, we show (subsequence) convergence of the approximation, as the spatial and temporal discretiza- 
tion parameters tend to zero, and so establish the existence of global-in-time weak solutions of these regularized versions of the Oldroyd-B system. The first of these regularized problems is $\left(\mathrm{P}_{\alpha}\right)$ obtained by adding the dissipative term $\alpha \Delta \sigma$ for a given $\alpha \in \mathbb{R}_{>0}$ to the right-hand side of (1.1c), as considered computationally in Sureshkumar and Beris,, SB95 with an additional no flux boundary condition for $\sigma$ on $\partial \mathcal{D}$. The second is $\left(\mathrm{P}_{\alpha}^{L}\right)$ where, in addition to the regularization in $\left(\mathrm{P}_{\alpha}\right)$, the conformation tensor $\boldsymbol{\sigma}$ is replaced by the cut-off $\beta^{L}(\boldsymbol{\sigma})$ on the right-hand side of (1.1a) and in the terms involving $\boldsymbol{u}$ in (1.1c), where $\beta^{L}(s):=\min \{s, L\}$ for a given $L \gg 1$. Similar regularizations have been introduced for the microscopicmacroscopic dumbbell model of dilute polymers with a finitely extensible nonlinear elastic (FENE) spring law, see Barrett and Süli BS08 and for the convergence of the finite element approximation of such models, see Barrett and Süli.BS10 In fact, it is argued in Barrett and Süli $\frac{\mathrm{BS} 07}{}$ and Schieber $\underline{\operatorname{Sch} 06}$ that the dissipative term $\alpha \Delta \sigma$ is not a regularization, but is present in the original model with a positive $\alpha \ll 1$. Here we recall that the Oldroyd-B system is the macroscopic closure of the microscopic-macroscopic dumbbell model with a Hookean spring law, see e.g. Barrett and Süli. $\mathrm{BS07}$

Overall the aims of this paper are threefold. First, we extend previous results in Boyaval et al. ${ }^{\text {BLM09 }}$ for a finite element approximation of $(\mathrm{P})$ using essentially the backward Euler scheme in time and based on approximating the pressure and the symmetric conformation tensor by piecewise constants; and the velocity field with continuous piecewise quadratics or a reduced version, where the tangential component on each simplicial edge $(d=2)$ or face $(d=3)$ is linear. We show that solutions of this numerical scheme satisfy a discrete free energy bound, which involves the logarithm of the conformation tensor, without any constraint on the time step, whereas a time constraint based on the initial data was required in Boyaval et al. BLM09 in order to ensure that the approximation to the conformation tensor $\sigma$ remained positive definite. See also Lee and Xu, $\underline{\mathrm{LX} 06}$ where the difficulties of maintaining the positive definiteness of approximations to $\sigma$ are also discussed. We achieve our result by first introducing problem $\left(\mathrm{P}_{\delta}\right)$, based on a regularization parameter $\delta \in \mathbb{R}_{>0}$. $\left(\mathrm{P}_{\delta}\right)$ satisfies a regularized free energy estimate based on a regularization of $\ln$ and is valid without the positive definiteness constraint on the deformation tensor.

Second, we show that it is possible to approximate $(\mathrm{P})$ with a continuous (piecewise linear) approximation of the conformation tensor, such that a discrete free energy bound still holds. We note that a piecewise constant approximation of the conformation tensor was necessary in Boyaval et al BLM09 in order to treat the advection term in (1.1c) and still obtain a discrete free energy bound.

Third, we show (subsequence) convergence, as the spatial and temporal discretization parameters tend to zero, of this latter approximation in the presence of the regularization terms stated above to global-in-time weak solutions of the corresponding regularized form of $(\mathrm{P})$.

The outline of this paper is as follows. We end Section 1 by introducing our 
notation and auxiliary results. In Section 2 we introduce our regularizations of $\ln$ based on the parameter $\delta \in\left(0, \frac{1}{2}\right]$ and the cut-off $L \geq 2$. We introduce our regularized problem $\left(\mathrm{P}_{\delta}\right)$, and show a formal free energy estimate for it. In Section 3 , on assuming that $\mathcal{D}$ is a polytope, we introduce our finite element approximation of $\left(\mathrm{P}_{\delta}\right),\left(\mathrm{P}_{\delta, h}^{\Delta t}\right)$ based on approximating the pressure and the symmetric conformation tensor by piecewise constants; and the velocity field with continuous piecewise quadratics or a reduced version, where the tangential component on each simplicial edge $(d=2)$ or face $(d=3)$ is linear. Using the Brouwer fixed point theorem, we prove existence of a solution to $\left(\mathrm{P}_{\delta, h}^{\Delta t}\right)$ and show that it satisfies a discrete regularized free energy estimate for any choice of time step; see Theorem 3.1. We conclude by showing that, in the limit $\delta \rightarrow 0_{+}$, these solutions of $\left(\mathrm{P}_{\delta, h}^{\Delta t}\right)$ converge to a solution of $\left(\mathrm{P}_{h}^{\Delta t}\right)$ with the approximation of the conformation tensor being positive definite. Moreover, this solution of $\left(\mathrm{P}_{h}^{\Delta t}\right)$ satisfies a discrete free energy estimate; see Theorem 3.2 ,

In Section 4 we introduce our regularizations $\left(\mathrm{P}_{\alpha}^{(L)}\right)$ of $(\mathrm{P})$ involving the dissipative term $\alpha \Delta \boldsymbol{\sigma}$ on the right-hand side of (1.1c), and possibly the cut-off $\beta^{L}(\boldsymbol{\sigma})$ on certain terms involving $\sigma$ in (1.1a c). We then introduce the corresponding regularized version $\left(\mathrm{P}_{\alpha, \delta}^{(L)}\right)$, and show a formal free energy estimate for it. In Section 5 we introduce our finite element approximation of $\left(\mathrm{P}_{\alpha, \delta}^{(L)}\right),\left(\mathrm{P}_{\alpha, \delta, h}^{(L,) \Delta t}\right)$ based on approximating the pressure and the symmetric conformation tensor by continuous piecewise linears; and the velocity field with continuous piecewise quadratics or the mini-element. Here we assume that the finite element mesh consists of non-obtuse simplices. Using the Brouwer fixed point theorem, we prove existence of a solution to $\left(\mathrm{P}_{\alpha, \delta, h}^{(L,) \Delta t}\right)$ and show that is satisfies a discrete regularized free energy estimate for any choice of time step; see Theorem [5.1. We conclude by showing that, in the limit $\delta \rightarrow 0_{+}$, these solutions of $\left(\mathrm{P}_{\alpha, \delta, h}^{(L,) \Delta t}\right)$ converge to a solution of $\left(\mathrm{P}_{\alpha, h}^{(L,) \Delta t}\right)$ with the approximation of the conformation tensor being positive definite. Moreover, this solution of $\left(\mathrm{P}_{\alpha, h}^{(L,) \Delta t}\right)$ satisfies a discrete free energy estimate; see Theorem 5.2

In Section [6] we assume, in addition, that $\mathcal{D}$ is a convex polytope and that the finite element mesh consists of quasi-uniform simplices. We then prove (subsequence) convergence of the solutions of $\left(\mathrm{P}_{\alpha, h}^{L, \Delta t}\right)$, as the spatial and temporal discretization parameters tend to zero, to global-in-time weak solutions of $\left(\mathrm{P}_{\alpha}^{L}\right)$; see Theorem 6.2. Finally in Section 7 , on further assuming that $d=2$ and a time step restriction dependent on the spatial discretization parameter, we prove (subsequence) convergence of the solutions of $\left(\mathrm{P}_{\alpha, h}^{\Delta t}\right)$, as the spatial and temporal discretization parameters tend to zero, to global-in-time weak solutions of $\left(\mathrm{P}_{\alpha}\right)$; see Theorem 7.2. We note that these existence results for $\left(\mathrm{P}_{\alpha}^{(L)}\right)$ are new to the literature. In addition, the corresponding $L^{\infty}\left(0, T ; L^{2}(\Omega)\right) \cap L^{2}\left(0, T ; H^{1}(\Omega)\right)$ norms of the velocity solution $\boldsymbol{u}_{\alpha}^{(L)}$ of $\left(\mathrm{P}_{\alpha}^{(L)}\right)$ are independent of the regularization parameters $\alpha$ (and $L$ ).

In a forthcoming paper $\frac{\mathrm{BB} 09}{}$ we will extend the ideas in this paper to a related

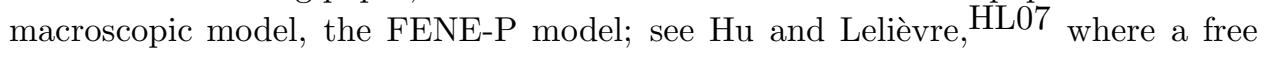
energy estimate is developed for such a model, as well as Oldroyd-B. In addition, 
we will report in the near future on numerical computations based on the finite element approximations in this paper and Barrett and Boyaval.BB09

\subsection{Notation and auxiliary results}

The absolute value and the negative part of a real number $s \in \mathbb{R}$ are denoted by $|s|:=\max \{s,-s\}$ and $[s]_{-}=\min \{s, 0\}$, respectively. In addition to $\mathbb{R}_{S}^{d \times d}$, the set of symmetric $\mathbb{R}^{d \times d}$ matrices, we let $\mathbb{R}_{S P D}^{d \times d}$ be the set of symmetric positive definite $\mathbb{R}^{d \times d}$ matrices. We adopt the following notation for inner products:

$$
\begin{array}{cc}
\boldsymbol{v} \cdot \boldsymbol{w}:=\sum_{i=1}^{d} \boldsymbol{v}_{i} \boldsymbol{w}_{i} \equiv \boldsymbol{v}^{T} \boldsymbol{w}=\boldsymbol{w}^{T} \boldsymbol{v} & \forall \boldsymbol{v}, \boldsymbol{w} \in \mathbb{R}^{d}, \\
\boldsymbol{\phi}: \boldsymbol{\psi}:=\sum_{i=1}^{d} \sum_{j=1}^{d} \boldsymbol{\phi}_{i j} \boldsymbol{\psi}_{i j} \equiv \operatorname{tr}\left(\boldsymbol{\phi}^{T} \boldsymbol{\psi}\right)=\operatorname{tr}\left(\boldsymbol{\psi}^{T} \boldsymbol{\phi}\right) & \forall \boldsymbol{\phi}, \boldsymbol{\psi} \in \mathbb{R}^{d \times d}, \\
\boldsymbol{\nabla} \boldsymbol{\phi}:: \boldsymbol{\nabla} \boldsymbol{\psi}:=\sum_{i=1}^{d} \sum_{j=1}^{d} \boldsymbol{\nabla} \boldsymbol{\phi}_{i j} \cdot \boldsymbol{\nabla} \boldsymbol{\psi}_{i j} & \forall \boldsymbol{\phi}, \boldsymbol{\psi} \in \mathbb{R}^{d \times d}
\end{array}
$$

where ${ }^{T}$ and $\operatorname{tr}(\cdot)$ denote transposition and trace, respectively. The corresponding norms are

$$
\begin{aligned}
\|\boldsymbol{v}\|:=(\boldsymbol{v} \cdot \boldsymbol{v})^{\frac{1}{2}}, & \|\boldsymbol{\nabla} \boldsymbol{v}\|:=(\boldsymbol{\nabla} \boldsymbol{v}: \boldsymbol{\nabla} \boldsymbol{v})^{\frac{1}{2}} & & \forall \boldsymbol{v} \in \mathbb{R}^{d} ; \\
\|\boldsymbol{\phi}\|:=(\boldsymbol{\phi}: \boldsymbol{\phi})^{\frac{1}{2}}, & \|\boldsymbol{\nabla} \boldsymbol{\phi}\|:=(\boldsymbol{\nabla} \boldsymbol{\phi}:: \nabla \boldsymbol{\nabla})^{\frac{1}{2}}, & & \forall \boldsymbol{\phi} \in \mathbb{R}^{d \times d} .
\end{aligned}
$$

We will use on several occasions that $\operatorname{tr}(\phi)=\operatorname{tr}\left(\phi^{T}\right)$ and $\operatorname{tr}(\phi \psi)=\operatorname{tr}(\boldsymbol{\phi} \phi)$ for all $\boldsymbol{\phi}, \boldsymbol{\psi} \in \mathbb{R}^{d \times d}$. In particular, we note that:

$$
\begin{array}{rlrl}
\phi \chi^{T}: \psi & =\chi \phi: \psi=\chi: \psi \phi & & \forall \phi, \psi \in \mathbb{R}_{S}^{d \times d}, \chi \in \mathbb{R}^{d \times d}, \\
\|\psi \phi\| & \leq\|\psi\|\|\phi\| & \forall \phi, \psi \in \mathbb{R}^{d \times d} .
\end{array}
$$

In addition, for any $\phi \in \mathbb{R}_{S}^{d \times d}$, there exists a diagonal decomposition

$$
\phi=\mathbf{O}^{T} \mathbf{D O} \quad \Rightarrow \quad \operatorname{tr}(\phi)=\operatorname{tr}(\mathbf{D}),
$$

where $\mathbf{O} \in \mathbb{R}^{d \times d}$ is an orthogonal matrix and $\mathbf{D} \in \mathbb{R}^{d \times d}$ a diagonal matrix. Hence, for any $g: \mathbb{R} \rightarrow \mathbb{R}$, one can define $g(\phi) \in \mathbb{R}_{S}^{d \times d}$ as

$$
g(\boldsymbol{\phi}):=\mathbf{O}^{T} g(\mathbf{D}) \mathbf{O} \quad \Rightarrow \quad \operatorname{tr}(g(\boldsymbol{\phi}))=\operatorname{tr}(g(\mathbf{D})),
$$

where $g(\mathbf{D}) \in \mathbb{R}_{S}^{d \times d}$ is the diagonal matrix with entries $[g(\mathbf{D})]_{i i}=g\left(\mathbf{D}_{i i}\right), i=1 \rightarrow d$. Although the diagonal decomposition (1.5) is not unique, (1.6) uniquely defines $g(\phi)$. Similarly, one can define $g(\phi) \in \mathbb{R}_{S P D}^{d \times d}$, when $\phi \in \mathbb{R}_{S P D}^{d \times d}$ and $g: \mathbb{R}_{>0} \rightarrow \mathbb{R}$. We note for later purposes that the choice $g(s)=|s|$ for $s \in \mathbb{R}$ yields that

$$
d^{-1}(\operatorname{tr}(|\phi|))^{2} \leq\|\phi\|^{2} \leq(\operatorname{tr}(|\phi|))^{2} \quad \forall \phi \in \mathbb{R}_{S}^{d \times d} .
$$

We adopt the standard notation for Sobolev spaces, e.g. $H^{1}(\mathcal{D}):=\{\eta: \mathcal{D} \rightarrow$ $\left.\mathbb{R}: \int_{\mathcal{D}}\left[|\eta|^{2}+\|\nabla \eta\|^{2}\right]<\infty\right\}$ with $H_{0}^{1}(\mathcal{D})$ being the closure of $C_{0}^{\infty}(\mathcal{D})$ for the 
corresponding norm $\|\cdot\|_{H^{1}(\mathcal{D})}$. We denote the associated semi-norm as $|\cdot|_{H^{1}(\mathcal{D})}$. The topological dual of the Hilbert space $H_{0}^{1}(\mathcal{D})$, with pivot space $L^{2}(\mathcal{D})$, will be denoted by $H^{-1}(\mathcal{D})$. We denote the duality pairing between $H^{-1}(\mathcal{D})$ and $H_{0}^{1}(\mathcal{D})$ as $\langle\cdot, \cdot\rangle_{H_{0}^{1}(\mathcal{D})}$. Such function spaces are naturally extended when the range $\mathbb{R}$ is replaced by $\mathbb{R}^{d}, \mathbb{R}^{d \times d}$ and $\mathbb{R}_{S}^{d \times d}$; e.g. $H^{1}(\mathcal{D})$ becomes $\left[H^{1}(\mathcal{D})\right]^{d},\left[H^{1}(\mathcal{D})\right]^{d \times d}$ and $\left[H^{1}(\mathcal{D})\right]_{S}^{d \times d}$, respectively. For ease of notation, we write the corresponding norms and semi-norms as $\|\cdot\|_{H^{1}(\mathcal{D})}$ and $|\cdot|_{H^{1}(\mathcal{D})}$, respectively, as opposed to e.g. $\|\cdot\|_{\left[H^{1}(\mathcal{D})\right]^{d}}$ and $|\cdot|_{\left[H^{1}(\mathcal{D})\right]^{d}}$, respectively. Similarly, we write $\langle\cdot, \cdot\rangle_{H_{0}^{1}(\mathcal{D})}$ for the duality pairing between e.g. $\left[H^{-1}(\mathcal{D})\right]^{d}$ and $\left[H_{0}^{1}(\mathcal{D})\right]^{d}$. We recall the Poincaré inequality

$$
\int_{\mathcal{D}}\|\boldsymbol{v}\|^{2} \leq C_{P} \int_{\mathcal{D}}\|\boldsymbol{\nabla} \boldsymbol{v}\|^{2} \quad \forall \boldsymbol{v} \in\left[H_{0}^{1}(D)\right]^{d},
$$

where $C_{P} \in \mathbb{R}_{>0}$ depends only on $\mathcal{D}$. For notational convenience, we introduce also convex sets such as $\left[H^{1}(\mathcal{D})\right]_{S P D}^{d \times d}:=\left\{\phi \in\left[H^{1}(\mathcal{D})\right]_{S}^{d \times d}: \phi \in \mathbb{R}_{S P D}^{d \times d}\right.$ a.e. in $\left.\mathcal{D}\right\}$. Moreover, in order to analyse $(\mathrm{P})$, we adopt the notation

$$
\begin{aligned}
& \mathrm{W}:=\left[H_{0}^{1}(\mathcal{D})\right]^{d}, \quad \mathrm{Q}:=L^{2}(\mathcal{D}), \quad \mathrm{V}:=\left\{\boldsymbol{v} \in \mathrm{W}: \int_{\mathcal{D}} q \operatorname{div} \boldsymbol{v}=0 \quad \forall q \in \mathrm{Q}\right\}, \\
& \mathrm{S}:=\left[L^{1}(\mathcal{D})\right]_{S}^{d \times d} \quad \text { and } \quad \mathrm{S}_{P D}:=\left[L^{1}(\mathcal{D})\right]_{S P D}^{d \times d} .
\end{aligned}
$$

Finally, throughout the paper $C$ will denote a generic positive constant independent of the regularization parameters $\delta, L$ and $\alpha$; and the mesh parameters $h$ and $\Delta t$.

\section{Formal free energy estimates for a regularized problem $\left(\mathbf{P}_{\delta}\right)$}

\subsection{Some regularizations}

Let $G: s \in \mathbb{R}_{>0} \mapsto \ln s \in \mathbb{R}$ be the logarithm function, whose domain of definition can be straightforwardly extended to the set of symmetric positive definite matrices using (1.5). We define the following two concave $C^{1}(\mathbb{R})$ regularizations of $G$ based on given parameters $L>1>\delta>0$ :

$$
\begin{aligned}
& G_{\delta}: s \in \mathbb{R} \mapsto\left\{\begin{array}{ll}
G(s), & \forall s \geq \delta \\
\frac{s}{\delta}+G(\delta)-1, & \forall s \leq \delta
\end{array} \text { and } G_{\delta}^{L}: s \in \mathbb{R} \mapsto\left\{\begin{array}{ll}
G^{L}(s), & \forall s \geq \delta \\
G_{\delta}(s), & \forall s \leq \delta
\end{array},\right.\right. \\
& \text { where } G^{L}: s \in \mathbb{R}_{>0} \mapsto\left\{\begin{array}{ll}
\frac{s}{L}+G(L)-1, & \forall s \geq L \\
G(s), & \forall s \in(0, L]
\end{array} .\right.
\end{aligned}
$$

We define also the following scalar functions

$$
\beta_{\delta}^{(L)}(s):=\left(G_{\delta}^{(L)^{\prime}}(s)\right)^{-1} \quad \forall s \in \mathbb{R} \quad \text { and } \quad \beta^{(L)}(s):=\left(G^{(L)^{\prime}}(s)\right)^{-1} \quad \forall s \in \mathbb{R}_{>0} ;
$$

where, here and throughout this paper, ${ }^{(\star)}$ denotes an expression with or without the superscript $\star$, and a similar convention with subscripts. Hence we have that

$$
\beta_{\delta}: s \in \mathbb{R} \mapsto \max \{s, \delta\}, \quad \beta_{\delta}^{L}: s \in \mathbb{R} \mapsto \min \left\{\beta_{\delta}(s), L\right\},
$$




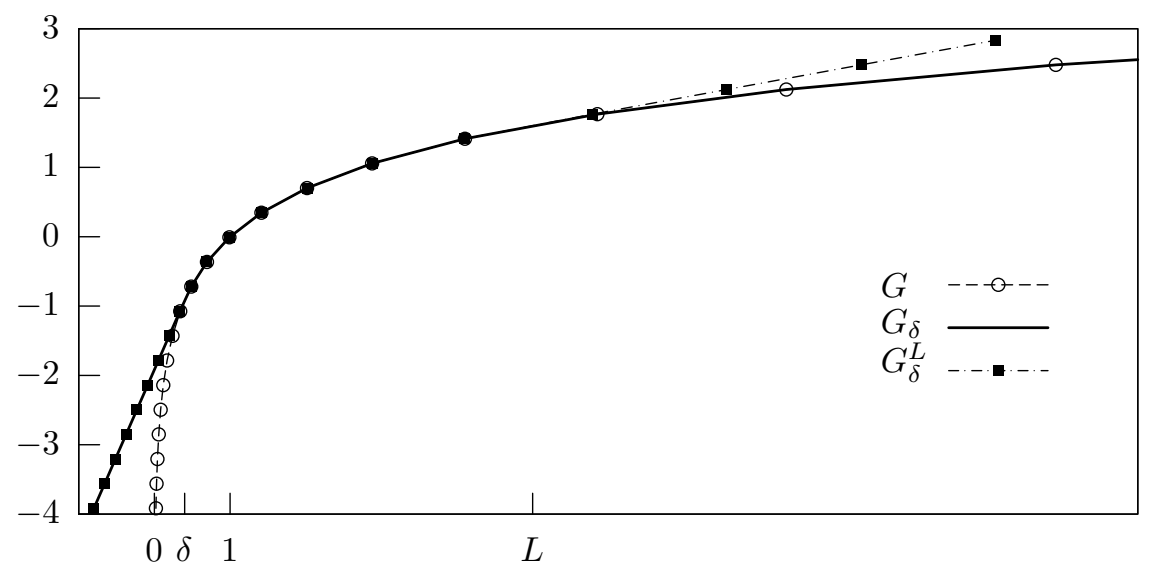

Figure 1. The function $G$ and its regularizations.

$$
\beta: s \in \mathbb{R}_{>0} \mapsto s \quad \text { and } \quad \beta^{L}: s \in \mathbb{R}_{>0} \mapsto \min \{\beta(s), L\} .
$$

We note for example that

$$
\left\|\beta_{\delta}^{L}(\phi)\right\|^{2} \leq d L^{2} \quad \forall \phi \in \mathbb{R}_{S}^{d \times d} \quad \text { and } \quad\left\|\beta^{L}(\phi)\right\|^{2} \leq d L^{2} \quad \forall \phi \in \mathbb{R}_{S P D}^{d \times d} .
$$

Introducing the concave $C^{1}(\mathbb{R})$ functions

$$
H_{\delta}^{L}(s):=G_{L^{-1}}^{\delta^{-1}}(s) \quad \forall s \in \mathbb{R} \quad \text { and } \quad H_{\delta}(s):=G^{\delta^{-1}}(s) \quad \forall s \in \mathbb{R}_{>0},
$$

it follows from (2.1) and (2.3) that

$$
H_{\delta}^{(L)^{\prime}}\left(G_{\delta}^{(L)^{\prime}}(s)\right)=\beta_{\delta}^{(L)}(s) \quad \forall s \in \mathbb{R} .
$$

For later purposes, we prove the following results concerning these functions.

Lemma 2.1. The following hold for any $\phi, \psi \in \mathbb{R}_{S}^{d \times d}$ and for any $L>1>\delta>0$ that

$$
\begin{aligned}
{\left[\beta_{\delta}^{(L)}(\phi)\right]\left[G_{\delta}^{(L)^{\prime}}(\boldsymbol{\phi})\right] } & =\left[G_{\delta}^{(L)^{\prime}}(\boldsymbol{\phi})\right]\left[\beta_{\delta}^{(L)}(\boldsymbol{\phi})\right]=\boldsymbol{I}, \\
\operatorname{tr}\left(\beta_{\delta}^{(L)}(\boldsymbol{\phi})+\left[\beta_{\delta}^{(L)}(\boldsymbol{\phi})\right]^{-1}-2 \boldsymbol{I}\right) & \geq 0, \\
\operatorname{tr}\left(\boldsymbol{\phi}-G_{\delta}^{(L)}(\boldsymbol{\phi})-\boldsymbol{I}\right) & \geq 0, \\
\left(\boldsymbol{\phi}-\beta_{\delta}^{(L)}(\boldsymbol{\phi})\right):\left(\boldsymbol{I}-G_{\delta}^{(L)^{\prime}}(\boldsymbol{\phi})\right) & \geq 0, \\
(\boldsymbol{\phi}-\boldsymbol{\psi}):\left(G_{\delta}^{(L)^{\prime}}(\boldsymbol{\psi})\right) & \geq \operatorname{tr}\left(G_{\delta}^{(L)}(\boldsymbol{\phi})-G_{\delta}^{(L)}(\boldsymbol{\psi})\right), \\
-(\boldsymbol{\phi}-\boldsymbol{\psi}):\left(G_{\delta}^{(L)^{\prime}}(\boldsymbol{\phi})-G_{\delta}^{(L)^{\prime}}(\boldsymbol{\psi})\right) & \geq \delta^{2}\left\|G_{\delta}^{(L)^{\prime}}(\boldsymbol{\phi})-G_{\delta}^{(L)^{\prime}}(\boldsymbol{\psi})\right\|^{2} .
\end{aligned}
$$


In addition, if $\delta \in\left(0, \frac{1}{2}\right]$ and $L \geq 2$ we have that

$$
\operatorname{tr}\left(\phi-G_{\delta}^{(L)}(\phi)\right) \geq\left\{\begin{array}{l}
\frac{1}{2}\|\phi\| \\
\frac{1}{2 \delta}\left\|[\phi]_{-}\right\|
\end{array} \quad \text { and } \quad \phi:\left(I-G_{\delta}^{(L)^{\prime}}(\phi)\right) \geq \frac{1}{2}\|\phi\|-d .\right.
$$

Proof. The result (2.7a) follows immediately from (1.6) and as $\beta_{\delta}^{(L)}(s)=G_{\delta}^{(L)^{\prime}}(s)$ for all $s \in \mathbb{R}$. The desired results (2.7b-d) follow similarly, on noting the scalar inequalities $\beta_{\delta}^{(L)}(s)+\left[\beta_{\delta}^{(L)}(s)\right]^{-1} \geq 2, s-G_{\delta}^{(L)}(s) \geq 1$ and $\left(s-\beta_{\delta}^{(L)}(s)\right)\left(1-G_{\delta}^{(L)^{\prime}}(s)\right) \geq$ 0 for all $s \in \mathbb{R}$.

We note that $G_{\delta}^{(L)}$ are concave functions like $G$, and hence they satisfy the following inequality

$$
\left(s_{1}-s_{2}\right) G_{\delta}^{(L)^{\prime}}\left(s_{2}\right) \geq G_{\delta}^{(L)}\left(s_{1}\right)-G_{\delta}^{(L)}\left(s_{2}\right) \quad \forall s_{1}, s_{2} \in \mathbb{R} ;
$$

Hence for any $\boldsymbol{\phi}, \boldsymbol{\psi} \in \mathbb{R}_{S}^{d \times d}$ with $\boldsymbol{\phi}=\mathbf{O}_{\phi}^{T} \mathbf{D}_{\phi} \mathbf{O}_{\phi}$ and $\boldsymbol{\psi}=\mathbf{O}_{\psi}^{T} \mathbf{D}_{\psi} \mathbf{O}_{\psi}$, where $\mathbf{O}_{\phi}, \mathbf{O}_{\psi} \in \mathbb{R}^{d \times d}$ orthogonal and $\mathbf{D}_{\phi}, \mathbf{D}_{\psi} \in \mathbb{R}^{d \times d}$ diagonal, we have, on noting the properties of trace, that

$$
(\phi-\psi): G_{\delta}^{(L)^{\prime}}(\boldsymbol{\psi})=\operatorname{tr}\left((\boldsymbol{\phi}-\boldsymbol{\psi}) G_{\delta}^{(L)^{\prime}}(\boldsymbol{\psi})\right)=\operatorname{tr}\left(\left(\mathbf{O}^{T} \mathbf{D}_{\phi} \mathbf{O}-\mathbf{D}_{\psi}\right) G_{\delta}^{(L)^{\prime}}\left(\mathbf{D}_{\psi}\right)\right),
$$

where $\mathbf{O}=\mathbf{O}_{\phi} \mathbf{O}_{\psi}^{T} \in \mathbb{R}^{d \times d}$ is orthogonal and hence $\sum_{i=1}^{d}\left[\mathbf{O}_{i j}\right]^{2}=\sum_{i=1}^{d}\left[\mathbf{O}_{j i}\right]^{2}=1$ for $j=1 \rightarrow d$. Therefore we have, on noting these properties of $\mathbf{O}$, (2.9) and (1.6), that

$$
\begin{aligned}
\operatorname{tr}\left(\left(\mathbf{O}^{T} \mathbf{D}_{\phi} \mathbf{O}-\mathbf{D}_{\psi}\right) G_{\delta}^{(L)^{\prime}}\left(\mathbf{D}_{\psi}\right)\right) & =\sum_{i=1}^{d}\left(\sum_{j=1}^{d}\left[\mathbf{O}_{j i}\right]^{2}\left[\mathbf{D}_{\phi}\right]_{j j}-\left[\mathbf{D}_{\psi}\right]_{i i}\right)\left[G_{\delta}^{(L)^{\prime}}\left(\mathbf{D}_{\psi}\right)\right]_{i i} \\
& =\sum_{i=1}^{d} \sum_{j=1}^{d}\left[\mathbf{O}_{j i}\right]^{2}\left(\left[\mathbf{D}_{\phi}\right]_{j j}-\left[\mathbf{D}_{\psi}\right]_{i i}\right)\left[G_{\delta}^{(L)^{\prime}}\left(\mathbf{D}_{\psi}\right)\right]_{i i} \\
& \geq \sum_{i=1}^{d} \sum_{j=1}^{d}\left[\mathbf{O}_{j i}\right]^{2}\left(\left[G_{\delta}^{(L)}\left(\mathbf{D}_{\phi}\right)\right]_{j j}-\left[G_{\delta}^{(L)}\left(\mathbf{D}_{\psi}\right)\right]_{i i}\right) \\
& =\operatorname{tr}\left(G_{\delta}^{(L)}\left(\mathbf{D}_{\phi}\right)\right)-\operatorname{tr}\left(G_{\delta}^{(L)}\left(\mathbf{D}_{\psi}\right)\right) \\
& =\operatorname{tr}\left(G_{\delta}^{(L)}(\phi)-G_{\delta}^{(L)}(\boldsymbol{\psi})\right) .
\end{aligned}
$$

Combining (2.10) and (2.11) yields the desired result (2.7e).

We note that $-G_{\delta}^{(L)^{\prime}} \in C^{0,1}(\mathbb{R})$ is monotonically increasing with Lipschitz constant $\delta^{-2}$ and so

$$
-\left(s_{1}-s_{2}\right)\left(G_{\delta}^{(L)^{\prime}}\left(s_{1}\right)-G_{\delta}^{(L)^{\prime}}\left(s_{2}\right)\right) \geq \delta^{2}\left[G_{\delta}^{(L)^{\prime}}\left(s_{1}\right)-G_{\delta}^{(L)^{\prime}}\left(s_{2}\right)\right]^{2} \quad \forall s_{1}, s_{2} \in \mathbb{R} .
$$

Then, similarly to (2.10) and (2.11), we have, on noting (2.12), that

$$
-(\phi-\psi):\left(G_{\delta}^{(L)^{\prime}}(\phi)-G_{\delta}^{(L)^{\prime}}(\boldsymbol{\psi})\right)
$$




$$
\begin{aligned}
& =-\left[\operatorname{tr}\left(\left(\mathbf{D}_{\phi}-\mathbf{O} \mathbf{D}_{\psi} \mathbf{O}^{T}\right) G_{\delta}^{(L)^{\prime}}\left(\mathbf{D}_{\phi}\right)\right)-\operatorname{tr}\left(\left(\mathbf{O}^{T} \mathbf{D}_{\phi} \mathbf{O}-\mathbf{D}_{\psi}\right) G_{\delta}^{(L)^{\prime}}\left(\mathbf{D}_{\psi}\right)\right)\right] \\
& =-\sum_{i=1}^{d} \sum_{j=1}^{d}\left[\mathbf{O}_{j i}\right]^{2}\left(\left[\mathbf{D}_{\phi}\right]_{j j}-\left[\mathbf{D}_{\psi}\right]_{i i}\right)\left(\left[G_{\delta}^{(L)^{\prime}}\left(\mathbf{D}_{\phi}\right)\right]_{j j}-\left[G_{\delta}^{(L)^{\prime}}\left(\mathbf{D}_{\psi}\right)\right]_{i i}\right) \\
& \geq \delta^{2} \sum_{i=1}^{d} \sum_{j=1}^{d}\left[\mathbf{O}_{j i}\right]^{2}\left(\left[G_{\delta}^{(L)^{\prime}}\left(\mathbf{D}_{\phi}\right)\right]_{j j}-\left[G_{\delta}^{(L)^{\prime}}\left(\mathbf{D}_{\psi}\right)\right]_{i i}\right)^{2} \\
& =\delta^{2} \operatorname{tr}\left(\left(G_{\delta}^{(L)^{\prime}}(\boldsymbol{\phi})-G_{\delta}^{(L)^{\prime}}(\boldsymbol{\psi})\right)^{2}\right)=\delta^{2}\left\|G_{\delta}^{(L)^{\prime}}(\boldsymbol{\phi})-G_{\delta}^{(L)^{\prime}}(\boldsymbol{\psi})\right\|^{2}
\end{aligned}
$$

and hence the desired result (2.7f).

Finally the results (2.8) follow from (1.6) and (1.7) on noting the following scalar inequalities

$$
s-G_{\delta}^{(L)}(s) \geq\left\{\begin{array}{l}
\frac{1}{2}|s| \\
\frac{1}{2 \delta}\left|[s]_{-}\right|
\end{array} \quad \text { and } \quad s\left(1-G_{\delta}^{(L)^{\prime}}(s)\right) \geq \frac{1}{2}|s|-1 \quad \forall s \in \mathbb{R}\right.
$$

which are easily deduced if $\delta \in\left(0, \frac{1}{2}\right]$ and $L \geq 2$.

Clearly (2.7e) holds for any concave function $g \in C^{1}(\mathbb{R})$, not just $G_{\delta}^{(L)}$, and this implies that

$$
(\boldsymbol{\phi}-\boldsymbol{\psi}): g^{\prime}(\boldsymbol{\psi}) \geq \operatorname{tr}(g(\boldsymbol{\phi})-g(\boldsymbol{\psi})) \geq(\boldsymbol{\phi}-\boldsymbol{\psi}): g^{\prime}(\boldsymbol{\phi}) \quad \forall \boldsymbol{\phi}, \boldsymbol{\psi} \in \mathbb{R}_{S}^{d \times d} .
$$

For a convex function $g \in C^{1}(\mathbb{R})$, the inequalities in (2.15) are reversed. Hence for any concave or convex function $g \in C^{1}(\mathbb{R})$ and for any $\phi \in C^{1}\left([0, T) ; \mathbb{R}_{S}^{d \times d}\right)$ one can deduce from the above that

$$
\frac{d}{d t} \operatorname{tr}(g(\phi))=\operatorname{tr}\left(\frac{d \phi}{d t} g^{\prime}(\phi)\right)=\left(\frac{d \phi}{d t}\right): g^{\prime}(\phi) \quad \forall t \in[0, T) .
$$

Of course, a similar result holds true for spatial derivatives. Furthermore, these results hold true if $\phi$ is in addition positive definite, and $g \in C^{1}\left(\mathbb{R}_{>0}\right)$ is a concave or convex function. Finally, we note that one can use the approach in (2.11) to show that if $g \in C^{0,1}(\mathbb{R})$ with Lipschitz constant $g_{\text {Lip }}$, then

$$
\|g(\boldsymbol{\phi})-g(\boldsymbol{\psi})\| \leq g_{\text {Lip }}\|\boldsymbol{\phi}-\boldsymbol{\psi}\| \quad \forall \boldsymbol{\phi}, \boldsymbol{\psi} \in \mathbb{R}_{S}^{d \times d} .
$$

\subsection{Regularized problem $\left(P_{\delta}\right)$}

Using the regularizations $G_{\delta}$ introduced above with parameter $\delta$ we consider the following regularization of $(\mathrm{P})$ for a given $\delta \in\left(0, \frac{1}{2}\right]$ :

$\left(\mathbf{P}_{\delta}\right)$ Find $\boldsymbol{u}_{\delta}:(t, \boldsymbol{x}) \in[0, T) \times \mathcal{D} \mapsto \boldsymbol{u}_{\delta}(t, \boldsymbol{x}) \in \mathbb{R}^{d}, p_{\delta}:(t, \boldsymbol{x}) \in(0, T) \times \mathcal{D} \mapsto$ $p_{\delta}(t, \boldsymbol{x}) \in \mathbb{R}$ and $\boldsymbol{\sigma}_{\delta}:(t, \boldsymbol{x}) \in[0, T) \times \mathcal{D} \mapsto \boldsymbol{\sigma}_{\delta}(t, \boldsymbol{x}) \in \mathbb{R}_{S}^{d \times d}$ such that

$$
\begin{gathered}
\operatorname{Re}\left(\frac{\partial \boldsymbol{u}_{\delta}}{\partial t}+\left(\boldsymbol{u}_{\delta} \cdot \boldsymbol{\nabla}\right) \boldsymbol{u}_{\delta}\right)=-\boldsymbol{\nabla} p_{\delta}+(1-\varepsilon) \Delta \boldsymbol{u}_{\delta}+\frac{\varepsilon}{\mathrm{Wi}} \operatorname{div} \beta_{\delta}\left(\boldsymbol{\sigma}_{\delta}\right)+\boldsymbol{f} \\
\text { on } \mathcal{D}_{T},
\end{gathered}
$$




$$
\begin{array}{rlrl}
\operatorname{div} \boldsymbol{u}_{\delta} & =0 & & \text { on } \mathcal{D}_{T}, \\
\frac{\partial \boldsymbol{\sigma}_{\delta}}{\partial t}+\left(\boldsymbol{u}_{\delta} \cdot \boldsymbol{\nabla}\right) \boldsymbol{\sigma}_{\delta} & =\left(\boldsymbol{\nabla} \boldsymbol{u}_{\delta}\right) \beta_{\delta}\left(\boldsymbol{\sigma}_{\delta}\right)+\beta_{\delta}\left(\boldsymbol{\sigma}_{\delta}\right)\left(\boldsymbol{\nabla} \boldsymbol{u}_{\delta}\right)^{T}-\frac{1}{\mathrm{Wi}}\left(\boldsymbol{\sigma}_{\delta}-\boldsymbol{I}\right) \\
\boldsymbol{u}_{\delta}(0, \boldsymbol{x}) & =\boldsymbol{u}^{0}(\boldsymbol{x}) & & \text { on } \mathcal{D}_{T} \\
\boldsymbol{\sigma}_{\delta}(0, \boldsymbol{x}) & =\boldsymbol{\sigma}^{0}(\boldsymbol{x}) & & \forall \boldsymbol{x} \in \mathcal{D} \\
\boldsymbol{u}_{\delta} & =\mathbf{0} & & \forall \boldsymbol{x} \in \mathcal{D}, \\
& \text { on }(0, T) \times \partial \mathcal{D} .
\end{array}
$$

\subsection{Formal energy estimates for $\left(\boldsymbol{P}_{\delta}\right)$}

In this section, we derive formal energy estimates, see e.g. (2.21) below, where we will assume that the triple $\left(\boldsymbol{u}_{\delta}, p_{\delta}, \boldsymbol{\sigma}_{\delta}\right)$, which is a solution to problem $\left(\mathrm{P}_{\delta}\right)$, has sufficient regularity for all the subsequent manipulations.

We will assume throughout that

$\boldsymbol{f} \in L^{2}\left(0, T ;\left[H^{-1}(\mathcal{D})\right]^{d}\right), \quad \boldsymbol{u}^{0} \in\left[L^{2}(\mathcal{D})\right]^{d}, \quad$ and $\quad \boldsymbol{\sigma}^{0} \in\left[L^{\infty}(\mathcal{D})\right]_{S P D}^{d \times d}$

with $\quad \sigma_{\min }^{0}\|\boldsymbol{\xi}\|^{2} \leq \boldsymbol{\xi}^{T} \boldsymbol{\sigma}^{0}(\boldsymbol{x}) \boldsymbol{\xi} \leq \sigma_{\max }^{0}\|\boldsymbol{\xi}\|^{2} \quad \forall \boldsymbol{\xi} \in \mathbb{R}^{d} \quad$ for a.e. $\boldsymbol{x}$ in $\mathcal{D}$;

where $\sigma_{\min }^{0}, \sigma_{\max }^{0} \in \mathbb{R}_{>0}$.

Let $F_{\delta}\left(\boldsymbol{u}_{\delta}, \boldsymbol{\sigma}_{\delta}\right)$ denote the free energy of the solution $\left(\boldsymbol{u}_{\delta}, p_{\delta}, \boldsymbol{\sigma}_{\delta}\right)$ to problem $\left(\mathrm{P}_{\delta}\right)$, where $F_{\delta}(\cdot, \cdot): \mathrm{W} \times \mathrm{S} \rightarrow \mathbb{R}$ is defined as

$$
F_{\delta}(\boldsymbol{v}, \phi):=\frac{\operatorname{Re}}{2} \int_{\mathcal{D}}\|\boldsymbol{v}\|^{2}+\frac{\varepsilon}{2 \mathrm{Wi}} \int_{\mathcal{D}} \operatorname{tr}\left(\boldsymbol{\phi}-G_{\delta}(\phi)-\boldsymbol{I}\right) .
$$

Here the first term $\frac{\operatorname{Re}}{2} \int_{\mathcal{D}}\|\boldsymbol{v}\|^{2}$ corresponds to the usual kinetic energy term, and the second term $\frac{\varepsilon}{2 \mathrm{Wi}} \int_{\mathcal{D}} \operatorname{tr}\left(\boldsymbol{\phi}-G_{\delta}(\boldsymbol{\phi})-\boldsymbol{I}\right)$ is a regularized version of the relative entropy term $\frac{\varepsilon}{2 \mathrm{Wi}} \int_{\mathcal{D}} \operatorname{tr}(\boldsymbol{\phi}-G(\boldsymbol{\phi})-\boldsymbol{I})$ introduced in Hu and Lelièvre, $\stackrel{\text { HL07 }}{\text { see also }}$ Jourdain et al. JLLO06.

Proposition 2.1. Let $\left(\boldsymbol{u}_{\delta}, p_{\delta}, \boldsymbol{\sigma}_{\delta}\right)$ be a sufficiently smooth solution to problem $\left(\mathrm{P}_{\delta}\right)$. Then the free energy $F_{\delta}\left(\boldsymbol{u}_{\delta}, \boldsymbol{\sigma}_{\delta}\right)$ satisfies for a.e. $t \in(0, T)$

$$
\begin{aligned}
\frac{d}{d t} F_{\delta}\left(\boldsymbol{u}_{\delta}, \boldsymbol{\sigma}_{\delta}\right)+(1-\varepsilon) \int_{\mathcal{D}}\left\|\boldsymbol{\nabla} \boldsymbol{u}_{\delta}\right\|^{2}+\frac{\varepsilon}{2 \mathrm{Wi}^{2}} \int_{\mathcal{D}} \operatorname{tr}\left(\beta_{\delta}\left(\boldsymbol{\sigma}_{\delta}\right)+\right. & {\left.\left[\beta_{\delta}\left(\boldsymbol{\sigma}_{\delta}\right)\right]^{-1}-2 \boldsymbol{I}\right) } \\
& \leq\left\langle\boldsymbol{f}, \boldsymbol{u}_{\delta}\right\rangle_{H_{0}^{1}(\mathcal{D})} .
\end{aligned}
$$

Proof. Multiplying the Navier-Stokes equation with $\boldsymbol{u}_{\delta}$ and the stress equation with $\frac{\varepsilon}{2 \mathrm{Wi}}\left(\boldsymbol{I}-G_{\delta}^{\prime}\left(\boldsymbol{\sigma}_{\delta}\right)\right)$, summing and integrating over $\mathcal{D}$ yields, after using integrations by parts and the incompressibility property in the standard way, that

$$
\begin{aligned}
\int_{\mathcal{D}} & {\left[\frac{\mathrm{Re}}{2} \frac{\partial}{\partial t}\left\|\boldsymbol{u}_{\delta}\right\|^{2}+(1-\varepsilon)\left\|\boldsymbol{\nabla} \boldsymbol{u}_{\delta}\right\|^{2}+\frac{\varepsilon}{\mathrm{Wi}} \beta_{\delta}\left(\boldsymbol{\sigma}_{\delta}\right): \boldsymbol{\nabla} \boldsymbol{u}_{\delta}\right] } \\
+ & \frac{\varepsilon}{2 \mathrm{Wi}} \int_{\mathcal{D}}\left[\left(\frac{\partial}{\partial t} \boldsymbol{\sigma}_{\delta}+\left(\boldsymbol{u}_{\delta} \cdot \boldsymbol{\nabla}\right) \boldsymbol{\sigma}_{\delta}\right)+\frac{1}{\mathrm{Wi}}\left(\boldsymbol{\sigma}_{\delta}-\boldsymbol{I}\right)\right]:\left(\boldsymbol{I}-G_{\delta}^{\prime}\left(\boldsymbol{\sigma}_{\delta}\right)\right)
\end{aligned}
$$




$$
-\frac{\varepsilon}{2 \mathrm{Wi}} \int_{\mathcal{D}}\left(\left(\boldsymbol{\nabla} \boldsymbol{u}_{\delta}\right) \beta_{\delta}\left(\boldsymbol{\sigma}_{\delta}\right)+\beta_{\delta}\left(\boldsymbol{\sigma}_{\delta}\right)\left(\boldsymbol{\nabla} \boldsymbol{u}_{\delta}\right)^{T}\right):\left(\boldsymbol{I}-G_{\delta}^{\prime}\left(\boldsymbol{\sigma}_{\delta}\right)\right)=\left\langle\boldsymbol{f}, \boldsymbol{u}_{\delta}\right\rangle_{H_{0}^{1}(\mathcal{D})}
$$

Using (2.16) and its spatial counterpart, we first note that

$$
\left(\frac{\partial}{\partial t} \boldsymbol{\sigma}_{\delta}+\left(\boldsymbol{u}_{\delta} \cdot \boldsymbol{\nabla}\right) \boldsymbol{\sigma}_{\delta}\right):\left(\boldsymbol{I}-G_{\delta}^{\prime}\left(\boldsymbol{\sigma}_{\delta}\right)\right)=\left(\frac{\partial}{\partial t}+\left(\boldsymbol{u}_{\delta} \cdot \boldsymbol{\nabla}\right)\right) \operatorname{tr}\left(\boldsymbol{\sigma}_{\delta}-G_{\delta}\left(\boldsymbol{\sigma}_{\delta}\right)\right) .
$$

On integrating over $\mathcal{D}$, the $\left(\boldsymbol{u}_{\delta} \cdot \boldsymbol{\nabla}\right)$ part of this term vanishes as $\boldsymbol{u}_{\delta}(t, \cdot) \in \mathrm{V}$. On using trace properties, 2.7a and the incompressibility property, we obtain that

$$
\begin{aligned}
\left(\left(\boldsymbol{\nabla} \boldsymbol{u}_{\delta}\right) \beta_{\delta}\left(\boldsymbol{\sigma}_{\delta}\right)\right):\left(\boldsymbol{I}-G_{\delta}^{\prime}\left(\boldsymbol{\sigma}_{\delta}\right)\right) & =\operatorname{tr}\left(\left(\boldsymbol{\nabla} \boldsymbol{u}_{\delta}\right) \beta_{\delta}\left(\boldsymbol{\sigma}_{\delta}\right)-\left(\boldsymbol{\nabla} \boldsymbol{u}_{\delta}\right) \beta_{\delta}\left(\boldsymbol{\sigma}_{\delta}\right) G_{\delta}^{\prime}\left(\boldsymbol{\sigma}_{\delta}\right)\right) \\
& =\operatorname{tr}\left(\left(\boldsymbol{\nabla} \boldsymbol{u}_{\delta}\right) \beta_{\delta}\left(\boldsymbol{\sigma}_{\delta}\right)-\boldsymbol{\nabla} \boldsymbol{u}_{\delta}\right) \\
& =\operatorname{tr}\left(\left(\boldsymbol{\nabla} \boldsymbol{u}_{\delta}\right) \beta_{\delta}\left(\boldsymbol{\sigma}_{\delta}\right)\right)-\operatorname{div} \boldsymbol{u}_{\delta} \\
& =\operatorname{tr}\left(\left(\boldsymbol{\nabla} \boldsymbol{u}_{\delta}\right) \beta_{\delta}\left(\boldsymbol{\sigma}_{\delta}\right)\right)
\end{aligned}
$$

On noting (1.4a), we have also that

$$
\left(\beta_{\delta}\left(\boldsymbol{\sigma}_{\delta}\right)\left(\boldsymbol{\nabla} \boldsymbol{u}_{\delta}\right)^{T}\right):\left(\boldsymbol{I}-G_{\delta}^{\prime}\left(\boldsymbol{\sigma}_{\delta}\right)\right)=\operatorname{tr}\left(\left(\boldsymbol{\nabla} \boldsymbol{u}_{\delta}\right) \beta_{\delta}\left(\boldsymbol{\sigma}_{\delta}\right)\right) .
$$

Therefore the terms involving the left-hand sides of (2.24) and (2.25) in (2.22) cancel with the term $\frac{\varepsilon}{\mathrm{Wi}} \beta_{\delta}\left(\boldsymbol{\sigma}_{\delta}\right): \boldsymbol{\nabla} \boldsymbol{u}_{\delta}$ in (2.22) arising from the Navier-Stokes equation. Finally, for the remaining term we have on noting (1.2b) and (2.7ad) that

$$
\begin{aligned}
\left(\boldsymbol{\sigma}_{\delta}-\boldsymbol{I}\right):\left(\boldsymbol{I}-G_{\delta}^{\prime}\left(\boldsymbol{\sigma}_{\delta}\right)\right) & =\left[\left(\boldsymbol{\sigma}_{\delta}-\beta_{\delta}\left(\boldsymbol{\sigma}_{\delta}\right)\right)+\left(\beta_{\delta}\left(\boldsymbol{\sigma}_{\delta}\right)-\boldsymbol{I}\right)\right]:\left(\boldsymbol{I}-G_{\delta}^{\prime}\left(\boldsymbol{\sigma}_{\delta}\right)\right) \\
& \geq\left(\beta_{\delta}\left(\boldsymbol{\sigma}_{\delta}\right)-\boldsymbol{I}\right):\left(\boldsymbol{I}-G_{\delta}^{\prime}\left(\boldsymbol{\sigma}_{\delta}\right)\right) \\
& =\operatorname{tr}\left(\beta_{\delta}\left(\boldsymbol{\sigma}_{\delta}\right)+\left[\beta_{\delta}\left(\boldsymbol{\sigma}_{\delta}\right)\right]^{-1}-2 \boldsymbol{I}\right) .
\end{aligned}
$$

Hence we obtain the desired free energy inequality (2.21).

Corollary 2.1. Let $\left(\boldsymbol{u}_{\delta}, p_{\delta}, \boldsymbol{\sigma}_{\delta}\right)$ be a sufficiently smooth solution to problem $\left(\mathrm{P}_{\delta}\right)$. Then it follows that

$$
\begin{aligned}
& \sup _{t \in(0, T)} F_{\delta}\left(\boldsymbol{u}_{\delta}(t, \cdot), \boldsymbol{\sigma}_{\delta}(t, \cdot)\right) \\
&+\int_{\mathcal{D}_{T}}\left[\frac{1}{2}(1-\varepsilon)\left\|\boldsymbol{\nabla} \boldsymbol{u}_{\delta}\right\|^{2}+\frac{\varepsilon}{2 \mathrm{Wi}^{2}} \operatorname{tr}\left(\beta_{\delta}\left(\boldsymbol{\sigma}_{\delta}\right)+\left[\beta_{\delta}\left(\boldsymbol{\sigma}_{\delta}\right)\right]^{-1}-2 \boldsymbol{I}\right)\right] \\
& \leq 2\left(F_{\delta}\left(\boldsymbol{u}^{0}, \boldsymbol{\sigma}^{0}\right)+\frac{1+C_{P}}{2(1-\varepsilon)}\|\boldsymbol{f}\|_{L^{2}\left(0, T ; H^{-1}(\mathcal{D})\right)}^{2}\right) .
\end{aligned}
$$

Proof. Smooth solutions $\left(\boldsymbol{u}_{\delta}, p_{\delta}, \boldsymbol{\sigma}_{\delta}\right)$ of $\left(\mathrm{P}_{\delta}\right)$ satisfy the free energy estimate (2.20). One can bound the term $\left\langle\boldsymbol{f}, \boldsymbol{u}_{\delta}\right\rangle_{H_{0}^{1}(\mathcal{D})}$ there, using the Cauchy-Schwarz and Young inequalities for $\nu \in \mathbb{R}_{>0}$, and the Poincaré inequality (1.8), by

$$
\left\langle\boldsymbol{f}, \boldsymbol{u}_{\delta}\right\rangle_{H_{0}^{1}(\mathcal{D})} \leq\|\boldsymbol{f}\|_{H^{-1}(\mathcal{D})}\left\|\boldsymbol{u}_{\delta}\right\|_{H^{1}(\mathcal{D})} \leq \frac{1}{2 \nu^{2}}\|\boldsymbol{f}\|_{H^{-1}(\mathcal{D})}^{2}+\frac{\nu^{2}}{2}\left\|\boldsymbol{u}_{\delta}\right\|_{H^{1}(\mathcal{D})}^{2}
$$




$$
\leq \frac{1}{2 \nu^{2}}\|\boldsymbol{f}\|_{H^{-1}(\mathcal{D})}^{2}+\frac{\nu^{2}}{2}\left(1+C_{P}\right)\left\|\boldsymbol{\nabla} \boldsymbol{u}_{\delta}\right\|_{L^{2}(\mathcal{D})}^{2} .
$$

Combining (2.28) and (2.20) with $\nu^{2}=(1-\varepsilon) /\left(1+C_{P}\right)$, and integrating in time yields the desired result (2.27).

We note that the right-hand side of (2.27) is independent of the regularization parameter $\delta$ if $\boldsymbol{\sigma}^{0}$ is positive definite.

\section{Finite element approximation of $\left(\mathrm{P}_{\delta}\right)$ and $(\mathrm{P})$}

\subsection{Finite element discretization}

We now introduce a finite element discretization of the problem $\left(\mathrm{P}_{\delta}\right)$, which satisfies a discrete analogue of (2.21).

The time interval $[0, T)$ is split into intervals $\left[t^{n-1}, t^{n}\right)$ with $\Delta t_{n}=t^{n}-t^{n-1}$, $n=1, \ldots, N_{T}$. We set $\Delta t:=\max _{n=1, \ldots, N_{T}} \Delta t_{n}$. We will assume throughout that the domain $\mathcal{D}$ is a polytope. We define a regular family of meshes $\left\{\mathcal{T}_{h}\right\}_{h>0}$ with discretization parameter $h>0$, which is built from partitionings of the domain $\mathcal{D}$ into regular open simplices so that

$$
\overline{\mathcal{D}}=\mathcal{T}_{h}:=\bigcup_{k=1}^{N_{K}} \overline{K_{k}} \quad \text { with } \quad \max _{k=1, \ldots, N_{K}} \frac{h_{k}}{\rho_{k}} \leq C .
$$

Here $\rho_{k}$ is the diameter of the largest inscribed ball contained in the simplex $K_{k}$ and $h_{k}$ is the diameter of $K_{k}$, so that $h=\max _{k=1, \ldots, N_{K}} h_{k}$. For each element $K_{k}, k=1, \ldots, N_{K}$, of the mesh $\mathcal{T}_{h}$ let $\left\{P_{i}^{k}\right\}_{i=0}^{d}$ denotes its vertices, and $\left\{\boldsymbol{n}_{i}^{k}\right\}_{i=0}^{d}$ the outward unit normals of the edges $(d=2)$ or faces $(d=3)$ with $\boldsymbol{n}_{i}^{k}$ being that of the edge/face opposite vertex $P_{i}^{k}, i=0, \ldots, d$. In addition, let $\left\{\eta_{i}^{k}(\boldsymbol{x})\right\}_{i=0}^{d}$ denote the barycentric coordinates of $\boldsymbol{x} \in K_{k}$ with respect to the vertices $\left\{P_{i}^{k}\right\}_{i=0}^{d}$; that is, $\eta_{i}^{k} \in \mathbb{P}_{1}$ and $\eta_{i}^{k}\left(P_{j}^{k}\right)=\delta_{i j}, i, j=0, \ldots, d$. Here $\mathbb{P}_{m}$ denote polynomials of maximal degree $m$ in $\boldsymbol{x}$, and $\delta_{i j}$ the Kronecker delta notation. Finally, we introduce $\partial \mathcal{T}_{h}:=\left\{E_{j}\right\}_{j=1}^{N_{E}}$ as the set of internal edges $E_{j}$ of triangles in the mesh $\mathcal{T}_{h}$ when $d=2$, or the set of internal faces $E_{j}$ of tetrahedra when $d=3$.

We approximate the problem $\left(\mathrm{P}_{\delta}\right)$ by the problem $\left(\mathrm{P}_{\delta, h}^{\Delta t}\right)$ based on the finite element spaces $\mathrm{W}_{h}^{0} \times \mathrm{Q}_{h}^{0} \times \mathrm{S}_{h}^{0}$. As is standard, we require the discrete velocitypressure spaces $\mathrm{W}_{h}^{0} \times \mathrm{Q}_{h}^{0} \subset \mathrm{W} \times \mathrm{Q}$ satisfy the discrete Ladyshenskaya-BabuškaBrezzi (LBB) inf-sup condition

$$
\inf _{q \in \mathrm{Q}_{h}^{0}} \sup _{\boldsymbol{v} \in \mathrm{W}_{h}^{0}} \frac{\int_{\mathcal{D}} q \operatorname{div} \boldsymbol{v}}{\|q\|_{L^{2}(\mathcal{D})}\|\boldsymbol{v}\|_{H^{1}(\mathcal{D})}} \geq \mu_{\star}>0,
$$

see e.g. p114 in Girault and Raviart GR86 In the following, we set

$$
\begin{aligned}
\mathrm{W}_{h}^{0} & :=\mathrm{W}_{h}^{2} \subset \mathrm{W} \quad \text { or } \quad \mathrm{W}_{h}^{2,-} \subset \mathrm{W}, \\
\mathrm{Q}_{h}^{0} & :=\left\{q \in \mathrm{Q}:\left.q\right|_{K_{k}} \in \mathbb{P}_{0} \quad k=1, \ldots, N_{K}\right\} \subset \mathrm{Q},
\end{aligned}
$$


and $\quad \mathrm{S}_{h}^{0}:=\left\{\boldsymbol{\phi} \in \mathrm{S}:\left.\boldsymbol{\phi}\right|_{K_{k}} \in\left[\mathbb{P}_{0}\right]_{S}^{d \times d} \quad k=1, \ldots, N_{K}\right\} \subset \mathrm{S} ;$

where

$$
\begin{aligned}
\mathrm{W}_{h}^{2} & :=\left\{\boldsymbol{v} \in[C(\overline{\mathcal{D}})]^{d} \cap \mathrm{W}:\left.\boldsymbol{v}\right|_{K_{k}} \in\left[\mathbb{P}_{2}\right]^{d} \quad k=1, \ldots, N_{K}\right\} \\
\mathrm{W}_{h}^{2,-} & :=\left\{\boldsymbol{v} \in[C(\overline{\mathcal{D}})]^{d} \cap \mathrm{W}:\left.\boldsymbol{v}\right|_{K_{k}} \in\left[\mathbb{P}_{1}\right]^{d} \oplus \operatorname{span}\left\{\boldsymbol{\varsigma}_{i}^{k}\right\}_{i=0}^{d} \quad k=1, \ldots, N_{K}\right\} .
\end{aligned}
$$

Here, for $k=1, \ldots, N_{K}$ and $i=0, \ldots, d$

$$
\boldsymbol{\varsigma}_{i}^{k}(\boldsymbol{x})=\boldsymbol{n}_{i}^{k} \prod_{j=0, j \neq i}^{d} \eta_{j}^{k}(\boldsymbol{x}) \quad \text { for } \boldsymbol{x} \in K_{k} .
$$

We introduce also

$$
\mathrm{V}_{h}^{0}:=\left\{\boldsymbol{v} \in \mathrm{W}_{h}^{0}: \int_{\mathcal{D}} q \operatorname{div} \boldsymbol{v}=0 \quad \forall q \in \mathrm{Q}_{h}^{0}\right\},
$$

which approximates V. It is well-known the choices (3.2a,b) satisfies (3.1), see e.g. p221 in Brezzi and Fortin $\frac{B F 92}{\text { for }} \mathrm{W}_{h}^{0}=\mathrm{W}_{h}^{2}$, and Chapter II, Sections $2.1(d=2)$ and $2.3(d=3)$ in Girault and Raviart ${ }^{\mathrm{GR} 86}$ for $\mathrm{W}_{h}^{0}=\mathrm{W}_{h}^{2,-}$. Moreover, these particular choices of $\mathrm{S}_{h}^{0}$ and $\mathrm{Q}_{h}^{0}$ have the desirable property that

$$
\phi \in \mathrm{S}_{h}^{0} \quad \Rightarrow \quad \boldsymbol{I}-G_{\delta}^{\prime}(\phi) \in \mathrm{S}_{h}^{0} \quad \text { and } \quad \operatorname{tr}\left(\boldsymbol{\phi}-G_{\delta}(\phi)-\boldsymbol{I}\right) \in \mathrm{Q}_{h}^{0} \text {, }
$$

which makes it a straightforward matter to mimic the free energy inequality (2.21) at a discrete level. Since $S_{h}^{0}$ is discontinuous, we will use the discontinuous Galerkin method to approximate the advection term $\left(\boldsymbol{u}_{\delta} \cdot \nabla\right) \boldsymbol{\sigma}_{\delta}$ in the following. Then, for the boundary integrals, we will make use of the following definitions (see e.g. p267 in Ern and Guermond ${ }^{E G 04}$ ). Given $\boldsymbol{v} \in \mathrm{W}_{h}^{0}$, then for any $\phi \in \mathrm{S}_{h}^{0}$ (or $\mathrm{Q}_{h}^{0}$ ) and for any point $\boldsymbol{x}$ that is in the interior of some $E_{j} \in \partial \mathcal{T}_{h}$, we define the downstream and upstream values of $\phi$ at $\boldsymbol{x}$ by

$$
\phi^{+\boldsymbol{v}}(\boldsymbol{x})=\lim _{\rho \rightarrow 0^{+}} \phi(\boldsymbol{x}+\rho \boldsymbol{v}(\boldsymbol{x})) \quad \text { and } \quad \phi^{-\boldsymbol{v}}(\boldsymbol{x})=\lim _{\rho \rightarrow 0^{-}} \phi(\boldsymbol{x}+\rho \boldsymbol{v}(\boldsymbol{x})) ;
$$

respectively. In addition, we denote by

$$
\llbracket \phi \rrbracket_{\rightarrow \boldsymbol{v}}(\boldsymbol{x})=\boldsymbol{\phi}^{+\boldsymbol{v}}(\boldsymbol{x})-\boldsymbol{\phi}^{-\boldsymbol{v}}(\boldsymbol{x}) \quad \text { and } \quad\{\phi\}^{\boldsymbol{v}}(\boldsymbol{x})=\frac{\phi^{+\boldsymbol{v}}(\boldsymbol{x})+\phi^{-\boldsymbol{v}}(\boldsymbol{x})}{2},
$$

the jump and mean value, respectively, of $\boldsymbol{\phi}$ at the point $\boldsymbol{x}$ of boundary $E_{j}$. From (3.6), it is clear that the values of $\left.\boldsymbol{\phi}^{+\boldsymbol{v}}\right|_{E_{j}}$ and $\left.\boldsymbol{\phi}^{-\boldsymbol{v}}\right|_{E_{j}}$ can change along $E_{j} \in \partial \mathcal{T}_{h}$. Finally, it is easily deduced that

$$
\begin{aligned}
\sum_{j=1}^{N_{E}} \int_{E_{j}}|\boldsymbol{v} \cdot \boldsymbol{n}| \llbracket q_{1} \rrbracket \rightarrow \boldsymbol{v} q_{2}^{+\boldsymbol{v}}=-\sum_{k=1}^{N_{K}} \int_{\partial K_{k}}\left(\boldsymbol{v} \cdot \boldsymbol{n}_{K_{k}}\right) q_{1} q_{2}^{+\boldsymbol{v}} \\
\forall \boldsymbol{v} \in \mathrm{W}_{h}^{0}, q_{1}, q_{2} \in \mathrm{Q}_{h}^{0} ;
\end{aligned}
$$

where $\boldsymbol{n} \equiv \boldsymbol{n}\left(E_{j}\right)$ is a unit normal to $E_{j}$, whose sign is of no importance, and $\boldsymbol{n}_{K_{k}}$ is the outward unit normal vector of boundary $\partial K_{k}$ of $K_{k}$. We note that similar ideas appear in upwind schemes; e.g. see Chapter IV, Section 5 in Girault and Raviart GR86 for the Navier-Stokes equations. 


\subsection{A free energy preserving approximation, $\left(\boldsymbol{P}_{\delta, h}^{\Delta t}\right)$, of $\left(\boldsymbol{P}_{\delta}\right)$}

For any source term $\boldsymbol{f} \in L^{2}\left(0, T ;\left[H^{-1}(\mathcal{D})\right]^{d}\right)$, we define the following piecewise constant function with respect to the time variable

$$
\boldsymbol{f}^{\Delta t,+}(t, \cdot)=\boldsymbol{f}^{n}(\cdot):=\frac{1}{\Delta t_{n}} \int_{t^{n-1}}^{t^{n}} \boldsymbol{f}(t, \cdot) d t, \quad t \in\left[t^{n-1}, t^{n}\right), \quad n=1, \ldots, N_{T} .
$$

It is easily deduced that

$$
\sum_{n=1}^{N_{T}} \Delta t_{n}\left\|\boldsymbol{f}^{n}\right\|_{H^{-1}(\mathcal{D})}^{r} \leq \int_{0}^{T}\|f(t, \cdot)\|_{H^{-1}(\mathcal{D})}^{r} d t \quad \text { for any } r \in[1,2],
$$

and $\quad \boldsymbol{f}^{\Delta t,+} \rightarrow \boldsymbol{f} \quad$ strongly in $L^{2}\left(0, T ;\left[H^{-1}(\mathcal{D})\right]^{d}\right)$ as $\Delta t \rightarrow 0_{+}$.

Throughout this section we choose $\boldsymbol{u}_{h}^{0} \in \mathrm{V}_{h}^{0}$ to be a suitable approximation of $\boldsymbol{u}^{0}$ such as the $L^{2}$ projection of $\boldsymbol{u}^{0}$ onto $\mathrm{V}_{h}^{0}$. We will also choose $\boldsymbol{\sigma}_{h}^{0} \in \mathrm{S}_{h}^{0}$ to be the $L^{2}$ projection of $\boldsymbol{\sigma}^{0}$ onto $\mathrm{S}_{h}^{0}$. Hence for $k=1, \ldots, N_{K}$

$$
\left.\boldsymbol{\sigma}_{h}^{0}\right|_{K_{k}}=\frac{1}{\left|K_{k}\right|} \int_{K_{k}} \boldsymbol{\sigma}^{0},
$$

where $\left|K_{k}\right|$ is the measure of $K_{k}$; and it immediately follows from (2.19) that

$$
\sigma_{\min }^{0}\|\boldsymbol{\xi}\|^{2} \leq\left.\boldsymbol{\xi}^{T} \boldsymbol{\sigma}_{h}^{0}\right|_{K_{k}} \boldsymbol{\xi} \leq \sigma_{\max }^{0}\|\boldsymbol{\xi}\|^{2} \quad \forall \boldsymbol{\xi} \in \mathbb{R}^{d} .
$$

Our approximation $\left(\mathrm{P}_{\delta, h}^{\Delta t}\right)$ of $\left(\mathrm{P}_{\delta}\right)$ is then:

$\left(\mathbf{P}_{\delta, h}^{\Delta t}\right)$ Setting $\left(\boldsymbol{u}_{\delta, h}^{0}, \boldsymbol{\sigma}_{\delta, h}^{0}\right)=\left(\boldsymbol{u}_{h}^{0}, \boldsymbol{\sigma}_{h}^{0}\right) \in \mathrm{V}_{h}^{0} \times \mathrm{S}_{h}^{0}$, then for $n=1, \ldots, N_{T}$ find $\left(\boldsymbol{u}_{\delta, h}^{n}, \boldsymbol{\sigma}_{\delta, h}^{n}\right) \in \mathrm{V}_{h}^{0} \times \mathrm{S}_{h}^{0}$ such that for any test functions $(\boldsymbol{v}, \boldsymbol{\phi}) \in \mathrm{V}_{h}^{0} \times \mathrm{S}_{h}^{0}$

$$
\begin{gathered}
\int_{\mathcal{D}}\left[\operatorname{Re}\left(\frac{\boldsymbol{u}_{\delta, h}^{n}-\boldsymbol{u}_{\delta, h}^{n-1}}{\Delta t_{n}}\right) \cdot \boldsymbol{v}+\frac{\operatorname{Re}}{2}\left[\left(\left(\boldsymbol{u}_{\delta, h}^{n-1} \cdot \nabla\right) \boldsymbol{u}_{\delta, h}^{n}\right) \cdot \boldsymbol{v}-\boldsymbol{u}_{\delta, h}^{n} \cdot\left(\left(\boldsymbol{u}_{\delta, h}^{n-1} \cdot \nabla\right) \boldsymbol{v}\right)\right]\right. \\
\left.+(1-\varepsilon) \boldsymbol{\nabla} \boldsymbol{u}_{\delta, h}^{n}: \boldsymbol{\nabla} \boldsymbol{v}+\frac{\varepsilon}{\mathrm{Wi}} \beta_{\delta}\left(\boldsymbol{\sigma}_{\delta, h}^{n}\right): \boldsymbol{\nabla} \boldsymbol{v}\right]=\left\langle\boldsymbol{f}^{n}, \boldsymbol{v}\right\rangle_{H_{0}^{1}(\mathcal{D})},(3.12 \\
\int_{\mathcal{D}}\left[\left(\frac{\boldsymbol{\sigma}_{\delta, h}^{n}-\boldsymbol{\sigma}_{\delta, h}^{n-1}}{\Delta t_{n}}\right): \boldsymbol{\phi}-2\left(\left(\boldsymbol{\nabla} \boldsymbol{u}_{\delta, h}^{n}\right) \beta_{\delta}\left(\boldsymbol{\sigma}_{\delta, h}^{n}\right)\right): \boldsymbol{\phi}+\frac{1}{\mathrm{Wi}}\left(\boldsymbol{\sigma}_{\delta, h}^{n}-\boldsymbol{I}\right): \boldsymbol{\phi}\right] \\
+\sum_{j=1}^{N_{E}} \int_{E_{j}}\left|\boldsymbol{u}_{\delta, h}^{n-1} \cdot \boldsymbol{n}\right| \llbracket \boldsymbol{\sigma}_{\delta, h}^{n} \rrbracket_{\rightarrow \boldsymbol{u}_{\delta, h}^{n-1}}: \boldsymbol{\phi}^{+\boldsymbol{u}_{\delta, h}^{n-1}}=0
\end{gathered}
$$

In deriving $\left(\mathrm{P}_{\delta, h}^{\Delta t}\right)$, we have noted (1.4a) and that

$$
\int_{\mathcal{D}} \boldsymbol{v} \cdot[(\boldsymbol{z} \cdot \nabla) \boldsymbol{w}]=-\int_{\mathcal{D}} \boldsymbol{w} \cdot[(\boldsymbol{z} \cdot \nabla) \boldsymbol{v}] \quad \forall \boldsymbol{z} \in \mathrm{V}, \quad \forall \boldsymbol{v}, \boldsymbol{w} \in\left[H^{1}(\mathcal{D})\right]^{d} .
$$

Once again we refer to p267 in Ern and Guermond $\underline{\text { EG04 }}$ for the consistency of our stated approximation of the stress convection term, see also Boyaval et al. BLM09. 
Before proving existence of a solution to $\left(\mathrm{P}_{\delta, h}^{\Delta t}\right)$, we first derive a discrete analogue of the energy estimate (2.21) for $\left(\mathrm{P}_{\delta, h}^{\Delta t}\right)$; which uses the elementary equality

$$
2 s_{1}\left(s_{1}-s_{2}\right)=s_{1}^{2}-s_{2}^{2}+\left(s_{1}-s_{2}\right)^{2} \quad \forall s_{1}, s_{2} \in \mathbb{R} .
$$

\subsection{Energy bound for $\left(P_{\delta, h}^{\Delta t}\right)$}

Proposition 3.1. For $n=1, \ldots, N_{T}$, a solution $\left(\boldsymbol{u}_{\delta, h}^{n}, \boldsymbol{\sigma}_{\delta, h}^{n}\right) \in \mathrm{V}_{h}^{0} \times \mathrm{S}_{h}^{0}$ to (3.12a, b), if it exists, satisfies

$$
\begin{gathered}
\frac{F_{\delta}\left(\boldsymbol{u}_{\delta, h}^{n}, \boldsymbol{\sigma}_{\delta, h}^{n}\right)-F_{\delta}\left(\boldsymbol{u}_{\delta, h}^{n-1}, \boldsymbol{\sigma}_{\delta, h}^{n-1}\right)}{\Delta t_{n}}+\frac{\operatorname{Re}}{2 \Delta t_{n}} \int_{\mathcal{D}}\left\|\boldsymbol{u}_{\delta, h}^{n}-\boldsymbol{u}_{\delta, h}^{n-1}\right\|^{2}+(1-\varepsilon) \int_{\mathcal{D}}\left\|\boldsymbol{\nabla} \boldsymbol{u}_{\delta, h}^{n}\right\|^{2} \\
\quad+\frac{\varepsilon}{2 \mathrm{Wi}^{2}} \int_{\mathcal{D}} \operatorname{tr}\left(\beta_{\delta}\left(\boldsymbol{\sigma}_{\delta, h}^{n}\right)+\left[\beta_{\delta}\left(\boldsymbol{\sigma}_{\delta, h}^{n}\right)\right]^{-1}-2 \boldsymbol{I}\right) \\
\leq\left\langle\boldsymbol{f}^{n}, \boldsymbol{u}_{\delta, h}^{n}\right\rangle_{H_{0}^{1}(\mathcal{D})} \leq \frac{1}{2}(1-\varepsilon) \int_{\mathcal{D}}\left\|\boldsymbol{\nabla} \boldsymbol{u}_{\delta, h}^{n}\right\|^{2}+\frac{1+C_{P}}{2(1-\varepsilon)}\left\|\boldsymbol{f}^{n}\right\|_{H^{-1}(\mathcal{D})}^{2}
\end{gathered}
$$

Proof. Similarly to the proof of Proposition 2.1, we choose as test functions $\boldsymbol{v}=$ $\boldsymbol{u}_{\delta, h}^{n} \in \mathrm{V}_{h}^{0}$ and $\boldsymbol{\phi}=\frac{\varepsilon}{2 \mathrm{Wi}}\left(\boldsymbol{I}-G_{\delta}^{\prime}\left(\boldsymbol{\sigma}_{\delta, h}^{n}\right)\right) \in \mathrm{S}_{h}^{0}$ in (3.12a, b), and obtain, on noting (3.14) and (2.7a d), that

$$
\begin{aligned}
\left\langle\boldsymbol{f}^{n}, \boldsymbol{u}_{\delta, h}^{n}\right\rangle_{H_{0}^{1}(\mathcal{D})} & \int_{\mathcal{D}}\left[\frac{\operatorname{Re}}{2}\left(\frac{\left\|\boldsymbol{u}_{\delta, h}^{n}\right\|^{2}-\left\|\boldsymbol{u}_{\delta, h}^{n-1}\right\|^{2}}{\Delta t_{n}}+\frac{\left\|\boldsymbol{u}_{\delta, h}^{n}-\boldsymbol{u}_{\delta, h}^{n-1}\right\|^{2}}{\Delta t_{n}}\right)+(1-\varepsilon)\left\|\boldsymbol{\nabla} \boldsymbol{u}_{\delta, h}^{n}\right\|^{2}\right] \\
& +\frac{\varepsilon}{2 \mathrm{Wi}} \int_{\mathcal{D}}\left(\frac{\boldsymbol{\sigma}_{\delta, h}^{n}-\boldsymbol{\sigma}_{\delta, h}^{n-1}}{\Delta t_{n}}\right):\left(\boldsymbol{I}-G_{\delta}^{\prime}\left(\boldsymbol{\sigma}_{\delta, h}^{n}\right)\right) \\
& +\frac{\varepsilon}{2 \mathrm{Wi}^{2}} \int_{\mathcal{D}} \operatorname{tr}\left(\beta_{\delta}\left(\boldsymbol{\sigma}_{\delta, h}^{n}\right)+\left[\beta_{\delta}\left(\boldsymbol{\sigma}_{\delta, h}^{n}\right)\right]^{-1}-2 \boldsymbol{I}\right) \\
& +\frac{\varepsilon}{2 \mathrm{Wi}} \sum_{j=1}^{N_{E}} \int_{E_{j}}\left[\left|\boldsymbol{u}_{\delta, h}^{n-1} \cdot \boldsymbol{n}\right| \llbracket \boldsymbol{\sigma}_{\delta, h}^{n} \rrbracket_{\rightarrow \boldsymbol{u}_{\delta, h}^{n-1}}:\left(\boldsymbol{I}-G_{\delta}^{\prime}\left(\boldsymbol{\sigma}_{\delta, h}^{n}\right)\right)^{+\boldsymbol{u}_{\delta, h}^{n-1}}\right] .
\end{aligned}
$$

We consequently obtain from (3.16), on noting (1.2b) and (2.7e) applied to the edge terms as well as the discrete time derivative term for the stress variable, that

$$
\begin{aligned}
\left\langle\boldsymbol{f}^{n}, \boldsymbol{u}_{\delta, h}^{n}\right\rangle_{H_{0}^{1}(\mathcal{D})} & \geq \int_{\mathcal{D}}\left[\frac{\operatorname{Re}}{2}\left(\frac{\left\|\boldsymbol{u}_{\delta, h}^{n}\right\|^{2}-\left\|\boldsymbol{u}_{\delta, h}^{n-1}\right\|^{2}}{\Delta t_{n}}+\frac{\left\|\boldsymbol{u}_{\delta, h}^{n}-\boldsymbol{u}_{\delta, h}^{n-1}\right\|^{2}}{\Delta t_{n}}\right)+(1-\varepsilon)\left\|\boldsymbol{\nabla} \boldsymbol{u}_{\delta, h}^{n}\right\|^{2}\right] \\
& +\frac{\varepsilon}{2 \mathrm{Wi}} \int_{\mathcal{D}}\left(\frac{\operatorname{tr}\left(\boldsymbol{\sigma}_{\delta, h}^{n}-G_{\delta}\left(\boldsymbol{\sigma}_{\delta, h}^{n}\right)\right)-\operatorname{tr}\left(\boldsymbol{\sigma}_{\delta, h}^{n-1}-G_{\delta}\left(\boldsymbol{\sigma}_{\delta, h}^{n-1}\right)\right)}{\Delta t_{n}}\right) \\
& +\frac{\varepsilon}{2 \mathrm{Wi}^{2}} \int_{\mathcal{D}} \operatorname{tr}\left(\beta_{\delta}\left(\boldsymbol{\sigma}_{\delta, h}^{n}\right)+\left[\beta_{\delta}\left(\boldsymbol{\sigma}_{\delta, h}^{n}\right)\right]^{-1}-2 \boldsymbol{I}\right)
\end{aligned}
$$




$$
+\frac{\varepsilon}{2 \mathrm{Wi}} \sum_{j=1}^{N_{E}} \int_{E_{j}}\left|\boldsymbol{u}_{\delta, h}^{n-1} \cdot \boldsymbol{n}\right| \llbracket \operatorname{tr}\left(\boldsymbol{\sigma}_{\delta, h}^{n}-G_{\delta}\left(\boldsymbol{\sigma}_{\delta, h}^{n}\right)\right) \rrbracket_{\rightarrow \boldsymbol{u}_{\delta, h}^{n-1}} .
$$

Finally, we note from (3.8), (3.5) and as $\boldsymbol{u}_{\delta, h}^{n-1} \in \mathrm{V}_{h}^{0}$ that

$$
\begin{aligned}
\sum_{j=1}^{N_{E}} \int_{E_{j}}\left|\boldsymbol{u}_{\delta, h}^{n-1} \cdot \boldsymbol{n}\right| \llbracket \operatorname{tr}\left(\boldsymbol{\sigma}_{\delta, h}^{n}-G_{\delta}\left(\boldsymbol{\sigma}_{\delta, h}^{n}\right)\right) \rrbracket_{\rightarrow \boldsymbol{u}_{\delta, h}^{n-1}} \\
=-\sum_{k=1}^{N_{K}} \int_{\partial K_{k}}\left(\boldsymbol{u}_{\delta, h}^{n-1} \cdot \boldsymbol{n}_{K_{k}}\right) \operatorname{tr}\left(\boldsymbol{\sigma}_{\delta, h}^{n}-G_{\delta}\left(\boldsymbol{\sigma}_{\delta, h}^{n}\right)\right) \\
=-\sum_{k=1}^{N_{K}} \int_{K_{k}} \operatorname{div}\left(\boldsymbol{u}_{\delta, h}^{n-1} \operatorname{tr}\left(\boldsymbol{\sigma}_{\delta, h}^{n}-G_{\delta}\left(\boldsymbol{\sigma}_{\delta, h}^{n}\right)\right)\right) \\
=-\int_{\mathcal{D}} \operatorname{tr}\left(\boldsymbol{\sigma}_{\delta, h}^{n}-G_{\delta}\left(\boldsymbol{\sigma}_{\delta, h}^{n}\right)\right) \operatorname{div} \boldsymbol{u}_{\delta, h}^{n-1}=0 .
\end{aligned}
$$

Combining (3.17) and (3.18) yields the first desired inequality in (3.15). The second inequality in (3.15) follows immediately from (2.28) with $\nu^{2}=(1-\varepsilon) /\left(1+C_{P}\right)$.

\subsection{Existence of a solution to $\left(P_{\delta, h}^{\Delta t}\right)$}

Proposition 3.2. Given $\left(\boldsymbol{u}_{\delta, h}^{n-1}, \boldsymbol{\sigma}_{\delta, h}^{n-1}\right) \in \mathrm{V}_{h}^{0} \times \mathrm{S}_{h}^{0}$ and for any time step $\Delta t_{n}>0$, then there exists at least one solution $\left(\boldsymbol{u}_{\delta, h}^{n}, \boldsymbol{\sigma}_{\delta, h}^{n}\right) \in \mathrm{V}_{h}^{0} \times \mathrm{S}_{h}^{0}$ to (3.12a,,$\left.b\right)$.

Proof. We introduce the following inner product on the Hilbert space $\mathrm{V}_{h}^{0} \times \mathrm{S}_{h}^{0}$

$$
((\boldsymbol{w}, \boldsymbol{\psi}),(\boldsymbol{v}, \phi))_{\mathcal{D}}=\int_{\mathcal{D}}[\boldsymbol{w} \cdot \boldsymbol{v}+\boldsymbol{\psi}: \boldsymbol{\phi}] \quad \forall(\boldsymbol{w}, \boldsymbol{\psi}),(\boldsymbol{v}, \boldsymbol{\phi}) \in \mathrm{V}_{h}^{0} \times \mathrm{S}_{h}^{0} .
$$

Given $\left(\boldsymbol{u}_{\delta, h}^{n-1}, \boldsymbol{\sigma}_{\delta, h}^{n-1}\right) \in \mathrm{V}_{h}^{0} \times \mathrm{S}_{h}^{0}$, let $\mathcal{F}: \mathrm{V}_{h}^{0} \times \mathrm{S}_{h}^{0} \rightarrow \mathrm{V}_{h}^{0} \times \mathrm{S}_{h}^{0}$ be such that for any $(\boldsymbol{w}, \boldsymbol{\psi}) \in \mathrm{V}_{h}^{0} \times \mathrm{S}_{h}^{0}$

$$
\begin{aligned}
(\mathcal{F}(\boldsymbol{w}, \boldsymbol{\psi}),(\boldsymbol{v}, \boldsymbol{\phi}))_{\mathcal{D}} & \\
:=\int_{\mathcal{D}} & {\left[\operatorname{Re}\left(\frac{\boldsymbol{w}-\boldsymbol{u}_{\delta, h}^{n-1}}{\Delta t_{n}}\right) \cdot \boldsymbol{v}+\frac{\operatorname{Re}}{2}\left[\left(\left(\boldsymbol{u}_{\delta, h}^{n-1} \cdot \nabla\right) \boldsymbol{w}\right) \cdot \boldsymbol{v}-\boldsymbol{w} \cdot\left(\left(\boldsymbol{u}_{\delta, h}^{n-1} \cdot \nabla\right) \boldsymbol{v}\right)\right]\right.} \\
& +(1-\varepsilon) \boldsymbol{\nabla} \boldsymbol{w}: \boldsymbol{\nabla} \boldsymbol{v}+\frac{\varepsilon}{\mathrm{Wi}} \beta_{\delta}(\boldsymbol{\psi}): \boldsymbol{\nabla} \boldsymbol{v}+\left(\frac{\boldsymbol{\psi}-\boldsymbol{\sigma}_{\delta, h}^{n-1}}{\Delta t_{n}}\right): \boldsymbol{\phi} \\
& \left.-2\left((\boldsymbol{\nabla} \boldsymbol{w}) \beta_{\delta}(\boldsymbol{\psi})\right): \boldsymbol{\phi}+\frac{1}{\mathrm{Wi}}(\boldsymbol{\psi}-\boldsymbol{I}): \boldsymbol{\phi}\right]-\left\langle\boldsymbol{f}^{n}, \boldsymbol{v}\right\rangle_{H_{0}^{1}(\mathcal{D})} \\
& +\sum_{j=1}^{N_{E}} \int_{E_{j}}\left|\boldsymbol{u}_{\delta, h}^{n-1} \cdot \boldsymbol{n}\right| \llbracket \boldsymbol{\psi} \rrbracket_{\rightarrow \boldsymbol{u}_{\delta, h}^{n-1}}: \boldsymbol{\phi}^{+\boldsymbol{u}_{\delta, h}^{n-1}} \quad \forall(\boldsymbol{v}, \boldsymbol{\phi}) \in \mathrm{V}_{h}^{0} \times \mathrm{S}_{h}^{0} \cdot \quad(3.20)
\end{aligned}
$$


We note that a solution $\left(\boldsymbol{u}_{\delta, h}^{n}, \boldsymbol{\sigma}_{\delta, h}^{n}\right)$ to (3.12a b), if it exists, corresponds to a zero of $\mathcal{F}$; that is,

$$
\left(\mathcal{F}\left(\boldsymbol{u}_{\delta, h}^{n}, \boldsymbol{\sigma}_{\delta, h}^{n}\right),(\boldsymbol{v}, \boldsymbol{\phi})\right)_{\mathcal{D}}=0 \quad \forall(\boldsymbol{v}, \boldsymbol{\phi}) \in \mathrm{V}_{h}^{0} \times \mathrm{S}_{h}^{0} .
$$

In addition, it is easily deduced that the mapping $\mathcal{F}$ is continuous.

For any $(\boldsymbol{w}, \boldsymbol{\psi}) \in \mathrm{V}_{h}^{0} \times \mathrm{S}_{h}^{0}$, on choosing $(\boldsymbol{v}, \boldsymbol{\phi})=\left(\boldsymbol{w}, \frac{\varepsilon}{2 \mathrm{Wi}}\left(\boldsymbol{I}-G_{\delta}^{\prime}(\boldsymbol{\psi})\right)\right.$, we obtain analogously to (3.15) that

$$
\begin{aligned}
\left(\mathcal{F}(\boldsymbol{w}, \boldsymbol{\psi}),\left(\boldsymbol{w}, \frac{\varepsilon}{2 \mathrm{Wi}}\left(\boldsymbol{I}-G_{\delta}^{\prime}(\boldsymbol{\psi})\right)\right)\right)_{\mathcal{D}} & \\
\geq & \frac{F_{\delta}(\boldsymbol{w}, \boldsymbol{\psi})-F_{\delta}\left(\boldsymbol{u}_{\delta, h}^{n-1}, \boldsymbol{\sigma}_{\delta, h}^{n-1}\right)}{\Delta t_{n}}+\frac{\operatorname{Re}}{2 \Delta t_{n}} \int_{\mathcal{D}}\left\|\boldsymbol{w}-\boldsymbol{u}_{\delta, h}^{n-1}\right\|^{2}+\frac{1-\varepsilon}{2} \int_{\mathcal{D}}\|\boldsymbol{\nabla} \boldsymbol{w}\|^{2} \\
& \quad+\frac{\varepsilon}{2 \mathrm{Wi}^{2}} \int_{\mathcal{D}} \operatorname{tr}\left(\beta_{\delta}(\boldsymbol{\psi})+\left[\beta_{\delta}(\boldsymbol{\psi})\right]^{-1}-2 \boldsymbol{I}\right)-\frac{1+C_{P}}{2(1-\varepsilon)}\left\|\boldsymbol{f}^{n}\right\|_{H^{-1}(\mathcal{D})}^{2} \cdot(3.22)
\end{aligned}
$$

Let us now assume that for any $\gamma \in \mathbb{R}_{>0}$, the continuous mapping $\mathcal{F}$ has no zero $\left(\boldsymbol{u}_{\delta, h}^{n}, \boldsymbol{\sigma}_{\delta, h}^{n}\right)$ satisfying (3.21), which lies in the ball

$$
\mathcal{B}_{\gamma}:=\left\{(\boldsymbol{v}, \phi) \in \mathrm{V}_{h}^{0} \times \mathrm{S}_{h}^{0}:\|(\boldsymbol{v}, \phi)\|_{\mathcal{D}} \leq \gamma\right\} ;
$$

where

$$
\|(\boldsymbol{v}, \phi)\|_{D}:=\left[((\boldsymbol{v}, \phi),(\boldsymbol{v}, \phi))_{D}\right]^{\frac{1}{2}}=\left(\int_{\mathcal{D}}\left[\|\boldsymbol{v}\|^{2}+\|\phi\|^{2}\right]\right)^{\frac{1}{2}} .
$$

Then for such $\gamma$, we can define the continuous mapping $\mathcal{G}_{\gamma}: \mathcal{B}_{\gamma} \rightarrow \mathcal{B}_{\gamma}$ such that for all $(\boldsymbol{v}, \phi) \in \mathcal{B}_{\gamma}$

$$
\mathcal{G}_{\gamma}(\boldsymbol{v}, \phi):=-\gamma \frac{\mathcal{F}(\boldsymbol{v}, \phi)}{\|\mathcal{F}(\boldsymbol{v}, \phi)\|_{\mathcal{D}}} .
$$

By the Brouwer fixed point theorem, $\mathcal{G}_{\gamma}$ has at least one fixed point $\left(\boldsymbol{w}_{\gamma}, \boldsymbol{\psi}_{\gamma}\right)$ in $\mathcal{B}_{\gamma}$. Hence it satisfies

$$
\left\|\left(\boldsymbol{w}_{\gamma}, \boldsymbol{\psi}_{\gamma}\right)\right\|_{\mathcal{D}}=\left\|\mathcal{G}_{\gamma}\left(\boldsymbol{w}_{\gamma}, \boldsymbol{\psi}_{\gamma}\right)\right\|_{\mathcal{D}}=\gamma
$$

It follows, on noting (3.2c), (3.24) and (3.26), that

$$
\left\|\boldsymbol{\psi}_{\gamma}\right\|_{L^{\infty}(\mathcal{D})}^{2} \leq \frac{1}{\min _{k \in N_{K}}\left|K_{k}\right|} \int_{\mathcal{D}}\left\|\boldsymbol{\psi}_{\gamma}\right\|^{2} \equiv \mu_{h}^{2} \int_{\mathcal{D}}\left\|\boldsymbol{\psi}_{\gamma}\right\|^{2} \leq \mu_{h}^{2} \gamma^{2},
$$

where $\mu_{h}:=\left[1 /\left(\min _{k \in N_{K}}\left|K_{k}\right|\right)\right]^{\frac{1}{2}}$. It follows from (2.20), (2.8), (3.27) and (3.26) that

$$
\begin{aligned}
F_{\delta}\left(\boldsymbol{w}_{\gamma}, \boldsymbol{\psi}_{\gamma}\right) & =\frac{\operatorname{Re}}{2} \int_{\mathcal{D}}\left\|\boldsymbol{w}_{\gamma}\right\|^{2}+\frac{\varepsilon}{2 \mathrm{Wi}} \int_{\mathcal{D}} \operatorname{tr}\left(\boldsymbol{\psi}_{\gamma}-G_{\delta}\left(\boldsymbol{\psi}_{\gamma}\right)-\boldsymbol{I}\right) \\
& \geq \frac{\operatorname{Re}}{2} \int_{\mathcal{D}}\left\|\boldsymbol{w}_{\gamma}\right\|^{2}+\frac{\varepsilon}{4 \mathrm{Wi}}\left[\int_{\mathcal{D}}\left\|\boldsymbol{\psi}_{\gamma}\right\|-2 d|\mathcal{D}|\right] \\
& \geq \frac{\operatorname{Re}}{2} \int_{\mathcal{D}}\left\|\boldsymbol{w}_{\gamma}\right\|^{2}+\frac{\varepsilon}{4 \mathrm{Wi} \mu_{h} \gamma}\left\|\boldsymbol{\psi}_{\gamma}\right\|_{L^{\infty}(\mathcal{D})}\left[\int_{\mathcal{D}}\left\|\boldsymbol{\psi}_{\gamma}\right\|\right]-\frac{\varepsilon d|\mathcal{D}|}{2 \mathrm{Wi}}
\end{aligned}
$$




$$
\begin{aligned}
& \geq \min \left(\frac{\operatorname{Re}}{2}, \frac{\varepsilon}{4 \mathrm{Wi} \mu_{h} \gamma}\right)\left(\int_{\mathcal{D}}\left[\left\|\boldsymbol{w}_{\gamma}\right\|^{2}+\left\|\boldsymbol{\psi}_{\gamma}\right\|^{2}\right]\right)-\frac{\varepsilon d|\mathcal{D}|}{2 \mathrm{Wi}} \\
& =\min \left(\frac{\operatorname{Re}}{2}, \frac{\varepsilon}{4 \mathrm{Wi} \mu_{h} \gamma}\right) \gamma^{2}-\frac{\varepsilon d|\mathcal{D}|}{2 \mathrm{Wi}} .
\end{aligned}
$$

Hence for all $\gamma$ sufficiently large, it follows from (3.22) and (3.28) that

$$
\left(\mathcal{F}\left(\boldsymbol{w}_{\gamma}, \boldsymbol{\psi}_{\gamma}\right),\left(\boldsymbol{w}_{\gamma}, \frac{\varepsilon}{2 \mathrm{Wi}}\left(\boldsymbol{I}-G_{\delta}^{\prime}\left(\boldsymbol{\psi}_{\gamma}\right)\right)\right)\right)_{\mathcal{D}} \geq 0 .
$$

On the other hand as $\left(\boldsymbol{w}_{\gamma}, \boldsymbol{\psi}_{\gamma}\right)$ is a fixed point of $\mathcal{G}_{\gamma}$, we have that

$$
\begin{aligned}
& \left(\mathcal{F}\left(\boldsymbol{w}_{\gamma}, \boldsymbol{\psi}_{\gamma}\right),\left(\boldsymbol{w}_{\gamma}, \frac{\varepsilon}{2 \mathrm{Wi}}\left(\boldsymbol{I}-G_{\delta}^{\prime}\left(\boldsymbol{\psi}_{\gamma}\right)\right)\right)\right)_{D} \\
& =-\frac{\left\|\mathcal{F}\left(\boldsymbol{w}_{\gamma}, \boldsymbol{\psi}_{\gamma}\right)\right\|_{\mathcal{D}}}{\gamma} \int_{\mathcal{D}}\left[\left\|\boldsymbol{w}_{\gamma}\right\|^{2}+\frac{\varepsilon}{2 \mathrm{Wi}} \boldsymbol{\psi}_{\gamma}:\left(\boldsymbol{I}-G_{\delta}^{\prime}\left(\boldsymbol{\psi}_{\gamma}\right)\right)\right] .
\end{aligned}
$$

It follows from (2.8), and similarly to (3.28), on noting (3.27) and (3.26) that

$$
\begin{aligned}
\int_{\mathcal{D}}\left[\left\|\boldsymbol{w}_{\gamma}\right\|^{2}+\frac{\varepsilon}{2 \mathrm{Wi}} \boldsymbol{\psi}_{\gamma}:\left(\boldsymbol{I}-G_{\delta}^{\prime}\left(\boldsymbol{\psi}_{\gamma}\right)\right)\right] & \geq \int_{\mathcal{D}}\left[\left\|\boldsymbol{w}_{\gamma}\right\|^{2}+\frac{\varepsilon}{4 \mathrm{Wi}}\left[\left\|\boldsymbol{\psi}_{\gamma}\right\|-2 d\right]\right] \\
& \geq \min \left(1, \frac{\varepsilon}{4 \mathrm{Wi} \mu_{h} \gamma}\right) \gamma^{2}-\frac{\varepsilon d|\mathcal{D}|}{2 \mathrm{Wi}} .
\end{aligned}
$$

Therefore on combining (3.30) and (3.31), we have for all $\gamma$ sufficiently large that

$$
\left(\mathcal{F}\left(\boldsymbol{w}_{\gamma}, \boldsymbol{\psi}_{\gamma}\right),\left(\boldsymbol{w}_{\gamma}, \frac{\varepsilon}{2 \mathrm{Wi}}\left(\boldsymbol{I}-G_{\delta}^{\prime}\left(\boldsymbol{\psi}_{\gamma}\right)\right)\right)\right)_{D}<0,
$$

which obviously contradicts (3.29). Hence the mapping $\mathcal{F}$ has a zero in $\mathcal{B}_{\gamma}$ for $\gamma$ sufficiently large.

Theorem 3.1. For any $\delta \in\left(0, \frac{1}{2}\right], N_{T} \geq 1$ and any partitioning of $[0, T]$ into $N_{T}$ time steps, then there exists a solution $\left\{\left(\boldsymbol{u}_{\delta, h}^{n}, \boldsymbol{\sigma}_{\delta, h}^{n}\right)\right\}_{n=1}^{N_{T}} \in\left[\mathrm{V}_{h}^{0} \times \mathrm{S}_{h}^{0}\right]^{N_{T}}$ to $\left(P_{\delta, h}^{\Delta t}\right)$.

In addition, it follows for $n=1, \ldots, N_{T}$ that

$$
\begin{gathered}
F_{\delta}\left(\boldsymbol{u}_{\delta, h}^{n}, \boldsymbol{\sigma}_{\delta, h}^{n}\right)+\frac{1}{2} \sum_{m=1}^{n} \int_{\mathcal{D}}\left[\operatorname{Re}\left\|\boldsymbol{u}_{\delta, h}^{m}-\boldsymbol{u}_{\delta, h}^{m-1}\right\|^{2}+(1-\varepsilon) \Delta t_{m}\left\|\boldsymbol{\nabla} \boldsymbol{u}_{\delta, h}^{m}\right\|^{2}\right] \\
+\frac{\varepsilon}{2 \mathrm{Wi}^{2}} \sum_{m=1}^{n} \Delta t_{m} \int_{\mathcal{D}} \operatorname{tr}\left(\beta_{\delta}\left(\boldsymbol{\sigma}_{\delta, h}^{m}\right)+\left[\beta_{\delta}\left(\boldsymbol{\sigma}_{\delta, h}^{m}\right)\right]^{-1}-2 \boldsymbol{I}\right) \\
\leq F_{\delta}\left(\boldsymbol{u}_{h}^{0}, \boldsymbol{\sigma}_{h}^{0}\right)+\frac{1+C_{P}}{2(1-\varepsilon)} \sum_{m=1}^{n} \Delta t_{m}\left\|\boldsymbol{f}^{m}\right\|_{H^{-1}(\mathcal{D})}^{2} \leq C .
\end{gathered}
$$

Moreover, it follows that

$$
\max _{n=0, \ldots, N_{T}} \int_{\mathcal{D}}\left[\left\|\boldsymbol{u}_{\delta, h}^{n}\right\|^{2}+\left\|\boldsymbol{\sigma}_{\delta, h}^{n}\right\|+\delta^{-1}\left\|\left[\boldsymbol{\sigma}_{\delta, h}^{n}\right]-\right\|\right]+\sum_{n=1}^{N_{T}} \Delta t_{n} \int_{\mathcal{D}}\left\|\left[\beta_{\delta}\left(\boldsymbol{\sigma}_{\delta, h}^{n}\right)\right]^{-1}\right\| \leq C .
$$


Proof. Existence and the stability result (3.33) follow immediately from Propositions 3.2 and 3.1, respectively, on noting (2.20), (3.11b), 3.10a) and (2.19). The bounds (3.34) follow immediately from (3.33), on noting (2.7b), (2.8), (1.7) and the fact that $\beta_{\delta}(\phi) \in \mathbb{R}_{S P D}^{d \times d}$ for any $\phi \in \mathbb{R}_{S}^{d \times d}$.

\subsection{Convergence of $\left(P_{\delta, h}^{\Delta t}\right)$ to $\left(P_{h}^{\Delta t}\right)$}

We now consider the corresponding direct finite element approximation of (P), i.e. $\left(\mathrm{P}_{\delta, h}^{\Delta t}\right)$ without the regularization $\delta$ :

$\left(\mathbf{P}_{h}^{\Delta t}\right)$ Given initial conditions $\left(\boldsymbol{u}_{h}^{0}, \boldsymbol{\sigma}_{h}^{0}\right) \in \mathrm{V}_{h}^{0} \times \mathrm{S}_{h}^{0}$ with $\boldsymbol{\sigma}_{h}^{0}$ satisfying (3.11a, b), then for $n=1, \ldots, N_{T}$ find $\left(\boldsymbol{u}_{h}^{n}, \boldsymbol{\sigma}_{h}^{n}\right) \in \mathrm{V}_{h}^{0} \times \mathrm{S}_{h}^{0}$ such that for any test functions $(\boldsymbol{v}, \phi) \in \mathrm{V}_{h}^{0} \times \mathrm{S}_{h}^{0}$

$$
\begin{gathered}
\int_{\mathcal{D}}\left[\operatorname{Re}\left(\frac{\boldsymbol{u}_{h}^{n}-\boldsymbol{u}_{h}^{n-1}}{\Delta t_{n}}\right) \cdot \boldsymbol{v}+\frac{\operatorname{Re}}{2}\left[\left(\left(\boldsymbol{u}_{h}^{n-1} \cdot \nabla\right) \boldsymbol{u}_{h}^{n}\right) \cdot \boldsymbol{v}-\boldsymbol{u}_{h}^{n} \cdot\left(\left(\boldsymbol{u}_{h}^{n-1} \cdot \nabla\right) \boldsymbol{v}\right)\right]\right. \\
\left.+(1-\varepsilon) \boldsymbol{\nabla} \boldsymbol{u}_{h}^{n}: \boldsymbol{\nabla} \boldsymbol{v}+\frac{\varepsilon}{\mathrm{Wi}} \boldsymbol{\sigma}_{h}^{n}: \boldsymbol{\nabla} \boldsymbol{v}\right]=\left\langle\boldsymbol{f}^{n}, \boldsymbol{v}\right\rangle_{H_{0}^{1}(\mathcal{D})}, \\
\int_{\mathcal{D}}\left[\left(\frac{\boldsymbol{\sigma}_{h}^{n}-\boldsymbol{\sigma}_{h}^{n-1}}{\Delta t_{n}}\right): \boldsymbol{\phi}-2\left(\left(\boldsymbol{\nabla} \boldsymbol{u}_{h}^{n}\right) \boldsymbol{\sigma}_{h}^{n}\right): \boldsymbol{\phi}+\frac{1}{\mathrm{Wi}}\left(\boldsymbol{\sigma}_{h}^{n}-\boldsymbol{I}\right): \boldsymbol{\phi}\right] \\
+\sum_{j=1}^{N_{E}} \int_{E_{j}}\left|\boldsymbol{u}_{h}^{n-1} \cdot \boldsymbol{n}\right| \llbracket \boldsymbol{\sigma}_{h}^{n} \rrbracket_{\rightarrow \boldsymbol{u}_{h}^{n-1}}: \boldsymbol{\phi}^{+\boldsymbol{u}_{h}^{n-1}}=0
\end{gathered}
$$

We introduce also the unregularised free energy

$$
F(\boldsymbol{v}, \phi):=\frac{\operatorname{Re}}{2} \int_{\mathcal{D}}\|\boldsymbol{v}\|^{2}+\frac{\varepsilon}{2 \mathrm{Wi}} \int_{\mathcal{D}} \operatorname{tr}(\boldsymbol{\phi}-G(\boldsymbol{\phi})-\boldsymbol{I}),
$$

which is well defined for $(\boldsymbol{v}, \phi) \in \mathrm{V}_{h}^{0} \times \mathrm{S}_{h}^{0}$ with $\boldsymbol{\phi}$ being positive definite on $\mathcal{D}$.

Theorem 3.2. For all regular partitionings $\mathcal{T}_{h}$ of $\mathcal{D}$ into simplices $\left\{K_{k}\right\}_{k=1}^{N_{K}}$ and all partitionings $\left\{\Delta t_{n}\right\}_{n=1}^{N_{T}}$ of $[0, T]$, there exists a subsequence $\left\{\left\{\left(\boldsymbol{u}_{\delta, h}^{n}, \boldsymbol{\sigma}_{\delta, h}^{n}\right)\right\}_{n=1}^{N_{T}}\right\}_{\delta>0}$, where $\left\{\left(\boldsymbol{u}_{\delta, h}^{n}, \boldsymbol{\sigma}_{\delta, h}^{n}\right)\right\}_{n=1}^{N_{T}} \in\left[\mathrm{V}_{h}^{0} \times \mathrm{S}_{h}^{0}\right]^{N_{T}}$ solves $\left(P_{\delta, h}^{\Delta t}\right)$, and $\left\{\left(\boldsymbol{u}_{h}^{n}, \boldsymbol{\sigma}_{h}^{n}\right)\right\}_{n=1}^{N_{T}} \in\left[\mathrm{V}_{h}^{0} \times\right.$ $\left.\mathrm{S}_{h}^{0}\right]^{N_{T}}$ such that for the subsequence

$$
\boldsymbol{u}_{\delta, h}^{n} \rightarrow \boldsymbol{u}_{h}^{n}, \quad \boldsymbol{\sigma}_{\delta, h}^{n} \rightarrow \boldsymbol{\sigma}_{h}^{n} \quad \text { as } \delta \rightarrow 0_{+}, \quad \text { for } \quad n=1, \ldots, N_{T} .
$$

In addition, for $n=1, \ldots, N_{T},\left.\boldsymbol{\sigma}_{h}^{n}\right|_{K_{k}} \in \mathbb{R}_{S P D}^{d \times d}, k=1, \ldots, N_{K}, \quad$. Moreover, $\left\{\left(\boldsymbol{u}_{h}^{n}, \boldsymbol{\sigma}_{h}^{n}\right)\right\}_{n=1}^{N_{T}} \in\left[\mathrm{V}_{h}^{0} \times \mathrm{S}_{h}^{0}\right]^{N_{T}}$ solves $\left(P_{h}^{\Delta t}\right)$ and for $n=1, \ldots, N_{T}$

$$
\begin{gathered}
\frac{F\left(\boldsymbol{u}_{h}^{n}, \boldsymbol{\sigma}_{h}^{n}\right)-F\left(\boldsymbol{u}_{h}^{n-1}, \boldsymbol{\sigma}_{h}^{n-1}\right)}{\Delta t_{n}}+\frac{\operatorname{Re}}{2 \Delta t_{n}} \int_{\mathcal{D}}\left\|\boldsymbol{u}_{h}^{n}-\boldsymbol{u}_{h}^{n-1}\right\|^{2}+(1-\varepsilon) \int_{\mathcal{D}}\left\|\boldsymbol{\nabla} \boldsymbol{u}_{h}^{n}\right\|^{2} \\
\quad+\frac{\varepsilon}{2 \mathrm{Wi}^{2}} \int_{\mathcal{D}} \operatorname{tr}\left(\boldsymbol{\sigma}_{h}^{n}+\left[\boldsymbol{\sigma}_{h}^{n}\right]^{-1}-2 \boldsymbol{I}\right) \\
\leq \frac{1}{2}(1-\varepsilon) \int_{\mathcal{D}}\left\|\boldsymbol{\nabla} \boldsymbol{u}_{h}^{n}\right\|^{2}+\frac{1+C_{P}}{2(1-\varepsilon)}\left\|\boldsymbol{f}^{n}\right\|_{H^{-1}(\mathcal{D})}^{2}
\end{gathered}
$$


Proof. For any integer $n \in\left[1, N_{T}\right]$, the desired subsequence convergence result (3.37) follows immediately from (3.34), as $\left(\boldsymbol{u}_{\delta, h}^{n}, \boldsymbol{\sigma}_{\delta, h}^{n}\right)$ are finite dimensional for fixed $\mathrm{V}_{h}^{0} \times S_{h}^{0}$. It also follows from (3.34), (3.37) and (2.17) that $\left[\boldsymbol{\sigma}_{h}^{n}\right]_{-}$vanishes on $\mathcal{D}$, so that $\boldsymbol{\sigma}_{h}^{n}$ must be non-negative definite on $\mathcal{D}$. Hence on noting this, (2.17) and (3.37), we have the following subsequence convergence results

$$
\beta_{\delta}\left(\boldsymbol{\sigma}_{h}^{n}\right) \rightarrow \boldsymbol{\sigma}_{h}^{n} \quad \text { as } \quad \delta \rightarrow 0_{+} \quad \text { and } \quad \beta_{\delta}\left(\boldsymbol{\sigma}_{\delta, h}^{n}\right) \rightarrow \boldsymbol{\sigma}_{h}^{n} \quad \text { as } \quad \delta \rightarrow 0_{+} .
$$

It also follows from (3.34), (3.39) and as $\left[\beta_{\delta}\left(\boldsymbol{\sigma}_{\delta, h}^{n}\right)\right]^{-1} \beta_{\delta}\left(\boldsymbol{\sigma}_{\delta, h}^{n}\right)=\boldsymbol{I}$ that the following subsequence result

$$
\left[\beta_{\delta}\left(\boldsymbol{\sigma}_{\delta, h}^{n}\right)\right]^{-1} \rightarrow\left[\boldsymbol{\sigma}_{h}^{n}\right]^{-1} \quad \text { as } \quad \delta \rightarrow 0_{+}
$$

holds, and so $\boldsymbol{\sigma}_{h}^{n}$ is positive definite on $\mathcal{D}$. Therefore, we have from (3.37) and (2.1) that

$$
G_{\delta}\left(\boldsymbol{\sigma}_{\delta, h}^{n}\right) \rightarrow G\left(\boldsymbol{\sigma}_{h}^{n}\right) \quad \text { as } \quad \delta \rightarrow 0_{+} .
$$

Since $\boldsymbol{u}_{\delta, h}^{n-1}, \boldsymbol{u}_{h}^{n-1} \in C(\overline{\mathcal{D}})$, it follows from the $\mathrm{S}_{h}^{0}$ version of (3.8), (3.6) and (3.37) that for $j=1, \ldots, N_{E}$ and for all $\phi \in \mathrm{S}_{h}^{0}$

$$
\begin{gathered}
\sum_{j=1}^{N_{E}} \int_{E_{j}}\left|\boldsymbol{u}_{\delta, h}^{n-1} \cdot \boldsymbol{n}\right| \llbracket \boldsymbol{\sigma}_{\delta, h}^{n} \rrbracket_{\rightarrow \boldsymbol{u}_{\delta, h}^{n-1}}: \boldsymbol{\phi}^{+\boldsymbol{u}_{\delta, h}^{n-1}}=-\sum_{k=1}^{N_{K}} \int_{\partial K_{k}}\left(\boldsymbol{u}_{\delta, h}^{n-1} \cdot \boldsymbol{n}_{K_{k}}\right) \boldsymbol{\sigma}_{\delta, h}^{n}: \boldsymbol{\phi}^{+\boldsymbol{u}_{\delta, h}^{n-1}} \\
\rightarrow-\sum_{k=1}^{N_{K}} \int_{\partial K_{k}}\left(\boldsymbol{u}_{h}^{n-1} \cdot \boldsymbol{n}_{K_{k}}\right) \boldsymbol{\sigma}_{h}^{n}: \boldsymbol{\phi}^{+\boldsymbol{u}_{h}^{n-1}}=\sum_{j=1}^{N_{E}} \int_{E_{j}}\left|\boldsymbol{u}_{h}^{n-1} \cdot \boldsymbol{n}\right| \llbracket \boldsymbol{\sigma}_{h}^{n} \rrbracket_{\rightarrow \boldsymbol{u}_{h}^{n-1}}: \boldsymbol{\phi}^{+\boldsymbol{u}_{h}^{n-1}} \\
\text { as } \quad \delta \rightarrow 0_{+} \cdot
\end{gathered}
$$

Hence using (3.37), (3.39) and (3.42), we can pass to the limit $\delta \rightarrow 0_{+}$in $\left(\mathrm{P}_{\delta, h}^{\Delta t}\right)$, (3.12a,b), to show that $\left\{\left(\boldsymbol{u}_{h}^{n}, \boldsymbol{\sigma}_{h}^{n}\right)\right\}_{n=1}^{N_{T}} \in\left[\mathrm{V}_{h}^{0} \times \mathrm{S}_{h}^{0}\right]^{N_{T}}$ solves $\left(\mathrm{P}_{h}^{\Delta t}\right)$, (3.35a,b). Similarly, using (3.37), (3.39), (3.40) and (3.41), and noting (2.20) and (3.36), we can pass to the limit $\delta \rightarrow 0_{+}$in (3.15) to obtain the desired result (3.38).

Remark 3.1. Most numerical approximations of $(\mathrm{P})$ suffer from instabilities when Wi is relatively large, the so-called high Weissenberg number problem (HWNP). This problem is still not fully understood. Some reasons for these instabilities are discussed in Boyaval et al., BLM09 e.g. poor numerical scheme or the lack of existence of a solution to (P) itself. In addition in Boyaval et al. BLM09 $\mathrm{fi}$ nite element approximations of $(\mathrm{P})$ such as $\left(\mathrm{P}_{h}^{\Delta t}\right)$, approximating the primitive variables $(\boldsymbol{u}, p, \boldsymbol{\sigma})$, are compared with finite element approximations of the logformulation of $(\mathrm{P})$, introduced in Fattal and Kupferman, FK05 which is based on the variables $(\boldsymbol{u}, p, \boldsymbol{\psi})$, where $\boldsymbol{\psi}=\ln \boldsymbol{\sigma}$. The equivalent free energy estimate for this log-formulation is based on testing the Navier-Stokes equation with $\boldsymbol{u}$ as before, but the $\log$-form of the stress equation with $(\exp \boldsymbol{\psi}-\boldsymbol{I})$. Whereas the free energy estimate for (P) requires $\boldsymbol{\sigma}$ to be positive definite, due to the testing with $\ln \boldsymbol{\sigma}$, the free energy estimate for the log-formulation requires no such constraint. In Boyaval et al.BLM09 a constraint, based on the initial data, was required on the time 
step in order to ensure that the approximation to $\sigma$ remained positive definite for schemes such as $\left(\mathrm{P}_{h}^{\Delta t}\right)$ approximating $(\mathrm{P})$; whereas existence of a solution to finite element approximations of the log-formulation, and satisfying a discrete log-form of the free energy estimate, were shown for any choice of time step. It was suggested

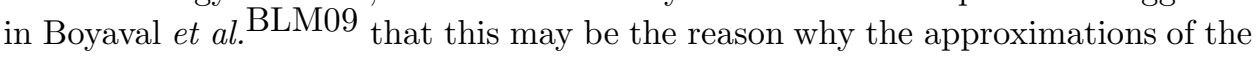
log-formulation are reported to be more stable than those based on $(\mathrm{P})$. However, Theorem 3.2 above shows that there does exist (at least) one solution to $\left(\mathrm{P}_{h}^{\Delta t}\right)$, which satisfies the free energy estimate (3.38), whatever the time step. Of course, we do not have a uniqueness proof for $\left(\mathrm{P}_{h}^{\Delta t}\right)$.

\section{Regularized problems with stress diffusion and possibly the cut-off $\beta^{L}$}

\subsection{Regularizations, $\left(P_{\alpha}^{(L)}\right)$, of $(P)$ with stress diffusion and possibly the cut-off $\beta^{L}$}

In this section, we consider the following modified versions of $(\mathrm{P})$ for given constants $\alpha \in \mathbb{R}_{>0}$ and $L \geq 2$ :

$\left(\mathbf{P}_{\alpha}^{(L)}\right)$ Find $\boldsymbol{u}_{\alpha}^{(L)}:(t, \boldsymbol{x}) \in[0, T) \times \mathcal{D} \mapsto \boldsymbol{u}_{\alpha}^{(L)}(t, \boldsymbol{x}) \in \mathbb{R}^{d}, p_{\alpha}^{(L)}:(t, \boldsymbol{x}) \in(0, T) \times \mathcal{D} \mapsto$ $p_{\alpha}^{(L)}(t, \boldsymbol{x}) \in \mathbb{R}$ and $\boldsymbol{\sigma}_{\alpha}^{(L)}:(t, \boldsymbol{x}) \in[0, T) \times \mathcal{D} \mapsto \boldsymbol{\sigma}_{\alpha}^{(L)}(t, \boldsymbol{x}) \in \mathbb{R}_{S}^{d \times d}$ such that

$$
\begin{aligned}
& \operatorname{Re}\left(\frac{\partial \boldsymbol{u}_{\alpha}^{(L)}}{\partial t}+\left(\boldsymbol{u}_{\alpha}^{(L)} \cdot \nabla\right) \boldsymbol{u}_{\alpha}^{(L)}\right)=-\nabla p_{\alpha}^{(L)}+(1-\varepsilon) \Delta \boldsymbol{u}_{\alpha}^{(L)}+\frac{\varepsilon}{\mathrm{Wi}} \operatorname{div} \beta^{(L)}\left(\boldsymbol{\sigma}_{\alpha}^{(L)}\right) \\
& +\boldsymbol{f} \quad \text { on } \mathcal{D}_{T}, \\
& \operatorname{div} \boldsymbol{u}_{\alpha}^{(L)}=0 \quad \text { on } \mathcal{D}_{T}, \\
& \frac{\partial \boldsymbol{\sigma}_{\alpha}^{(L)}}{\partial t}+\left(\boldsymbol{u}_{\alpha}^{(L)} \cdot \boldsymbol{\nabla}\right) \beta^{(L)}\left(\boldsymbol{\sigma}_{\alpha}^{(L)}\right)=\left(\boldsymbol{\nabla} \boldsymbol{u}_{\alpha}^{(L)}\right) \beta^{(L)}\left(\boldsymbol{\sigma}_{\alpha}^{(L)}\right)+\beta^{(L)}\left(\boldsymbol{\sigma}_{\alpha}^{(L)}\right)\left(\boldsymbol{\nabla} \boldsymbol{u}_{\alpha}^{(L)}\right)^{T} \\
& -\frac{1}{\mathrm{Wi}}\left(\boldsymbol{\sigma}_{\alpha}^{(L)}-\boldsymbol{I}\right)+\alpha \Delta \boldsymbol{\sigma}_{\alpha}^{(L)} \quad \text { on } \mathcal{D}_{T} \\
& \boldsymbol{u}_{\alpha}^{(L)}(0, \boldsymbol{x})=\boldsymbol{u}^{0}(\boldsymbol{x}) \quad \forall \boldsymbol{x} \in \mathcal{D}, \\
& \boldsymbol{\sigma}_{\alpha}^{(L)}(0, \boldsymbol{x})=\boldsymbol{\sigma}^{0}(\boldsymbol{x}) \quad \forall \boldsymbol{x} \in \mathcal{D}, \\
& \boldsymbol{u}_{\alpha}^{(L)}=\mathbf{0} \quad \text { on }(0, T) \times \partial \mathcal{D}, \\
& \left(\boldsymbol{n}_{\partial \mathcal{D}} \cdot \boldsymbol{\nabla}\right) \boldsymbol{\sigma}_{\alpha}^{(L)}=\mathbf{0} \quad \text { on }(0, T) \times \partial \mathcal{D} ;
\end{aligned}
$$

where $\boldsymbol{n}_{\mathcal{D}}$ is normal to the boundary $\partial \mathcal{D}$.

Hence problem $\left(\mathrm{P}_{\alpha}^{(L)}\right)$ is the same as $(\mathrm{P})$, but with the added diffusion term $\alpha \Delta \boldsymbol{\sigma}_{\alpha}^{(L)}$ for the stress equation (4.1C), and the associated Neumann boundary condition 4.1g); and in the case of $\left(\mathrm{P}_{\alpha}^{L}\right)$ with certain terms in (4.1a, c) involving $\boldsymbol{\sigma}_{\alpha}^{L}$ replaced by $\beta^{L}\left(\boldsymbol{\sigma}_{\alpha}^{L}\right)$, recall (2.3). Of course, it is naturally assumed in $\left(\mathrm{P}_{\alpha}^{(L)}\right)$ that $\boldsymbol{\sigma}_{\alpha}^{(L)}$ is positive definite on $\mathcal{D}_{T}$ in order for $\beta^{(L)}\left(\boldsymbol{\sigma}_{\alpha}^{(L)}\right)$ to be well defined.

We will also be interested in the corresponding regularization $\left(\mathrm{P}_{\alpha, \delta}^{(L)}\right)$ of $\left(\mathrm{P}_{\alpha}^{(L)}\right)$ with solution $\left(\boldsymbol{u}_{\alpha, \delta}^{(L)}, p_{\alpha, \delta}^{(L)}, \boldsymbol{\sigma}_{\alpha, \delta}^{(L)}\right)$; where $\beta^{(L)}(\cdot)$ in (4.1a-g) is replaced by $\beta_{\delta}^{(L)}(\cdot)$, and 
so that $\sigma_{\alpha, \delta}^{(L)}$ is not required to be positive definite.

\subsection{Formal energy estimates for $\left(P_{\alpha, \delta}^{(L)}\right)$}

Let $F_{\delta}^{(L)}\left(\boldsymbol{u}_{\alpha, \delta}^{(L)}, \boldsymbol{\sigma}_{\alpha, \delta}^{(L)}\right)$ denote the free energy of the solution $\left(\boldsymbol{u}_{\alpha, \delta}^{(L)}, p_{\alpha, \delta}^{(L)}, \boldsymbol{\sigma}_{\alpha, \delta}^{(L)}\right)$ to problem $\left(\mathrm{P}_{\alpha, \delta}^{(\mathrm{L})}\right)$, where $F_{\delta}^{(L)}: \mathrm{W} \times \mathrm{S} \rightarrow \mathbb{R}$ is defined as

$$
F_{\delta}^{(L)}(\boldsymbol{v}, \phi):=\frac{\operatorname{Re}}{2} \int_{\mathcal{D}}\|\boldsymbol{v}\|^{2}+\frac{\varepsilon}{2 \mathrm{Wi}} \int_{\mathcal{D}} \operatorname{tr}\left(\boldsymbol{\phi}-G_{\delta}^{(L)}(\phi)-\boldsymbol{I}\right) .
$$

We have the following analogue of Proposition 2.1.

Proposition 4.1. Let $\left(\boldsymbol{u}_{\alpha, \delta}^{(L)}, p_{\alpha, \delta}^{(L)}, \boldsymbol{\sigma}_{\alpha, \delta}^{(L)}\right)$ be a sufficiently smooth solution to problem $\left(\mathrm{P}_{\alpha, \delta}^{(\mathrm{L})}\right)$. Then the free energy $F_{\delta}^{(L)}\left(\boldsymbol{u}_{\alpha, \delta}^{(L)}, \boldsymbol{\sigma}_{\alpha, \delta}^{(L)}\right)$ satisfies for a.a. $t \in(0, T)$

$$
\begin{aligned}
& \frac{d}{d t} F_{\delta}^{(L)}\left(\boldsymbol{u}_{\alpha, \delta}^{(L)}, \boldsymbol{\sigma}_{\alpha, \delta}^{(L)}\right)+(1-\varepsilon) \int_{\mathcal{D}}\left\|\boldsymbol{\nabla} \boldsymbol{u}_{\alpha, \delta}^{(L)}\right\|^{2} \\
& +\frac{\varepsilon}{2 \mathrm{Wi}^{2}} \int_{\mathcal{D}} \operatorname{tr}\left(\beta_{\delta}^{(L)}\left(\boldsymbol{\sigma}_{\alpha, \delta}^{(L)}\right)+\left[\beta_{\delta}^{(L)}\left(\boldsymbol{\sigma}_{\alpha, \delta}^{(L)}\right)\right]^{-1}-2 \boldsymbol{I}\right) \\
& \quad+\frac{\alpha \varepsilon \delta^{2}}{2 \mathrm{Wi}} \int_{\mathcal{D}}\left\|\boldsymbol{\nabla} G_{\delta}^{(L)^{\prime}}\left(\boldsymbol{\sigma}_{\alpha, \delta}^{(L)}\right)\right\|^{2} \leq\left\langle\boldsymbol{f}, \boldsymbol{u}_{\alpha, \delta}^{(L)}\right\rangle_{H_{0}^{1}(\mathcal{D})} .
\end{aligned}
$$

Proof. Multiplying the Navier-Stokes equation (4.1a) with $\boldsymbol{u}_{\alpha, \delta}^{(L)}$ and the stress equation (4.1C) with $\frac{\varepsilon}{2 \mathrm{Wi}}\left(\boldsymbol{I}-G_{\delta}^{(L)^{\prime}}\left(\boldsymbol{\sigma}_{\alpha, \delta}^{(L)}\right)\right)$, summing and integrating over $\mathcal{D}$ yields, after using integrations by parts and the incompressibility property in the standard way, that

$$
\begin{aligned}
\int_{\mathcal{D}} & {\left[\frac{\operatorname{Re}}{2} \frac{\partial}{\partial t}\left\|\boldsymbol{u}_{\alpha, \delta}^{(L)}\right\|^{2}+(1-\varepsilon)\left\|\nabla \boldsymbol{u}_{\alpha, \delta}^{(L)}\right\|^{2}\right] } \\
+ & \frac{\varepsilon}{\mathrm{Wi}} \int_{\mathcal{D}}\left[\beta_{\delta}^{(L)}\left(\boldsymbol{\sigma}_{\alpha, \delta}^{(L)}\right): \nabla \boldsymbol{u}_{\alpha, \delta}^{(L)}-\frac{\alpha}{2} \boldsymbol{\nabla} \boldsymbol{\sigma}_{\alpha, \delta}^{(L)}:: \nabla G_{\delta}^{(L)^{\prime}}\left(\boldsymbol{\sigma}_{\alpha, \delta}^{(L)}\right)\right. \\
+ & \frac{1}{2}\left(\left(\frac{\partial}{\partial t} \boldsymbol{\sigma}_{\alpha, \delta}^{(L)}+\left(\boldsymbol{u}_{\alpha, \delta}^{(L)} \cdot \boldsymbol{\nabla}\right) \beta_{\delta}^{(L)}\left(\boldsymbol{\sigma}_{\alpha, \delta}^{(L)}\right)\right)+\frac{1}{\mathrm{Wi}}\left(\boldsymbol{\sigma}_{\alpha, \delta}^{(L)}-\boldsymbol{I}\right)\right):\left(\boldsymbol{I}-G_{\delta}^{(L)^{\prime}}\left(\boldsymbol{\sigma}_{\alpha, \delta}^{(L)}\right)\right) \\
- & \frac{1}{2}\left(\left(\boldsymbol{\nabla} \boldsymbol{u}_{\alpha, \delta}^{(L)}\right) \beta_{\delta}^{(L)}\left(\boldsymbol{\sigma}_{\alpha, \delta}^{(L)}\right)+\beta_{\delta}^{(L)}\left(\boldsymbol{\sigma}_{\alpha, \delta}^{(L)}\right)\left(\boldsymbol{\nabla} \boldsymbol{u}_{\alpha, \delta}^{(L)}\right)^{T}\right):\left(\boldsymbol{I}-G_{\delta}^{(L)^{\prime}}\left(\boldsymbol{\sigma}_{\alpha, \delta}^{(L)}\right)\right] \\
& =\left\langle\boldsymbol{f}, \boldsymbol{u}_{\alpha, \delta}^{(L)}\right\rangle_{H_{0}^{1}(\mathcal{D})} .
\end{aligned}
$$

Similarly to (2.12), we have that

$$
-\nabla \sigma_{\alpha, \delta}^{(L)}:: \nabla G_{\delta}^{(L)^{\prime}}\left(\boldsymbol{\sigma}_{\alpha, \delta}^{(L)}\right) \geq \delta^{2}\left\|\nabla G_{\delta}^{(L)^{\prime}}\left(\boldsymbol{\sigma}_{\alpha, \delta}^{(L)}\right)\right\|^{2} \quad \text { a.e. in } \mathcal{D}_{T} .
$$

Using (2.16), we have that

$$
\frac{\partial}{\partial t} \boldsymbol{\sigma}_{\alpha, \delta}^{(L)}:\left(\boldsymbol{I}-G_{\delta}^{(L)^{\prime}}\left(\boldsymbol{\sigma}_{\alpha, \delta}^{(L)}\right)\right)=\frac{\partial}{\partial t} \operatorname{tr}\left(\boldsymbol{\sigma}_{\alpha, \delta}^{(L)}-G_{\delta}^{(L)}\left(\boldsymbol{\sigma}_{\alpha, \delta}^{(L)}\right)\right) .
$$


We will deal with the convection term differently to the approach used in (2.23), as that cannot be mimicked at a discrete level using continuous piecewise linear elements to approximate $\boldsymbol{\sigma}_{\alpha, \delta}^{(L)}$. Note that we cannot use $S_{h}^{0}$ with the desirable property (3.5) to approximate $\boldsymbol{\sigma}_{\alpha, \delta}^{(L)}$, as we now have the added diffusion term. Instead, as $\boldsymbol{\sigma}_{\alpha, \delta}^{(L)}$ has been replaced by $\beta_{\delta}^{(L)}\left(\boldsymbol{\sigma}_{\alpha, \delta}^{(L)}\right) \equiv H_{\delta}^{(L)^{\prime}}\left(G_{\delta}^{(L)^{\prime}}\left(\boldsymbol{\sigma}_{\alpha, \delta}^{(L)}\right)\right)$, on recalling (2.6), in this convective term and as $\boldsymbol{u}_{\alpha, \delta}^{(L)} \in \mathrm{V}$, we have that

$$
\begin{aligned}
\int_{\mathcal{D}}\left(\boldsymbol{u}_{\alpha, \delta}^{(L)} \cdot \nabla\right) \beta_{\delta}^{(L)}\left(\boldsymbol{\sigma}_{\alpha, \delta}^{(L)}\right):(\boldsymbol{I} & \left.-G_{\delta}^{(L)^{\prime}}\left(\boldsymbol{\sigma}_{\alpha, \delta}^{(L)}\right)\right) \\
& =\int_{\mathcal{D}} \beta_{\delta}^{(L)}\left(\boldsymbol{\sigma}_{\alpha, \delta}^{(L)}\right):\left(\boldsymbol{u}_{\alpha, \delta}^{(L)} \cdot \nabla\right) G_{\delta}^{(L)^{\prime}}\left(\boldsymbol{\sigma}_{\alpha, \delta}^{(L)}\right) \\
& =\int_{\mathcal{D}}\left(\boldsymbol{u}_{\alpha, \delta}^{(L)} \cdot \boldsymbol{\nabla}\right) \operatorname{tr}\left(H_{\delta}^{(L)}\left(G_{\delta}^{(L)^{\prime}}\left(\boldsymbol{\sigma}_{\alpha, \delta}^{(L)}\right)\right)\right)=0,
\end{aligned}
$$

where we have noted the spatial counterpart of (2.16). Similarly to (2.24) and (2.25) we obtain that

$$
\begin{aligned}
&\left(\left(\boldsymbol{\nabla} \boldsymbol{u}_{\alpha, \delta}^{(L)}\right) \beta_{\delta}^{(L)}\left(\boldsymbol{\sigma}_{\alpha, \delta}^{(L)}\right)+\beta_{\delta}^{(L)}\left(\boldsymbol{\sigma}_{\alpha, \delta}^{(L)}\right)\left(\boldsymbol{\nabla} \boldsymbol{u}_{\alpha, \delta}^{(L)}\right)^{T}\right):\left(\boldsymbol{I}-G_{\delta}^{(L)^{\prime}}\left(\boldsymbol{\sigma}_{\alpha, \delta}^{(L)}\right)\right) \\
&=2 \operatorname{tr}\left(\left(\boldsymbol{\nabla} \boldsymbol{u}_{\alpha, \delta}^{(L)}\right) \beta_{\delta}^{(L)}\left(\boldsymbol{\sigma}_{\alpha, \delta}^{(L)}\right)\right)
\end{aligned}
$$

and once again the terms involving the left-hand side of (4.8) in (4.4) cancel with the term $\frac{\varepsilon}{\mathrm{Wi}} \beta_{\delta}^{(L)}\left(\boldsymbol{\sigma}_{\alpha, \delta}^{(L)}\right): \nabla \boldsymbol{u}_{\alpha, \delta}^{(L)}$ in (4.4) arising from the Navier-Stokes equation.

Finally, the treatment of the remaining term $\left(\boldsymbol{\sigma}_{\alpha, \delta}^{(L)}-\boldsymbol{I}\right):\left(\boldsymbol{I}-G_{\delta}^{(L)^{\prime}}\left(\boldsymbol{\sigma}_{\alpha, \delta}^{(L)}\right)\right)$ follows similarly to (2.26); and so we obtain the desired free energy inequality (4.3).

The following Corollary follows from (4.3) on noting the proof of Corollary 2.1.

Corollary 4.1. Let $\left(\boldsymbol{u}_{\alpha, \delta}^{(L)}, p_{\alpha, \delta}^{(L)}, \boldsymbol{\sigma}_{\alpha, \delta}^{(L)}\right)$ be a sufficiently smooth solution to problem $\left(\mathrm{P}_{\alpha, \delta}^{(\mathrm{L})}\right)$. Then it follows that

$$
\begin{aligned}
\sup _{t \in(0, T)} F_{\delta}^{(L)}\left(\boldsymbol{u}_{\alpha, \delta}^{(L)}(t, \cdot),\right. & \left.\boldsymbol{\sigma}_{\alpha, \delta}^{(L)}(t, \cdot)\right)+\frac{\alpha \varepsilon \delta^{2}}{2 \mathrm{Wi}} \int_{D_{T}}\left\|\boldsymbol{\nabla} G_{\delta}^{(L)}{ }^{\prime}\left(\boldsymbol{\sigma}_{\alpha, \delta}^{(L)}\right)\right\|^{2} \\
+\frac{1}{2} \int_{\mathcal{D}_{T}}[(1-\varepsilon) \| & \left.\boldsymbol{\nabla} \boldsymbol{u}_{\alpha, \delta}^{(L)} \|^{2}+\frac{\varepsilon}{\mathrm{Wi}^{2}} \operatorname{tr}\left(\beta_{\delta}^{(L)}\left(\boldsymbol{\sigma}_{\alpha, \delta}^{(L)}\right)+\left[\beta_{\delta}^{(L)}\left(\boldsymbol{\sigma}_{\alpha, \delta}^{(L)}\right)\right]^{-1}-2 \boldsymbol{I}\right)\right] \\
\leq & 2\left(F_{\delta}^{(L)}\left(\boldsymbol{u}^{0}, \boldsymbol{\sigma}^{0}\right)+\frac{1+C_{P}}{2(1-\varepsilon)}\|\boldsymbol{f}\|_{L^{2}\left(0, T ; H^{-1}(\mathcal{D})\right)}^{2}\right) .
\end{aligned}
$$

\section{Finite element approximation of $\left(\mathbf{P}_{\alpha, \delta}^{(L)}\right)$ and $\left(\mathrm{P}_{\alpha}^{(L)}\right)$}

\subsection{Finite element discretization}

We now introduce a conforming finite element discretization of $\left(\mathrm{P}_{\alpha, \delta}^{(L)}\right)$, which satisfies a discrete analogue of (4.3). As noted in the proof of Proposition 4.1 above, 
we cannot use $S_{h}^{0}$ with the desirable property (3.5) to approximate $\boldsymbol{\sigma}_{\alpha, \delta}^{(L)}$, as we now have the added diffusion term. In the following, we choose

$$
\begin{aligned}
\mathrm{W}_{h}^{1} & :=\mathrm{W}_{h}^{2} \subset \mathrm{W} \quad \text { or } \quad \mathrm{W}_{h}^{1,+} \subset \mathrm{W}, \\
\mathrm{Q}_{h}^{1} & =\left\{q \in C(\overline{\mathcal{D}}):\left.q\right|_{K_{k}} \in \mathbb{P}_{1} \quad k=1, \ldots, N_{K}\right\} \subset \mathrm{Q}, \\
\mathrm{S}_{h}^{1} & =\left\{\boldsymbol{\phi} \in[C(\overline{\mathcal{D}})]_{S}^{d \times d}:\left.\boldsymbol{\phi}\right|_{K_{k}} \in\left[\mathbb{P}_{1}\right]_{S}^{d \times d} \quad k=1, \ldots, N_{K}\right\} \subset \mathrm{S} \\
\text { and } \quad \mathrm{V}_{h}^{1} & =\left\{\boldsymbol{v} \in \mathrm{W}_{h}^{1}: \int_{\mathcal{D}} q \operatorname{div} \boldsymbol{v}=0 \quad \forall q \in \mathrm{Q}_{h}^{1}\right\} ;
\end{aligned}
$$

where $\mathrm{W}_{h}^{2}$ is defined as in (3.3a) and, on recalling the barycentric coordinate notation used in (3.4),

$$
\mathrm{W}_{h}^{1,+}:=\left\{\boldsymbol{v} \in[C(\overline{\mathcal{D}})]^{d} \cap \mathrm{W}:\left.\boldsymbol{v}\right|_{K_{k}} \in\left[\mathbb{P}_{1} \oplus \operatorname{span} \prod_{i=0}^{d} \eta_{i}^{k}\right]^{d} \quad k=1, \ldots, N_{K}\right\} .
$$

The velocity-pressure choice, $\mathrm{W}_{h}^{2} \times \mathrm{Q}_{h}^{1}$, is the lowest order Taylor-Hood element. It satisfies (3.1) with $\mathrm{W}_{h}^{0}$ and $\mathrm{Q}_{h}^{0}$ replaced by $\mathrm{W}_{h}^{2}$ and $\mathrm{Q}_{h}^{1}$, respectively, provided, in addition to $\left\{\mathcal{T}_{h}\right\}_{h>0}$ being a regular family of meshes, that each simplex has at least one vertex in $\mathcal{D}$, see p177 in Girault and Raviart GR86 in the case $d=2$ and

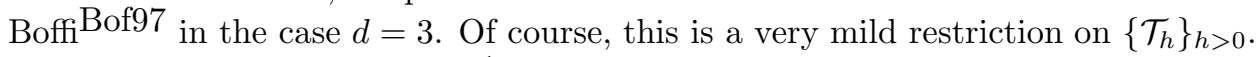
The velocity-pressure choice, $\mathrm{W}_{h}^{1,+} \times \mathrm{Q}_{h}^{1}$, is called the mini-element. It satisfies (3.1) with $\mathrm{W}_{h}^{0}$ and $\mathrm{Q}_{h}^{0}$ replaced by $\mathrm{W}_{h}^{1,+}$ and $\mathrm{Q}_{h}^{1}$, respectively; see Chapter II, Section 4.1 in Girault and RaviartGR86 in the case $d=2$ and Section 4.2.4 in Ern and

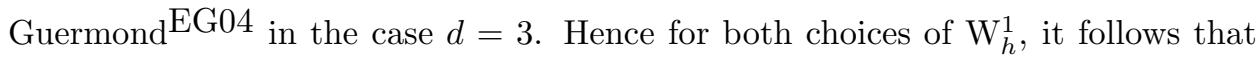
for all $\boldsymbol{v} \in \mathrm{V}$ there exists a sequence $\left\{\boldsymbol{v}_{h}\right\}_{h>0}$, with $\boldsymbol{v}_{h} \in \mathrm{V}_{h}^{1}$, such that

$$
\lim _{h \rightarrow 0_{+}}\left\|\boldsymbol{v}-\boldsymbol{v}_{h}\right\|_{H^{1}(\mathcal{D})}=0 .
$$

We recall the well-known local inverse inequality for $\mathrm{Q}_{h}^{1}$

$$
\begin{gathered}
\|q\|_{L^{\infty}\left(K_{k}\right)} \leq C\left|K_{k}\right|^{-1} \int_{K_{k}}|q| \quad \forall q \in \mathrm{Q}_{h}^{1}, \quad k=1, \ldots, N_{K} \\
\Rightarrow \quad\|\chi\|_{L^{\infty}\left(K_{k}\right)} \leq C\left|K_{k}\right|^{-1} \int_{K_{k}}\|\chi\| \quad \forall \chi \in \mathrm{S}_{h}^{1}, \quad k=1, \ldots, N_{K} .
\end{gathered}
$$

We recall a similar well-known local inverse inequality for $\mathrm{V}_{h}^{1}$

$$
\|\boldsymbol{\nabla} \boldsymbol{v}\|_{L^{2}\left(K_{k}\right)} \leq C h_{k}^{-1}\|\boldsymbol{v}\|_{L^{2}\left(K_{k}\right)} \quad \forall \boldsymbol{v} \in \mathrm{V}_{h}^{1}, \quad k=1, \ldots, N_{K} .
$$

We introduce the interpolation operator $\pi_{h}: C(\overline{\mathcal{D}}) \rightarrow \mathrm{Q}_{h}^{1}$, and extended naturally to $\pi_{h}:[C(\overline{\mathcal{D}})]_{S}^{d \times d} \rightarrow \mathrm{S}_{h}^{1}$, such that for all $\eta \in C(\overline{\mathcal{D}})$ and $\phi \in[C(\overline{\mathcal{D}})]_{S}^{d \times d}$

$$
\pi_{h} \eta\left(P_{p}\right)=\eta\left(P_{p}\right) \quad \text { and } \quad \pi_{h} \phi\left(P_{p}\right)=\phi\left(P_{p}\right) \quad p=1, \ldots, N_{P},
$$

where $\left\{P_{p}\right\}_{p=1}^{N_{P}}$ are the vertices of $\mathcal{T}_{h}$. As $\phi \in \mathrm{S}_{h}^{1}$ does not imply that $G_{\delta}^{(L)^{\prime}}(\phi) \in \mathrm{S}_{h}^{1}$, we have to test the finite element approximation of the $\left(\mathrm{P}_{\alpha, \delta}^{(L)}\right)$ version of (4.1c) 
with $\boldsymbol{I}-\pi_{h}\left[G_{\delta}^{L^{\prime}}\left(\boldsymbol{\sigma}_{\alpha, \delta, h}^{(L,) n}\right)\right] \in \mathrm{S}_{h}^{1}$, where $\boldsymbol{\sigma}_{\alpha, \delta, h}^{(L,) n} \in \mathrm{S}_{h}^{1}$ is our finite element approximation to $\boldsymbol{\sigma}_{\alpha, \delta}^{(L)}$ at time level $t_{n}$. This approximation of the $\left(\mathrm{P}_{\alpha, \delta}^{(L)}\right)$ version of (4.1C) has to be constructed to mimic the results (4.5) -4.8), when tested with $\boldsymbol{I}-\pi_{h}\left[G_{\delta}^{(L)^{\prime}}\left(\boldsymbol{\sigma}_{\alpha, \delta, h}^{(L,) n}\right)\right] \in \mathrm{S}_{h}^{1}$.

In order to mimic (4.5) we shall assume from now on that the family of meshes, $\left\{\mathcal{T}_{h}\right\}_{h>0}$, for the polytope $\mathcal{D}$ consists of non-obtuse simplices only, i.e. all dihedral angles of any simplex in $\mathcal{T}_{h}$ are less than or equal to $\frac{\pi}{2}$. Of course, the construction of such a non-obtuse mesh in the case $d=3$ is not straightforward for a general polytope $\mathcal{D}$. We then have the following result.

Lemma 5.1. Let $g \in C^{0,1}(\mathbb{R})$ be monotonically increasing with Lipschitz constant $g_{\text {Lip. }}$. As $\mathcal{T}_{h}$ consists of only non-obtuse simplices, then we have for all $q \in \mathrm{Q}_{h}^{1}$, $\phi \in \mathrm{S}_{h}^{1}$ that

$$
\begin{array}{r}
g_{\text {Lip }} \boldsymbol{\nabla} \pi_{h}[g(q)] \cdot \boldsymbol{\nabla} q \geq\left\|\boldsymbol{\nabla} \pi_{h}[g(q)]\right\|^{2} \quad \text { and } \quad g_{\text {Lip }} \boldsymbol{\nabla} \pi_{h}[g(\boldsymbol{\phi})]:: \boldsymbol{\nabla} \boldsymbol{\phi} \geq\left\|\boldsymbol{\nabla} \pi_{h}[g(\boldsymbol{\phi})]\right\|^{2} \\
\text { on } K_{k}, \quad k=1, \ldots, N_{K} . \quad \text { (5.7) }
\end{array}
$$

Proof. Let $K_{k}$ have vertices $\left\{P_{j}^{k}\right\}_{j=0}^{d}$, and let $\eta_{j}^{k}(\boldsymbol{x})$ be the basis functions on $K_{k}$ associated with $\mathrm{Q}_{\mathrm{h}}^{1}$ and $\mathrm{S}_{h}^{1}$, i.e. $\left.\eta_{j}^{k}\right|_{K_{k}} \in \mathbb{P}_{1}$ and $\eta_{j}^{k}\left(P_{i}^{k}\right)=\delta_{i j}, i, j=0, \ldots, d$. As $K_{k}$ is non-obtuse it follows that

$$
\boldsymbol{\nabla} \eta_{i}^{k} \cdot \nabla \eta_{j}^{k} \leq 0 \quad \text { on } K_{k}, \quad i, j=0, \ldots, d, \quad \text { with } i \neq j .
$$

We note that

$$
\begin{aligned}
\sum_{j=0}^{d} \eta_{j}^{k} \equiv 1 \quad \text { on } \quad K_{k} & \Rightarrow \\
\left\|\nabla \eta_{i}^{k}\right\|^{2} & =-\sum_{j=0, j \neq i}^{d} \nabla \eta_{i}^{k} \cdot \nabla \eta_{j}^{k} \quad \text { on } \quad K_{k}, \quad i=0, \ldots, d .
\end{aligned}
$$

Hence for $a_{i}, b_{i} \in \mathbb{R}, i=0, \ldots d$, we have that

$$
\begin{aligned}
\boldsymbol{\nabla}\left(\sum_{i=0}^{d} a_{i} \eta_{i}^{k}\right) \cdot \boldsymbol{\nabla}\left(\sum_{j=0}^{d} b_{j} \eta_{j}^{k}\right) & =\sum_{i=0}^{d}\left[a_{i} b_{i}\left\|\nabla \eta_{i}^{k}\right\|^{2}+\sum_{j=0, j \neq i}^{d} a_{i} b_{j} \nabla \eta_{i}^{k} \cdot \nabla \eta_{j}^{k}\right] \\
& =-\sum_{i=0}^{d} \sum_{j=0, j \neq i}^{d} a_{i}\left(b_{i}-b_{j}\right) \nabla \eta_{i}^{k} \cdot \nabla \eta_{j}^{k} \\
& =-\sum_{i=0}^{d} \sum_{j>i}^{d}\left(a_{i}-a_{j}\right)\left(b_{i}-b_{j}\right) \nabla \eta_{i}^{k} \cdot \nabla \eta_{j}^{k} .
\end{aligned}
$$

Similarly for $\boldsymbol{a}_{i}, \boldsymbol{b}_{i} \in \mathbb{R}_{S}^{d \times d}, i=0, \ldots, d$, we have that

$$
\boldsymbol{\nabla}\left(\sum_{i=0}^{d} \boldsymbol{a}_{i} \eta_{i}^{k}\right):: \boldsymbol{\nabla}\left(\sum_{j=0}^{d} \boldsymbol{b}_{j} \eta_{j}^{k}\right)=-\sum_{i=0}^{d} \sum_{j>i}^{d}\left[\left(\boldsymbol{a}_{i}-\boldsymbol{a}_{j}\right):\left(\boldsymbol{b}_{i}-\boldsymbol{b}_{j}\right)\right] \boldsymbol{\nabla} \eta_{i}^{k} \cdot \boldsymbol{\nabla} \eta_{j}^{k}
$$


The desired result (5.7) then follows immediately from (5.10), (5.11), (5.8) and our assumptions on $g$.

In order to mimic (4.6) and (4.8), we need to use numerical integration (vertex sampling). We note the following results. As the basis functions associated with $\mathrm{Q}_{h}^{1}$ and $\mathrm{S}_{h}^{1}$ are nonnegative and sum to unity everywhere, we have for $k=1, \ldots, N_{K}$ that

$$
\left\|\left[\pi_{h} \boldsymbol{\phi}\right](\boldsymbol{x})\right\|^{2} \leq\left(\pi_{h}\left[\|\boldsymbol{\phi}\|^{2}\right]\right)(\boldsymbol{x}) \quad \forall \boldsymbol{x} \in K_{k}, \quad \forall \boldsymbol{\phi} \in\left[C\left(\overline{K_{k}}\right)\right]^{d \times d} .
$$

In addition, we have for $k=1, \ldots, N_{K}$ that

$$
\int_{K_{k}}\|\chi\|^{2} \leq \int_{K_{k}} \pi_{h}\left[\|\chi\|^{2}\right] \leq C \int_{K_{k}}\|\chi\|^{2} \quad \forall \chi \in \mathrm{S}_{h}^{1} .
$$

The first inequality in (5.13) follows immediately from (5.12), and the second from applying (5.4) and a Cauchy-Schwarz inequality.

In order to mimic (4.7), we have to carefully construct our finite element approximation of the convective term in the $\left(\mathrm{P}_{\alpha, \delta}^{(L)}\right)$ version of (4.1c) Our construction is a non-trivial extension of an approach that has been used in the finite element approximation of fourth-order degenerate nonlinear parabolic equations, such as the thin film equation; see e.g. Grün and Rump GR00 and Barrett and Nürnberg! BN04 Let $\left\{\boldsymbol{e}_{i}\right\}_{i=1}^{d}$ be the orthonormal vectors in $\mathbb{R}^{d}$, such that the $j^{\text {th }}$ component of $\boldsymbol{e}_{i}$ is $\delta_{i j}, i, j=1, \ldots, d$. Let $\widehat{K}$ be the standard open reference simplex in $\mathbb{R}^{d}$ with vertices $\left\{\widehat{P}_{i}\right\}_{i=0}^{d}$, where $\widehat{P}_{0}$ is the origin and $\widehat{P}_{i}=\boldsymbol{e}_{i}, i=1, \ldots, d$. Given a simplex $K_{k} \in \mathcal{T}_{h}$ with vertices $\left\{P_{i}^{k}\right\}_{i=0}^{d}$, then there exists a non-singular matrix $B_{k}$ such that the linear mapping

$$
\mathcal{B}_{k}: \widehat{\boldsymbol{x}} \in \mathbb{R}^{d} \mapsto P_{0}^{k}+B_{k} \widehat{\boldsymbol{x}} \in \mathbb{R}^{d}
$$

maps vertex $\widehat{P}_{i}$ to vertex $P_{i}^{k}, i=0, \ldots, d$. Hence $\mathcal{B}_{k}$ maps $\widehat{K}$ to $K_{k}$. For all $\eta \in \mathrm{Q}_{h}^{1}$ and $K_{k} \in \mathcal{T}_{h}$, we define

$$
\widehat{\eta}(\widehat{\boldsymbol{x}}):=\eta\left(\mathcal{B}_{k}(\widehat{\boldsymbol{x}})\right) \quad \forall \widehat{\boldsymbol{x}} \in \widehat{K} \quad \Rightarrow \quad \nabla \eta\left(\mathcal{B}_{k}(\widehat{\boldsymbol{x}})\right)=\left(B_{k}^{T}\right)^{-1} \widehat{\boldsymbol{\nabla}} \widehat{\eta}(\widehat{\boldsymbol{x}}) \quad \forall \widehat{\boldsymbol{x}} \in \widehat{K},
$$

where for all $\widehat{\boldsymbol{x}} \in \widehat{K}$

$$
[\widehat{\nabla} \widehat{\eta}(\widehat{\boldsymbol{x}})]_{j}=\frac{\partial}{\partial \widehat{x}_{j}} \widehat{\eta}(\widehat{\boldsymbol{x}})=\widehat{\eta}\left(\widehat{P}_{j}\right)-\widehat{\eta}\left(\widehat{P}_{0}\right)=\eta\left(P_{j}^{k}\right)-\eta\left(P_{0}^{k}\right) \quad j=1, \ldots, d .
$$

Such notation is easily extended to $\phi \in \mathrm{S}_{h}^{1}$.

Given $\phi \in \mathrm{S}_{h}^{1}$ and $K_{k} \in \mathcal{T}_{h}$, then first, for $j=1, \ldots, d$, we find $\widehat{\Lambda}_{\delta, j}^{(L)}(\widehat{\phi}) \in \mathbb{R}_{S}^{d \times d}$, which depends continuously on $\phi$, such that

$$
\widehat{\Lambda}_{\delta, j}^{(L)}(\widehat{\phi}): \frac{\partial}{\partial \widehat{x}_{j}} \widehat{\pi}_{h}\left[G_{\delta}^{(L)^{\prime}}(\widehat{\phi})\right]=\frac{\partial}{\partial \widehat{x}_{j}} \widehat{\pi}_{h}\left[\operatorname{tr}\left(H_{\delta}^{(L)}\left(G_{\delta}^{(L)^{\prime}}(\widehat{\phi})\right)\right)\right] \quad \text { on } \widehat{K},
$$

where $\left(\widehat{\pi}_{h} \widehat{\eta}\right)(\widehat{\boldsymbol{x}}) \equiv\left(\pi_{h} \eta\right)\left(\mathcal{B}_{k} \widehat{\boldsymbol{x}}\right)$ for all $\widehat{\boldsymbol{x}} \in \widehat{K}$ and $\eta \in C\left(\overline{K_{k}}\right)$. This leads to a unique choice of $\widehat{\Lambda}_{\delta, j}^{(L)}(\widehat{\phi})$. For the construction of $\widehat{\Lambda}_{\delta, j}^{(L)}(\widehat{\phi})$ in the simpler scalar case $(d=1)$, 
see p329 in Barrett and Nürnberg. BN04 To construct $\widehat{\Lambda}_{\delta, j}^{(L)}(\widehat{\phi})$ satisfying (15.17), we note the following. We have from (2.5), (2.6) and (2.15) that

$$
\begin{aligned}
\beta_{\delta}^{(L)}\left(\phi\left(P_{j}^{k}\right)\right):\left(G_{\delta}^{(L)^{\prime}}(\right. & \left.\left.\phi\left(P_{j}^{k}\right)\right)-G_{\delta}^{(L)^{\prime}}\left(\phi\left(P_{0}^{k}\right)\right)\right) \\
& \leq \operatorname{tr}\left(H _ { \delta } ^ { ( L ) } \left(G_{\delta}^{(L)^{\prime}}\left(\phi\left(P_{j}^{k}\right)\right)-H_{\delta}^{(L)}\left(G_{\delta}^{(L)^{\prime}}\left(\phi\left(P_{0}^{k}\right)\right)\right)\right.\right. \\
& \leq \beta_{\delta}^{(L)}\left(\phi\left(P_{0}^{k}\right)\right):\left(G_{\delta}^{(L)^{\prime}}\left(\phi\left(P_{j}^{k}\right)\right)-G_{\delta}^{(L)^{\prime}}\left(\phi\left(P_{0}^{k}\right)\right)\right) .
\end{aligned}
$$

Next we note from (2.5), (2.6), (2.7f) and (1.2b) that

$$
\begin{aligned}
-\left(\beta_{\delta}^{L}\left(\phi\left(P_{j}^{k}\right)\right)-\beta_{\delta}^{L}\left(\phi\left(P_{0}^{k}\right)\right)\right):\left(G_{\delta}^{L^{\prime}}\left(\phi\left(P_{j}^{k}\right)\right)-G_{\delta}^{L^{\prime}}\left(\phi\left(P_{0}^{k}\right)\right)\right) & \\
& \geq L^{-2}\left\|\beta_{\delta}^{L}\left(\phi\left(P_{j}^{k}\right)\right)-\beta_{\delta}^{L}\left(\phi\left(P_{0}^{k}\right)\right)\right\|^{2} ;
\end{aligned}
$$

and so the left-hand side is zero if and only if $\beta_{\delta}^{L}\left(\phi\left(P_{j}^{k}\right)\right)=\beta_{\delta}^{L}\left(\phi\left(P_{0}^{k}\right)\right)$. Similarly, we see from (2.5), (2.6) and the proof of (2.7f); that is, (2.13); that

$$
-\left(\beta_{\delta}\left(\phi\left(P_{j}^{k}\right)\right)-\beta_{\delta}\left(\phi\left(P_{0}^{k}\right)\right)\right):\left(G_{\delta}^{\prime}\left(\phi\left(P_{j}^{k}\right)\right)-G_{\delta}^{\prime}\left(\phi\left(P_{0}^{k}\right)\right)\right) \geq 0
$$

with equality if and only if $\beta_{\delta}\left(\phi\left(P_{j}^{k}\right)\right)=\beta_{\delta}\left(\phi\left(P_{0}^{k}\right)\right)$. Hence, on noting (5.16), (5.18), (5.19), (5.20) and (1.2b), we have that

$$
\begin{aligned}
\widehat{\Lambda}_{\delta, j}^{(L)}(\widehat{\phi}): & \left(1-\lambda_{\delta, j}^{(L)}\right) \beta_{\delta}^{(L)}\left(\phi\left(P_{j}^{k}\right)\right)+\lambda_{\delta, j}^{(L)} \beta_{\delta}^{(L)}\left(\phi\left(P_{0}^{k}\right)\right) \\
& \text { if } \quad\left(\beta_{\delta}^{(L)}\left(\phi\left(P_{j}^{k}\right)\right)-\beta_{\delta}^{(L)}\left(\phi\left(P_{0}^{k}\right)\right)\right):\left(G_{\delta}^{(L)^{\prime}}\left(\phi\left(P_{j}^{k}\right)\right)-G_{\delta}^{(L)^{\prime}}\left(\phi\left(P_{0}^{k}\right)\right)\right) \neq 0, \\
\widehat{\Lambda}_{\delta, j}^{(L)}(\widehat{\phi}): & \beta_{\delta}^{(L)}\left(\phi\left(P_{j}^{k}\right)\right)=\beta_{\delta}^{(L)}\left(\phi\left(P_{0}^{k}\right)\right) \\
& \text { if } \quad\left(\beta_{\delta}^{(L)}\left(\phi\left(P_{j}^{k}\right)\right)-\beta_{\delta}^{(L)}\left(\phi\left(P_{0}^{k}\right)\right)\right):\left(G_{\delta}^{(L)^{\prime}}\left(\phi\left(P_{j}^{k}\right)\right)-G_{\delta}^{(L)^{\prime}}\left(\phi\left(P_{0}^{k}\right)\right)\right)=0
\end{aligned}
$$

satisfies (5.17) for $j=1, \ldots, d$; where $\lambda_{\delta, j}^{(L)} \in[0,1]$ is defined as

$$
\begin{aligned}
\lambda_{\delta, j}^{(L)}:= & {\left[\operatorname { t r } \left(H _ { \delta } ^ { ( L ) } \left(G_{\delta}^{(L)^{\prime}}\left(\phi\left(P_{j}^{k}\right)\right)-H_{\delta}^{(L)}\left(G_{\delta}^{(L)^{\prime}}\left(\phi\left(P_{0}^{k}\right)\right)\right)\right.\right.\right.} \\
& \frac{\left.-\beta_{\delta}^{(L)}\left(\phi\left(P_{j}^{k}\right)\right):\left(G_{\delta}^{(L))^{\prime}}\left(\phi\left(P_{j}^{k}\right)\right)-G_{\delta}^{(L)^{\prime}}\left(\phi\left(P_{0}^{k}\right)\right)\right)\right]}{\left(\beta_{\delta}^{(L)}\left(\phi\left(P_{0}^{k}\right)\right)-\beta_{\delta}^{(L)}\left(\phi\left(P_{j}^{k}\right)\right)\right):\left(G_{\delta}^{(L)^{\prime}}\left(\phi\left(P_{j}^{k}\right)\right)-G_{\delta}^{(L)^{\prime}}\left(\phi\left(P_{0}^{k}\right)\right)\right)} .
\end{aligned}
$$

Furthermore, $\widehat{\Lambda}_{\delta, j}^{(L)}(\widehat{\phi}) \in \mathbb{R}_{S}^{d \times d}, j=1, \ldots, d$, depends continuously on $\left.\phi\right|_{K_{k}}$.

Therefore given $\phi \in \mathrm{S}_{h}^{1}$, we introduce, for $m, p=1, \ldots, d$,

$$
\begin{aligned}
\Lambda_{\delta, m, p}^{(L)}(\phi)=\sum_{j=1}^{d}\left[\left(B_{k}^{T}\right)^{-1}\right]_{m j} \widehat{\Lambda}_{\delta, j}^{(L)}(\widehat{\phi})\left[B_{k}^{T}\right]_{j p} \in \mathbb{R}_{S}^{d \times d} \quad \text { on } K_{k}, & \\
& k=1, \ldots, N_{K} .
\end{aligned}
$$

It follows from (5.22), (5.17) and (5.15) that

$$
\Lambda_{\delta, m, p}^{(L)}(\phi) \approx \beta_{\delta}^{(L)}(\phi) \delta_{m p} \quad m, p=1, \ldots, d ;
$$


and for $m=1, \ldots, d$

$$
\begin{array}{r}
\sum_{p=1}^{d} \Lambda_{\delta, m, p}^{(L)}(\phi): \frac{\partial}{\partial x_{p}} \pi_{h}\left[G_{\delta}^{(L)^{\prime}}(\phi)\right]=\frac{\partial}{\partial x_{m}} \pi_{h}\left[\operatorname{tr}\left(H_{\delta}^{(L)}\left(G_{\delta}^{(L)^{\prime}}(\phi)\right)\right)\right] \quad \text { on } K_{k}, \\
k=1, \ldots, N_{K} .
\end{array}
$$

For a more precise version of (5.23), see Lemma 5.3 below. Finally, as the partitioning $\mathcal{T}_{h}$ consists of regular simplices, we have that

$$
\left\|\left(B_{k}^{T}\right)^{-1}\right\|\left\|B_{k}^{T}\right\| \leq C, \quad k=1, \ldots, N_{K} .
$$

Hence, it follows from (5.22), (5.25), (5.21a,b) and (2.4) that

$$
\left\|\Lambda_{\delta, m, p}^{L}(\phi)\right\|_{L^{\infty}(\mathcal{D})} \leq C L \quad \forall \phi \in \mathrm{S}_{h}^{1} .
$$

\subsection{A free energy preserving approximation, $\left(P_{\alpha, \delta, h}^{(L,) \Delta t}\right)$, of $\left(P_{\alpha, \delta}^{(L)}\right)$}

In addition to the assumptions on the finite element discretization stated in subsection [5.1, and our definition of $\Delta t$ in subsection 3.1 we shall assume that there exists a $C \in \mathbb{R}_{>0}$ such that

$$
\Delta t_{n} \leq C \Delta t_{n-1}, \quad n=2, \ldots, N, \quad \text { as } \quad \Delta t \rightarrow 0_{+} .
$$

With $\Delta t_{1}$ and $C$ as above, let $\Delta t_{0} \in \mathbb{R}_{>0}$ be such that $\Delta t_{1} \leq C \Delta t_{0}$. Given initial data satisfying (2.19), we choose $\boldsymbol{u}_{h}^{0} \in \mathrm{V}_{h}^{1}$ and $\boldsymbol{\sigma}_{h}^{0} \in \mathrm{S}_{h}^{1}$ throughout the rest of this paper such that

$$
\begin{aligned}
\int_{\mathcal{D}}\left[\boldsymbol{u}_{h}^{0} \cdot \boldsymbol{v}+\Delta t_{0} \boldsymbol{\nabla} \boldsymbol{u}_{h}^{0}: \boldsymbol{\nabla} \boldsymbol{v}\right] & =\int_{\mathcal{D}} \boldsymbol{u}^{0} \cdot \boldsymbol{v} & & \forall \boldsymbol{v} \in \mathrm{V}_{h}^{1}, \\
\int_{\mathcal{D}}\left[\pi_{h}\left[\boldsymbol{\sigma}_{h}^{0}: \chi\right]+\Delta t_{0} \boldsymbol{\nabla} \boldsymbol{\sigma}_{h}^{0}:: \nabla \boldsymbol{\nabla}\right] & =\int_{\mathcal{D}} \boldsymbol{\sigma}^{0}: \chi & & \forall \chi \in \mathrm{S}_{h}^{1} .
\end{aligned}
$$

It follows from (5.28a,b), (5.13) and (2.19) that

$$
\int_{\mathcal{D}}\left[\left\|\boldsymbol{u}_{h}^{0}\right\|^{2}+\left\|\boldsymbol{\sigma}_{h}^{0}\right\|^{2}+\Delta t_{0}\left[\left\|\nabla \boldsymbol{u}_{h}^{0}\right\|^{2}+\left\|\nabla \boldsymbol{\sigma}_{h}^{0}\right\|^{2}\right]\right] \leq C .
$$

In addition, we note the following result.

Lemma 5.2. For $p=1, \ldots, N_{P}$ we have that

$$
\sigma_{\min }^{0}\|\boldsymbol{\xi}\|^{2} \leq \boldsymbol{\xi}^{T} \boldsymbol{\sigma}_{h}^{0}\left(P_{p}\right) \boldsymbol{\xi} \leq \sigma_{\max }^{0}\|\boldsymbol{\xi}\|^{2} \quad \forall \boldsymbol{\xi} \in \mathbb{R}^{d} .
$$

Proof. It follows from (5.28b) that

$$
\begin{array}{r}
\int_{\mathcal{D}}\left[\pi_{h}\left[\left(\boldsymbol{\sigma}_{h}^{0}-\boldsymbol{\sigma}_{\min }^{0} \boldsymbol{I}\right): \boldsymbol{\chi}\right]+\Delta t_{0} \boldsymbol{\nabla}\left(\boldsymbol{\sigma}_{h}^{0}-\boldsymbol{\sigma}_{\min }^{0} \boldsymbol{I}\right):: \boldsymbol{\nabla} \boldsymbol{\chi}\right]=\int_{\mathcal{D}}\left(\boldsymbol{\sigma}^{0}-\boldsymbol{\sigma}_{\min }^{0} \boldsymbol{I}\right): \boldsymbol{\chi} \\
\forall \boldsymbol{\chi} \in \mathrm{S}_{h}^{1} .
\end{array}
$$

Choosing $\chi=\boldsymbol{\xi} \boldsymbol{\xi}^{T} \eta$, with $\eta \in \mathrm{Q}_{h}^{1}$ yields that $z_{h}:=\boldsymbol{\xi}^{T}\left(\boldsymbol{\sigma}_{h}^{0}-\boldsymbol{\sigma}_{\min }^{0} \boldsymbol{I}\right) \boldsymbol{\xi} \in \mathrm{Q}_{h}^{1}$ satisfies

$$
\int_{\mathcal{D}}\left[\pi_{h}\left[z_{h} \eta\right]+\Delta t_{0} \nabla z_{h} \cdot \nabla \eta\right]=\int_{\mathcal{D}} z \eta \quad \forall \eta \in \mathrm{Q}_{1}^{h},
$$


where $z:=\boldsymbol{\xi}^{T}\left(\boldsymbol{\sigma}^{0}-\boldsymbol{\sigma}_{\min }^{0} \boldsymbol{I}\right) \boldsymbol{\xi} \in L^{\infty}(\mathcal{D})$ and is non-negative on recalling (2.19).

Choosing $\eta=\pi_{h}\left[z_{h}\right]_{-} \in \mathrm{Q}_{h}^{1}$, it follows, on noting the $\mathrm{Q}_{h}^{1}$ version of (5.13) and (5.7) with $g(\cdot)=[\cdot]_{-}$, that

$$
\begin{aligned}
\left.\int_{\mathcal{D}}\left[\pi_{h}\left[z_{h}\right]_{-}\right]^{2}+\Delta t_{0}\left\|\nabla \pi_{h}\left[z_{h}\right]_{-}\right\|^{2}\right] & \leq \int_{\mathcal{D}}\left[\pi_{h}\left[\left[z_{h}\right]_{-}^{2}\right]+\Delta t_{0} \nabla z_{h} \cdot \nabla \pi_{h}\left[z_{h}\right]_{-}\right] \\
& =\int_{\mathcal{D}} z \pi_{h}\left[z_{h}\right]_{-} \leq 0 .
\end{aligned}
$$

Hence $\pi_{h}\left[z_{h}\right]_{-} \equiv 0$ and so the first inequality in (5.30) holds. Repeating the above with $\boldsymbol{\sigma}_{\min }^{0}$ and $[\cdot]_{-}$replaced by $\boldsymbol{\sigma}_{\max }^{0}$ and $[\cdot]_{+}$, respectively, yields the second inequality in (5.30).

Our approximation $\left(\mathrm{P}_{\alpha, \delta, h}^{(L,) \Delta t}\right)$ of $\left(\mathrm{P}_{\alpha, \delta}^{(L)}\right)$ is then:

$\left(\mathbf{P}_{\alpha, \delta, h}^{(L,) \Delta t}\right)$ Setting $\left(\boldsymbol{u}_{\alpha, \delta, h}^{(L), 0}, \boldsymbol{\sigma}_{\alpha, \delta, h}^{(L), 0}\right)=\left(\boldsymbol{u}_{h}^{0}, \boldsymbol{\sigma}_{h}^{0}\right) \in \mathrm{V}_{h}^{1} \times \mathrm{S}_{h}^{1}$, then for $n=1, \ldots, N_{T}$ find $\left(\boldsymbol{u}_{\alpha, \delta, h}^{(L,) n}, \boldsymbol{\sigma}_{\alpha, \delta, h}^{(L,) n}\right) \in \mathrm{V}_{h}^{1} \times \mathrm{S}_{h}^{1}$ such that for any test functions $(\boldsymbol{v}, \boldsymbol{\phi}) \in \mathrm{V}_{h}^{1} \times \mathrm{S}_{h}^{1}$

$$
\begin{aligned}
& \int_{\mathcal{D}}\left[\operatorname{Re}\left(\frac{\boldsymbol{u}_{\alpha, \delta, h}^{(L,) n}-\boldsymbol{u}_{\alpha, \delta, h}^{(L,) n-1}}{\Delta t_{n}}\right) \cdot \boldsymbol{v}\right. \\
& \quad+\frac{\operatorname{Re}}{2}\left[\left(\left(\boldsymbol{u}_{\alpha, \delta, h}^{(L,) n-1} \cdot \nabla\right) \boldsymbol{u}_{\alpha, \delta, h}^{(L,) n}\right) \cdot \boldsymbol{v}-\boldsymbol{u}_{\alpha, \delta, h}^{(L,) n} \cdot\left(\left(\boldsymbol{u}_{\alpha, \delta, h}^{(L,) n-1} \cdot \nabla\right) \boldsymbol{v}\right)\right] \\
& \left.\quad+(1-\varepsilon) \boldsymbol{\nabla} \boldsymbol{u}_{\alpha, \delta, h}^{(L,) n}: \nabla \boldsymbol{v}+\frac{\varepsilon}{\mathrm{Wi}} \pi_{h}\left[\beta_{\delta}^{(L)}\left(\boldsymbol{\sigma}_{\alpha, \delta, h}^{(L,) n}\right)\right]: \nabla \boldsymbol{v}\right]=\left\langle\boldsymbol{f}^{n}, \boldsymbol{v}\right\rangle_{H_{0}^{1}(\mathcal{D})}, \\
& \quad+\int_{D}\left[\alpha \boldsymbol{\nabla} \boldsymbol{\sigma}_{\alpha, \delta, h}^{(L,) n}:: \nabla \boldsymbol{\phi}-2 \boldsymbol{\nabla} \boldsymbol{u}_{\alpha, \delta, h}^{(L,) n}: \pi_{h}\left[\boldsymbol{\phi} \beta_{\delta}^{(L)}\left(\boldsymbol{\sigma}_{\alpha, \delta, h}^{(L,) n}\right)\right]\right. \\
& \quad-\int_{\mathcal{D}}\left[\left(\sum_{m=1}^{d} \sum_{p=1}^{d L,) n}\left[\boldsymbol{\sigma}_{\alpha, \delta, h}^{(L,) n-1}{ }^{(L,) n-1}\right]_{m} \Lambda_{\delta, m, p}^{(L)}\left(\boldsymbol{\sigma}_{\alpha, \delta, h}^{(L,) n}\right): \frac{\partial \boldsymbol{\phi}}{\partial \boldsymbol{x}_{p}}=0 .\right.\right.
\end{aligned}
$$

In deriving $\left(\mathrm{P}_{\alpha, \delta, h}^{(L,) \Delta t}\right)$, we have noted (3.13), (1.4a) and (5.22).

Before proving existence of a solution to $\left(\mathrm{P}_{\alpha, \delta, h}^{(L,) \Delta t}\right)$, we first derive a discrete analogue of the energy estimate (4.3) for $\left(\mathrm{P}_{\alpha, \delta}^{(L)}\right)$.

\subsection{Energy estimate}

On setting

$$
\begin{array}{r}
F_{\delta, h}^{(L)}(\boldsymbol{v}, \phi):=\frac{\operatorname{Re}}{2} \int_{\mathcal{D}}\|\boldsymbol{v}\|^{2}+\frac{\varepsilon}{2 \mathrm{Wi}} \int_{\mathcal{D}} \pi_{h}\left[\operatorname{tr}\left(\boldsymbol{\phi}-G_{\delta}^{(L)}(\boldsymbol{\phi})-\boldsymbol{I}\right)\right] \\
\forall(\boldsymbol{v}, \boldsymbol{\phi}) \in \mathrm{V}_{h}^{1} \times \mathrm{S}_{h}^{1},
\end{array}
$$

we have the following discrete analogue of Proposition 4.1 
Proposition 5.1. For $n=1, \ldots, N_{T}$, a solution $\left(\boldsymbol{u}_{\alpha, \delta, h}^{(L,) n}, \boldsymbol{\sigma}_{\alpha, \delta, h}^{(L,) n}\right) \in \mathrm{V}_{h}^{1} \times \mathrm{S}_{h}^{1}$ to (5.34a, b), if it exists, satisfies

$$
\begin{gathered}
\frac{F_{\delta, h}^{(L)}\left(\boldsymbol{u}_{\alpha, \delta, h}^{(L,) n}, \boldsymbol{\sigma}_{\alpha, \delta, h}^{(L,) n}\right)-F_{\delta, h}^{(L)}\left(\boldsymbol{u}_{\alpha, \delta, h}^{(L,) n-1}, \boldsymbol{\sigma}_{\alpha, \delta, h}^{(L,) n-1}\right)}{\Delta t_{n}}+\frac{\operatorname{Re}}{2 \Delta t_{n}} \int_{\mathcal{D}}\left\|\boldsymbol{u}_{\alpha, \delta, h}^{(L,) n}-\boldsymbol{u}_{\alpha, \delta, h}^{(L,) n-1}\right\|^{2} \\
+(1-\varepsilon) \int_{\mathcal{D}}\left\|\boldsymbol{\nabla} \boldsymbol{u}_{\alpha, \delta, h}^{(L,) n}\right\|^{2}+\frac{\varepsilon}{2 \mathrm{Wi}^{2}} \int_{\mathcal{D}} \pi_{h}\left[\operatorname{tr}\left(\beta_{\delta}^{(L)}\left(\boldsymbol{\sigma}_{\alpha, \delta, h}^{(L,) n}\right)+\left[\beta_{\delta}^{(L)}\left(\boldsymbol{\sigma}_{\alpha, \delta, h}^{(L,) n}\right)\right]^{-1}-2 \boldsymbol{I}\right)\right] \\
+\frac{\alpha \varepsilon \delta^{2}}{2 \mathrm{Wi}} \int_{\mathcal{D}}\left\|\boldsymbol{\nabla} \pi_{h}\left[G_{\delta}^{(L)}\left(\boldsymbol{\sigma}_{\alpha, \delta, h}^{(L,) n}\right)\right]\right\|^{2} \\
\leq\left\langle\boldsymbol{f}^{n}, \boldsymbol{u}_{\alpha, \delta, h}^{(L,) n}\right\rangle_{H_{0}^{1}(\mathcal{D})} \\
\leq \frac{1}{2}(1-\varepsilon) \int_{\mathcal{D}}\left\|\boldsymbol{\nabla} \boldsymbol{u}_{\alpha, \delta, h}^{(L,) n}\right\|^{2}+\frac{1+C_{P}}{2(1-\varepsilon)}\left\|\boldsymbol{f}^{n}\right\|_{H^{-1}(\mathcal{D})}^{2} .
\end{gathered}
$$

Proof. The proof is similar to that of Proposition 3.1 we choose as test functions $\boldsymbol{v}=\boldsymbol{u}_{\alpha, \delta, h}^{(L,) n} \in \mathrm{V}_{h}^{1}$ and $\boldsymbol{\phi}=\frac{\varepsilon}{2 \mathrm{Wi}}\left(\boldsymbol{I}-\pi_{h}\left[G_{\delta}^{(L)^{\prime}}\left(\boldsymbol{\sigma}_{\alpha, \delta, h}^{(L,) n}\right)\right]\right) \in \mathrm{S}_{h}^{1}$ in (5.34a, b), and obtain, on noting (3.14), (2.7a), d,e), (5.7) with $g=-G_{\delta}^{(L)^{\prime}}$ having Lipschitz constant $\delta^{-2},(5.24)$ and (5.35) that

$$
\begin{aligned}
\left\langle\boldsymbol{f}^{n}, \boldsymbol{u}_{\alpha, \delta, h}^{(L,) n}\right\rangle_{H_{0}^{1}(\mathcal{D})} & \\
\geq & \frac{F_{\delta, h}^{(L)}\left(\boldsymbol{u}_{\alpha, \delta, h}^{(L,) n}, \boldsymbol{\sigma}_{\alpha, \delta, h}^{(L,) n}\right)-F_{\delta, h}^{(L)}\left(\boldsymbol{u}_{\alpha, \delta, h}^{(L,) n-1}, \boldsymbol{\sigma}_{\alpha, \delta, h}^{(L,) n-1}\right)}{\Delta t_{n}}+(1-\varepsilon) \int_{\mathcal{D}}\left\|\boldsymbol{\nabla} \boldsymbol{u}_{\alpha, \delta, h}^{(L,) n}\right\|^{2} \\
& +\frac{\operatorname{Re}}{2 \Delta t_{n}} \int_{\mathcal{D}}\left\|\boldsymbol{u}_{\alpha, \delta, h}^{(L,) n}-\boldsymbol{u}_{\alpha, \delta, h}^{(L,) n-1}\right\|^{2}+\frac{\alpha \varepsilon \delta^{2}}{2 \mathrm{Wi}} \int_{\mathcal{D}}\left\|\boldsymbol{\nabla} \pi_{h}\left[G_{\delta}^{(L))^{\prime}}\left(\boldsymbol{\sigma}_{\alpha, \delta, h}^{(L,)}\right)\right]\right\|^{2} \\
& +\frac{\varepsilon}{2 \mathrm{Wi}^{2}} \int_{\mathcal{D}} \pi_{h}\left[\operatorname{tr}\left(\beta_{\delta}^{(L)}\left(\boldsymbol{\sigma}_{\alpha, \delta, h}^{(L,) n}\right)+\left[\beta_{\delta}^{(L)}\left(\boldsymbol{\sigma}_{\alpha, \delta, h}^{(L,) n}\right)\right]^{-1}-2 \boldsymbol{I}\right)\right] \\
& +\int_{\mathcal{D}} \boldsymbol{u}_{\alpha, \delta, h}^{(L,) n-1} \cdot \boldsymbol{\nabla} \pi_{h}\left[\operatorname{tr}\left(H_{\delta}^{(L)}\left(G_{\delta}^{(L) \prime}\left(\boldsymbol{\sigma}_{\alpha, \delta, h}^{(L,) n}\right)\right)\right]\right.
\end{aligned}
$$

The first desired inequality in (5.36) follows immediately from (5.37) on noting (5.1d), (3.2a), (1.9) and that $\pi_{h}: C(\overline{\mathcal{D}}) \rightarrow \mathrm{Q}_{h}^{1}$. The second inequality in (5.36) follows immediately from (2.28) with $\nu^{2}=(1-\varepsilon) /\left(1+C_{P}\right)$.

\subsection{Existence of discrete solutions}

Proposition 5.2. Given $\left(\boldsymbol{u}_{\alpha, \delta, h}^{(L,) n-1}, \boldsymbol{\sigma}_{\alpha, \delta, h}^{(L,) n-1}\right) \in \mathrm{V}_{h}^{1} \times \mathrm{S}_{h}^{1}$ and for any time step $\Delta t_{n}>0$, then there exists at least one solution $\left(\boldsymbol{u}_{\alpha, \delta, h}^{(L,) n}, \boldsymbol{\sigma}_{\alpha, \delta, h}^{(L,) n}\right) \in \mathrm{V}_{h}^{1} \times \mathrm{S}_{h}^{1}$ to $5.34 a, b)$.

Proof. The proof is similar to that of Proposition 3.2. We introduce the following inner product on the Hilbert space $\mathrm{V}_{h}^{1} \times \mathrm{S}_{h}^{1}$

$$
((\boldsymbol{w}, \boldsymbol{\psi}),(\boldsymbol{v}, \boldsymbol{\phi}))_{\mathcal{D}}^{h}=\int_{\mathcal{D}}\left[\boldsymbol{w} \cdot \boldsymbol{v}+\pi_{h}[\boldsymbol{\psi}: \boldsymbol{\phi}]\right] \quad \forall(\boldsymbol{w}, \boldsymbol{\psi}),(\boldsymbol{v}, \boldsymbol{\phi}) \in \mathrm{V}_{h}^{1} \times \mathrm{S}_{h}^{1}
$$


Given $\left(\boldsymbol{u}_{\alpha, \delta, h}^{(L,) n-1}, \boldsymbol{\sigma}_{\alpha, \delta, h}^{(L,) n-1}\right) \in \mathrm{V}_{h}^{1} \times \mathrm{S}_{h}^{1}$, let $\mathcal{F}^{h}: \mathrm{V}_{h}^{1} \times \mathrm{S}_{h}^{1} \rightarrow \mathrm{V}_{h}^{1} \times \mathrm{S}_{h}^{1}$ be such that for any $(\boldsymbol{w}, \boldsymbol{\psi}) \in \mathrm{V}_{h}^{1} \times \mathrm{S}_{h}^{1}$

$$
\begin{aligned}
&\left(\mathcal{F}^{h}(\boldsymbol{w}, \boldsymbol{\psi}),(\boldsymbol{v}, \boldsymbol{\phi})\right)_{\mathcal{D}}^{h} \\
&:= \int_{\mathcal{D}}\left[\operatorname{Re}\left(\frac{\boldsymbol{w}-\boldsymbol{u}_{\alpha, \delta, h}^{(L,) n-1}}{\Delta t_{n}}\right) \cdot \boldsymbol{v}+(1-\varepsilon) \boldsymbol{\nabla} \boldsymbol{w}: \boldsymbol{\nabla} \boldsymbol{v}+\frac{\varepsilon}{\mathrm{Wi}} \pi_{h}\left[\beta_{\delta}^{(L)}(\boldsymbol{\psi})\right]: \boldsymbol{\nabla} \boldsymbol{v}\right. \\
&+\frac{\operatorname{Re}}{2}\left[\left(\left(\boldsymbol{u}_{\alpha, \delta, h}^{(L,) n-1} \cdot \nabla\right) \boldsymbol{w}\right) \cdot \boldsymbol{v}-\boldsymbol{w} \cdot\left(\left(\boldsymbol{u}_{\alpha, \delta, h}^{(L,) n-1} \cdot \nabla\right) \boldsymbol{v}\right)\right]+\alpha \boldsymbol{\nabla} \boldsymbol{\psi}:: \boldsymbol{\nabla} \boldsymbol{\phi} \\
&\left.-2 \boldsymbol{\nabla} \boldsymbol{w}: \pi_{h}\left[\boldsymbol{\phi} \beta_{\delta}^{(L)}(\boldsymbol{\psi})\right]+\pi_{h}\left[\left(\frac{\boldsymbol{\psi}-\boldsymbol{\sigma}_{\alpha, \delta, h}^{(L,) n-1}}{\Delta t_{n}}\right): \boldsymbol{\phi}+\frac{1}{\mathrm{Wi}}(\boldsymbol{\psi}-\boldsymbol{I}): \boldsymbol{\phi}\right]\right] \\
&-\int_{\mathcal{D}} \sum_{m=1}^{d} \sum_{p=1}^{d}\left[\boldsymbol{u}_{\alpha, \delta, h}^{(L,) n-1}\right]_{m} \Lambda_{\delta, m, p}^{(L)}(\boldsymbol{\psi}): \frac{\partial \boldsymbol{\phi}}{\partial \boldsymbol{x}_{p}}-\left\langle\boldsymbol{f}^{n}, \boldsymbol{v}\right\rangle_{H_{0}^{1}(\mathcal{D})} \\
& \forall(\boldsymbol{v}, \boldsymbol{\phi}) \in \mathrm{V}_{h}^{1} \times \mathrm{S}_{h}^{1} .
\end{aligned}
$$

A solution $\left(\boldsymbol{u}_{\alpha, \delta, h}^{(L,) n}, \boldsymbol{\sigma}_{\alpha, \delta, h}^{(L,) n}\right)$ to (3.12a $\left.\mathrm{b}\right)$, if it exists, corresponds to a zero of $\mathcal{F}^{h}$. On recalling (5.22) and (5.21a,b), it is easily deduced that the mapping $\mathcal{F}^{h}$ is continuous.

For any $(\boldsymbol{w}, \boldsymbol{\psi}) \in \mathrm{V}_{h}^{1} \times \mathrm{S}_{h}^{1}$, on choosing $(\boldsymbol{v}, \phi)=\left(\boldsymbol{w}, \frac{\varepsilon}{2 \mathrm{Wi}}\left(\boldsymbol{I}-\pi_{h}\left[G_{\delta}^{(L)^{\prime}}(\boldsymbol{\psi})\right]\right)\right)$, we obtain analogously to (5.36) that

$$
\begin{gathered}
\left(\mathcal{F}^{h}(\boldsymbol{w}, \boldsymbol{\psi}),\left(\boldsymbol{w}, \frac{\varepsilon}{2 \mathrm{Wi}}\left(\boldsymbol{I}-\pi_{h}\left[G_{\delta}^{(L)^{\prime}}(\boldsymbol{\psi})\right]\right)\right)\right)_{\mathcal{D}}^{h} \\
\geq \frac{F_{\delta, h}^{(L)}(\boldsymbol{w}, \boldsymbol{\psi})-F_{\delta, h}^{(L)}\left(\boldsymbol{u}_{\alpha, \delta, h}^{(L,) n-1}, \boldsymbol{\sigma}_{\alpha, \delta, h}^{(L,) n-1}\right)}{\Delta t_{n}}+\frac{\operatorname{Re}}{2 \Delta t_{n}} \int_{\mathcal{D}}\left\|\boldsymbol{w}-\boldsymbol{u}_{\alpha, \delta, h}^{(L,) n-1}\right\|^{2} \\
\quad+\frac{1-\varepsilon}{2} \int_{\mathcal{D}}\|\boldsymbol{\nabla} \boldsymbol{w}\|^{2}+\frac{\varepsilon}{2 \mathrm{Wi}^{2}} \int_{\mathcal{D}} \pi_{h}\left[\operatorname{tr}\left(\beta_{\delta}^{(L)}(\boldsymbol{\psi})+\left[\beta_{\delta}^{(L)}(\boldsymbol{\psi})\right]^{-1}-2 \boldsymbol{I}\right)\right] \\
\quad+\frac{\alpha \varepsilon \delta^{2}}{2 \mathrm{Wi}} \int_{\mathcal{D}}\left\|\nabla \pi_{h}\left[G_{\delta}^{(L)}(\boldsymbol{\psi})\right]\right\|^{2}-\frac{1+C_{P}}{2(1-\varepsilon)}\left\|\boldsymbol{f}^{n}\right\|_{H^{-1}(\mathcal{D})}^{2}
\end{gathered}
$$

Let

$$
\|(\boldsymbol{v}, \phi)\|_{D}^{h}:=\left[((\boldsymbol{v}, \phi),(\boldsymbol{v}, \phi))_{D}^{h}\right]^{\frac{1}{2}}=\left(\int_{\mathcal{D}}\left[\|\boldsymbol{v}\|^{2}+\pi_{h}\left[\|\boldsymbol{\phi}\|^{2}\right]\right]\right)^{\frac{1}{2}} .
$$

If for any $\gamma \in \mathbb{R}_{>0}$, the continuous mapping $\mathcal{F}^{h}$ has no zero $\left(\boldsymbol{u}_{\alpha, \delta, h}^{(L,) n}, \boldsymbol{\sigma}_{\alpha, \delta, h}^{(L,) n}\right)$, which lies in the ball

$$
\mathcal{B}_{\gamma}^{h}:=\left\{(\boldsymbol{v}, \phi) \in \mathrm{V}_{h}^{1} \times \mathrm{S}_{h}^{1}:\|(\boldsymbol{v}, \phi)\|_{\mathcal{D}}^{h} \leq \gamma\right\} ;
$$

then for such $\gamma$, we can define the continuous mapping $\mathcal{G}_{\gamma}^{h}: \mathcal{B}_{\gamma}^{h} \rightarrow \mathcal{B}_{\gamma}^{h}$ such that for all $(\boldsymbol{v}, \phi) \in \mathcal{B}_{\gamma}^{h}$

$$
\mathcal{G}_{\gamma}^{h}(\boldsymbol{v}, \phi):=-\gamma \frac{\mathcal{F}^{h}(\boldsymbol{v}, \boldsymbol{\phi})}{\left\|\mathcal{F}^{h}(\boldsymbol{v}, \phi)\right\|_{\mathcal{D}}^{h}}
$$


By the Brouwer fixed point theorem, $\mathcal{G}_{\gamma}^{h}$ has at least one fixed point $\left(\boldsymbol{w}_{\gamma}, \boldsymbol{\psi}_{\gamma}\right)$ in $\mathcal{B}_{\gamma}^{h}$. Hence it satisfies

$$
\left\|\left(\boldsymbol{w}_{\gamma}, \boldsymbol{\psi}_{\gamma}\right)\right\|_{\mathcal{D}}^{h}=\left\|\mathcal{G}_{\gamma}^{h}\left(\boldsymbol{w}_{\gamma}, \boldsymbol{\psi}_{\gamma}\right)\right\|_{\mathcal{D}}^{h}=\gamma .
$$

On noting (5.4), we have that there exists a $\mu_{h} \in \mathbb{R}_{>0}$ such that for all $\phi \in \mathrm{S}_{h}^{1}$,

$$
\left\|\pi_{h}[\|\phi\|]\right\|_{L^{\infty}(\mathcal{D})}^{2} \leq\left\|\pi_{h}\left[\|\phi\|^{2}\right]\right\|_{L^{\infty}(\mathcal{D})} \leq \mu_{h}^{2} \int_{\mathcal{D}} \pi_{h}\left[\|\phi\|^{2}\right] .
$$

It follows from (5.35), (2.8), (5.41) and (5.40) that

$$
\begin{aligned}
F_{\delta, h}^{(L)}\left(\boldsymbol{w}_{\gamma},\right. & \left.\boldsymbol{\psi}_{\gamma}\right) \\
& =\frac{\operatorname{Re}}{2} \int_{\mathcal{D}}\left\|\boldsymbol{w}_{\gamma}\right\|^{2}+\frac{\varepsilon}{2 \mathrm{Wi}} \int_{\mathcal{D}} \pi_{h}\left[\operatorname{tr}\left(\boldsymbol{\psi}_{\gamma}-G_{\delta}^{(L)}\left(\boldsymbol{\psi}_{\gamma}\right)-\boldsymbol{I}\right)\right] \\
& \geq \frac{\operatorname{Re}}{2} \int_{\mathcal{D}}\left\|\boldsymbol{w}_{\gamma}\right\|^{2}+\frac{\varepsilon}{4 \mathrm{Wi}}\left[\int_{\mathcal{D}} \pi_{h}\left[\left\|\boldsymbol{\psi}_{\gamma}\right\|\right]-2 d|\mathcal{D}|\right] \\
& \geq \frac{\operatorname{Re}}{2} \int_{\mathcal{D}}\left\|\boldsymbol{w}_{\gamma}\right\|^{2}+\frac{\varepsilon}{4 \mathrm{Wi} \mu_{h} \gamma}\left\|\pi_{h}\left[\left\|\boldsymbol{\psi}_{\gamma}\right\|\right]\right\|_{L^{\infty}(\mathcal{D})}\left[\int_{\mathcal{D}} \pi_{h}\left[\left\|\boldsymbol{\psi}_{\gamma}\right\|\right]\right]-\frac{\varepsilon d|\mathcal{D}|}{2 \mathrm{Wi}} \\
& \geq \min \left(\frac{\operatorname{Re}}{2}, \frac{\varepsilon}{4 \mathrm{Wi} \mu_{h} \gamma}\right)\left(\int_{\mathcal{D}}\left[\left\|\boldsymbol{w}_{\gamma}\right\|^{2}+\pi_{h}\left[\left\|\boldsymbol{\psi}_{\gamma}\right\|^{2}\right]\right]\right)-\frac{\varepsilon d|\mathcal{D}|}{2 \mathrm{Wi}} \\
& =\min \left(\frac{\operatorname{Re}}{2}, \frac{\varepsilon}{4 \mathrm{Wi} \mu_{h} \gamma}\right) \gamma^{2}-\frac{\varepsilon d|\mathcal{D}|}{2 \mathrm{Wi}}
\end{aligned}
$$

Hence for all $\gamma$ sufficiently large, it follows from (5.39) and (5.42) that

$$
\left(\mathcal{F}^{h}\left(\boldsymbol{w}_{\gamma}, \boldsymbol{\psi}_{\gamma}\right),\left(\boldsymbol{w}_{\gamma}, \frac{\varepsilon}{2 \mathrm{Wi}}\left(\boldsymbol{I}-\pi_{h}\left[G_{\delta}^{(L)^{\prime}}\left(\boldsymbol{\psi}_{\gamma}\right)\right]\right)\right)\right)_{\mathcal{D}}^{h} \geq 0
$$

On the other hand as $\left(\boldsymbol{w}_{\gamma}, \boldsymbol{\psi}_{\gamma}\right)$ is a fixed point of $\mathcal{G}_{\gamma}^{h}$, we have that

$$
\begin{aligned}
& \left(\mathcal{F}^{h}\left(\boldsymbol{w}_{\gamma}, \boldsymbol{\psi}_{\gamma}\right),\left(\boldsymbol{w}_{\gamma}, \frac{\varepsilon}{2 \mathrm{Wi}}\left(\boldsymbol{I}-\pi_{h}\left[G_{\delta}^{(L)^{\prime}}\left(\boldsymbol{\psi}_{\gamma}\right)\right]\right)\right)\right)_{D}^{h} \\
& \quad=-\frac{\left\|\mathcal{F}^{h}\left(\boldsymbol{w}_{\gamma}, \boldsymbol{\psi}_{\gamma}\right)\right\|_{\mathcal{D}}^{h}}{\gamma} \int_{\mathcal{D}}\left[\left\|\boldsymbol{w}_{\gamma}\right\|^{2}+\frac{\varepsilon}{2 \mathrm{Wi}} \pi_{h}\left[\boldsymbol{\psi}_{\gamma}:\left(\boldsymbol{I}-G_{\delta}^{(L)^{\prime}}\left(\boldsymbol{\psi}_{\gamma}\right)\right)\right]\right] .
\end{aligned}
$$

It follows from (2.8), and similarly to (5.42), on noting (5.41) and (5.40) that

$$
\begin{aligned}
& \int_{\mathcal{D}}\left[\left\|\boldsymbol{w}_{\gamma}\right\|^{2}+\frac{\varepsilon}{2 \mathrm{Wi}} \pi_{h}\left[\boldsymbol{\psi}_{\gamma}:\left(\boldsymbol{I}-G_{\delta}^{(L)^{\prime}}\left(\boldsymbol{\psi}_{\gamma}\right)\right)\right]\right] \\
& \geq \int_{\mathcal{D}}\left[\left\|\boldsymbol{w}_{\gamma}\right\|^{2}+\frac{\varepsilon}{4 \mathrm{Wi}}\left[\pi_{h}\left[\left\|\boldsymbol{\psi}_{\gamma}\right\|\right]-2 d\right]\right] \\
& \geq \min \left(1, \frac{\varepsilon}{4 \mathrm{Wi} \mu_{h} \gamma}\right) \gamma^{2}-\frac{\varepsilon d|\mathcal{D}|}{2 \mathrm{Wi}} .
\end{aligned}
$$

Therefore on combining (5.44) and (5.45), we have for all $\gamma$ sufficiently large that

$$
\left(\mathcal{F}^{h}\left(\boldsymbol{w}_{\gamma}, \boldsymbol{\psi}_{\gamma}\right),\left(\boldsymbol{w}_{\gamma}, \frac{\varepsilon}{2 \mathrm{Wi}}\left(\boldsymbol{I}-\pi_{h}\left[G_{\delta}^{(L)^{\prime}}\left(\boldsymbol{\psi}_{\gamma}\right)\right]\right)\right)\right)_{D}^{h}<0,
$$


which obviously contradicts (5.43). Hence the mapping $\mathcal{F}^{h}$ has a zero in $\mathcal{B}_{\gamma}^{h}$ for $\gamma$ sufficiently large.

We now have the analogue of stability Theorem 3.1

Theorem 5.1. For any $\delta \in\left(0, \frac{1}{2}\right], L \geq 2, N_{T} \geq 1$ and any partitioning of $[0, T]$ into $N_{T}$ time steps, there exists a solution $\left\{\left(\boldsymbol{u}_{\alpha, \delta, h}^{(L), n}, \boldsymbol{\sigma}_{\alpha, \delta, h}^{(L), n}\right)\right\}_{n=1}^{N_{T}} \in\left[\mathrm{V}_{h}^{1} \times \mathrm{S}_{h}^{1}\right]^{N_{T}}$ to $\left(P_{\alpha, \delta, h}^{(L,) \Delta t}\right)$.

In addition, it follows for $n=1, \ldots, N_{T}$ that

$$
\begin{gathered}
F_{\delta, h}^{(L)}\left(\boldsymbol{u}_{\alpha, \delta, h}^{(L,) n}, \boldsymbol{\sigma}_{\alpha, \delta, h}^{(L,) n}\right)+\frac{\alpha \varepsilon \delta^{2}}{2 \mathrm{Wi}} \sum_{m=1}^{n} \Delta t_{m} \int_{\mathcal{D}}\left\|\boldsymbol{\nabla} \pi_{h}\left[G_{\delta}^{(L)^{\prime}}\left(\boldsymbol{\sigma}_{\alpha, \delta, h}^{(L,) m}\right)\right]\right\|^{2} \\
+\frac{1}{2} \sum_{m=1}^{n} \int_{\mathcal{D}}\left[\operatorname{Re}\left\|\boldsymbol{u}_{\alpha, \delta, h}^{(L,) m}-\boldsymbol{u}_{\alpha, \delta, h}^{(L,) m-1}\right\|^{2}+(1-\varepsilon) \Delta t_{m}\left\|\boldsymbol{\nabla} \boldsymbol{u}_{\alpha, \delta, h}^{(L,) m}\right\|^{2}\right] \\
+\frac{\varepsilon}{2 \mathrm{Wi}^{2}} \sum_{m=1}^{n} \Delta t_{m} \int_{\mathcal{D}} \pi_{h}\left[\operatorname{tr}\left(\beta_{\delta}^{(L)}\left(\boldsymbol{\sigma}_{\alpha, \delta, h}^{(L,) m}\right)+\left[\beta_{\delta}^{(L)}\left(\boldsymbol{\sigma}_{\alpha, \delta, h}^{(L,) m}\right)\right]^{-1}-2 \boldsymbol{I}\right)\right] \\
\leq F_{\delta, h}^{(L)}\left(\boldsymbol{u}_{h}^{0}, \boldsymbol{\sigma}_{h}^{0}\right)+\frac{1+C_{P}}{2(1-\varepsilon)} \sum_{m=1}^{n} \Delta t_{m}\left\|\boldsymbol{f}^{m}\right\|_{H^{-1}(\mathcal{D})}^{2} \leq C .
\end{gathered}
$$

Moreover, it follows that

$$
\begin{array}{r}
\max _{n=0, \ldots, N_{T}} \int_{\mathcal{D}}\left[\left\|\boldsymbol{u}_{\alpha, \delta, h}^{(L,) n}\right\|^{2}+\pi_{h}\left[\left\|\boldsymbol{\sigma}_{\alpha, \delta, h}^{(L,) n}\right\|\right]+\delta^{-1} \pi_{h}\left[\left\|\left[\boldsymbol{\sigma}_{\alpha, \delta, h}^{(L,) n}\right]-\right\|\right]\right] \\
+\sum_{n=1}^{N_{T}} \int_{\mathcal{D}}\left[\Delta t_{n}\left\|\boldsymbol{\nabla} \boldsymbol{u}_{\alpha, \delta, h}^{(L,) n}\right\|^{2}+\Delta t_{n} \pi_{h}\left[\left\|\left[\beta_{\delta}^{(L)}\left(\boldsymbol{\sigma}_{\alpha, \delta, h}^{(L,) n}\right)\right]^{-1}\right\|\right]+\left\|\boldsymbol{u}_{\alpha, \delta, h}^{(L,) n}-\boldsymbol{u}_{\alpha, \delta, h}^{(L,) n-1}\right\|^{2}\right] \\
\leq C .
\end{array}
$$

Proof. Existence and the stability result (5.47) follow immediately from Propositions 5.2 and 5.1. respectively, on noting (5.35), (5.29), (5.30), (3.10a) and (2.19). The bounds (5.48) follow immediately from (5.47), on noting (2.7b), (2.8), (1.7) and the fact that $\beta_{\delta}^{(L)}(\phi) \in \mathbb{R}_{S P D}^{d \times d}$ for any $\phi \in \mathbb{R}_{S}^{d \times d}$.

\subsection{Convergence of $\left(P_{\alpha, \delta, h}^{(L,) \Delta t}\right)$ to $\left(P_{\alpha, h}^{(L,) \Delta t}\right)$}

We now consider the corresponding direct finite element approximation of $\left(\mathrm{P}_{\alpha}^{(L)}\right)$, i.e. $\left(\mathrm{P}_{\alpha, h}^{(L,) \Delta t}\right)$ without the regularization $\delta$ :

We introduce

$$
\mathrm{S}_{h, P D}^{1}=\left\{\phi \in \mathrm{S}_{h}^{1}: \phi\left(P_{p}\right) \in \mathbb{R}_{S P D}^{d \times d} \quad \text { for } \quad p=1, \ldots, N_{P}\right\} \subset \mathrm{S}_{P D} .
$$

It follows from (5.30) that $\boldsymbol{\sigma}_{h}^{0} \in \mathrm{S}_{h, P D}^{1}$.

$$
\left(\mathbf{P}_{\alpha, h}^{(L,) \Delta t}\right) \text { Setting }\left(\boldsymbol{u}_{\alpha, h}^{(L), 0}, \boldsymbol{\sigma}_{\alpha, h}^{(L), 0}\right)=\left(\boldsymbol{u}_{h}^{0}, \boldsymbol{\sigma}_{h}^{0}\right) \in \mathrm{V}_{h}^{1} \times \mathrm{S}_{h, P D}^{1} \text {, then for } n=
$$
$1, \ldots, N_{T}$ find $\left(\boldsymbol{u}_{\alpha, h}^{(L,) n}, \boldsymbol{\sigma}_{\alpha, h}^{(L,) n}\right) \in \mathrm{V}_{h}^{1} \times \mathrm{S}_{h}^{1}$ such that for any test functions $(\boldsymbol{v}, \boldsymbol{\phi}) \in$ 


$$
\begin{aligned}
& \mathrm{V}_{h}^{1} \times \mathrm{S}_{h}^{1} \\
& \int_{\mathcal{D}} {\left[\operatorname{Re}\left(\frac{\boldsymbol{u}_{\alpha, h}^{(L,) n}-\boldsymbol{u}_{\alpha, h}^{(L,) n-1}}{\Delta t_{n}}\right) \cdot \boldsymbol{v}\right.} \\
&+\frac{\operatorname{Re}}{2}\left[\left(\left(\boldsymbol{u}_{\alpha, h}^{(L,) n-1} \cdot \nabla\right) \boldsymbol{u}_{\alpha, h}^{(L,) n}\right) \cdot \boldsymbol{v}-\boldsymbol{u}_{\alpha, h}^{(L,) n} \cdot\left(\left(\boldsymbol{u}_{\alpha, h}^{(L,) n-1} \cdot \nabla\right) \boldsymbol{v}\right)\right] \\
&\left.+(1-\varepsilon) \boldsymbol{\nabla} \boldsymbol{u}_{\alpha, h}^{(L,) n}: \boldsymbol{\nabla} \boldsymbol{v}+\frac{\varepsilon}{\mathrm{Wi}} \pi_{h}\left[\beta^{(L)}\left(\boldsymbol{\sigma}_{\alpha, h}^{(L,) n}\right)\right]: \nabla \boldsymbol{v}\right]=\left\langle\boldsymbol{f}^{n}, \boldsymbol{v}\right\rangle_{H_{0}^{1}(\mathcal{D})}, \quad(5,5) \\
& \int_{\mathcal{D}} \pi_{h}\left[\left(\frac{\boldsymbol{\sigma}_{\alpha, h}^{(L,) n}-\boldsymbol{\sigma}_{\alpha, h}^{(L,) n-1}}{\Delta t_{n}}\right): \boldsymbol{\phi}+\frac{1}{\mathrm{Wi}}\left(\boldsymbol{\sigma}_{\alpha, h}^{(L,) n}-\boldsymbol{I}\right): \boldsymbol{\phi}\right] \\
& \quad+\alpha \int_{D} \boldsymbol{\nabla} \boldsymbol{\sigma}_{\alpha, h}^{(L,) n}:: \boldsymbol{\nabla} \boldsymbol{\phi}-2 \int_{\mathcal{D}} \boldsymbol{\nabla} \boldsymbol{u}_{\alpha, h}^{(L,) n}: \pi_{h}\left[\boldsymbol{\phi} \beta^{(L)}\left(\boldsymbol{\sigma}_{\alpha, h}^{(L,) n}\right)\right] \\
& \quad-\int_{\mathcal{D}} \sum_{m=1}^{d} \sum_{p=1}^{d}\left[\boldsymbol{u}_{\alpha, h}^{(L, h-1}\right]_{m} \Lambda_{m, p}^{(L)}\left(\boldsymbol{\sigma}_{\alpha, h}^{(L,) n}\right): \frac{\partial \boldsymbol{\phi}}{\partial \boldsymbol{x}_{p}}=0
\end{aligned}
$$

Remark 5.1. Due to the presence of $\beta^{(L)}$ in (5.50a b), it is implicitly assumed that $\boldsymbol{\sigma}_{\alpha, h}^{(L,) n} \in \mathrm{S}_{h, P D}^{1}, n=1, \ldots, N_{T}$; recall (2.2). In addition, $\Lambda_{m, p}^{(L)}(\phi)$ for $\phi \in \mathrm{S}_{h, P D}^{1}$ is defined similarly to (5.22) with $\widehat{\Lambda}_{\delta, j}^{(L)}(\widehat{\phi})$ replaced by $\widehat{\Lambda}_{j}^{(L)}(\widehat{\phi})$, which is defined similarly to (5.21a,b) with $\lambda_{\delta, j}^{(L)}, \beta_{\delta}^{(L)}$ and $G_{\delta}^{(L)}$ replaced by $\lambda_{j}^{(L)}, \beta^{(L)}$ and $G^{(L)}$, with $\lambda_{j}^{(L)}$ defined similarly to $\lambda_{\delta, j}^{(L)}$ with $\beta_{\delta}^{(L)}, G_{\delta}^{(L)}$ and $H_{\delta}^{(L)}$ replaced by $\beta^{(L)}, G^{(L)}$ and $H^{(L)}$. Hence, similarly to (5.26), we have that

$$
\left\|\Lambda_{m, p}^{L}(\phi)\right\|_{L^{\infty}(\mathcal{D})} \leq C L \quad \forall \phi \in \mathrm{S}_{h, P D}^{1} .
$$

We introduce also the unregularised free energy

$$
F_{h}^{(L)}(\boldsymbol{v}, \phi):=\frac{\operatorname{Re}}{2} \int_{\mathcal{D}}\|\boldsymbol{v}\|^{2}+\frac{\varepsilon}{2 \mathrm{Wi}} \int_{\mathcal{D}} \pi_{h}\left[\operatorname{tr}\left(\boldsymbol{\phi}-G^{(L)}(\boldsymbol{\phi})-\boldsymbol{I}\right)\right],
$$

which is well defined for all $(\boldsymbol{v}, \boldsymbol{\phi}) \in \mathrm{V}_{h}^{1} \times \mathrm{S}_{h, P D}^{1}$.

Theorem 5.2. For all regular partitionings $\mathcal{T}_{h}$ of $\mathcal{D}$ into simplices $\left\{K_{k}\right\}_{k=1}^{N_{K}}$ and all partitionings $\left\{\Delta t_{n}\right\}_{n=1}^{N_{T}}$ of $[0, T]$, there exists a subsequence $\left\{\left\{\left(\boldsymbol{u}_{\alpha, \delta, h}^{(L,) n}, \boldsymbol{\sigma}_{\alpha, \delta, h}^{(L,) n}\right)\right\}_{n=1}^{N_{T}}\right\}_{\delta>0}$, where $\left\{\left(\boldsymbol{u}_{\alpha, \delta, h}^{(L,) n}, \boldsymbol{\sigma}_{\alpha, \delta, h}^{(L,) n}\right)\right\}_{n=1}^{N_{T}} \in\left[\mathrm{V}_{h}^{1} \times \mathrm{S}_{h}^{1}\right]^{N_{T}}$ solves $\left(P_{\alpha, \delta, h}^{(L,) \Delta t}\right)$, and $\left\{\left(\boldsymbol{u}_{\alpha, h}^{(L,) n}, \boldsymbol{\sigma}_{\alpha, h}^{(L,) n}\right)\right\}_{n=1}^{N_{T}} \in\left[\mathrm{V}_{h}^{1} \times \mathrm{S}_{h}^{1}\right]^{N_{T}}$ such that for the subsequence

$$
\boldsymbol{u}_{\alpha, \delta, h}^{(L,) n} \rightarrow \boldsymbol{u}_{\alpha, h}^{(L,) n}, \quad \boldsymbol{\sigma}_{\alpha, \delta, h}^{(L,) n} \rightarrow \boldsymbol{\sigma}_{\alpha, h}^{(L,) n} \quad \text { as } \delta \rightarrow 0_{+}, \quad \text { for } \quad n=1, \ldots, N_{T} .
$$

In addition, for $n=1, \ldots, N_{T}, \boldsymbol{\sigma}_{\alpha, h}^{(L,) n} \in \mathrm{S}_{h, P D}^{1}$, and $\left\{\left(\boldsymbol{u}_{\alpha, h}^{(L,) n}, \boldsymbol{\sigma}_{\alpha, h}^{(L,) n}\right)\right\}_{n=1}^{N_{T}} \in\left[\mathrm{V}_{h}^{1} \times\right.$ $\left.\mathrm{S}_{h, P D}^{1}\right]^{N_{T}}$ solves $\left(P_{\alpha, h}^{(L,) \Delta t}\right)$.

Moreover, we have for $n=1, \ldots, N_{T}$ that

$$
\frac{F_{h}^{(L)}\left(\boldsymbol{u}_{\alpha, h}^{(L,) n}, \boldsymbol{\sigma}_{\alpha, h}^{(L,) n}\right)-F_{h}^{(L)}\left(\boldsymbol{u}_{\alpha, h}^{(L,) n-1}, \boldsymbol{\sigma}_{\alpha, h}^{(L,) n-1}\right)}{\Delta t_{n}}+\frac{\operatorname{Re}}{2 \Delta t_{n}} \int_{\mathcal{D}}\left\|\boldsymbol{u}_{\alpha, h}^{(L,) n}-\boldsymbol{u}_{\alpha, h}^{(L,) n-1}\right\|^{2}
$$




$$
\begin{aligned}
& +(1-\varepsilon) \int_{\mathcal{D}}\left\|\boldsymbol{\nabla} \boldsymbol{u}_{\alpha, h}^{(L,) n}\right\|^{2}+\frac{\varepsilon}{2 \mathrm{Wi}^{2}} \int_{\mathcal{D}} \pi_{h}\left[\operatorname{tr}\left(\beta^{L}\left(\boldsymbol{\sigma}_{\alpha, h}^{(L,) n}\right)+\left[\beta^{L}\left(\boldsymbol{\sigma}_{\alpha, h}^{(L,) n}\right)\right]^{-1}-2 \boldsymbol{I}\right)\right] \\
& \quad \leq \frac{1}{2}(1-\varepsilon) \int_{\mathcal{D}}\left\|\boldsymbol{\nabla} \boldsymbol{u}_{\alpha, h}^{(L,) n}\right\|^{2}+\frac{1+C_{P}}{2(1-\varepsilon)}\left\|\boldsymbol{f}^{n}\right\|_{H^{-1}(\mathcal{D})}^{2}
\end{aligned}
$$

and

$$
\begin{array}{r}
\max _{n=0, \ldots, N_{T}} \int_{\mathcal{D}}\left[\left\|\boldsymbol{u}_{\alpha, h}^{(L,) n}\right\|^{2}+\pi_{h}\left[\left\|\boldsymbol{\sigma}_{\alpha, h}^{(L,) n}\right\|\right]\right] \\
+\sum_{n=1}^{N_{T}} \int_{\mathcal{D}}\left[\Delta t_{n}\left\|\nabla \boldsymbol{u}_{\alpha, h}^{(L,) n}\right\|^{2}+\Delta t_{n} \pi_{h}\left[\left\|\left[\beta^{(L)}\left(\boldsymbol{\sigma}_{\alpha, h}^{(L,) n}\right)\right]^{-1}\right\|\right]+\left\|\boldsymbol{u}_{\alpha, h}^{(L,) n}-\boldsymbol{u}_{\alpha, h}^{(L,) n-1}\right\|^{2}\right] \\
\leq C .
\end{array}
$$

Proof. For any integer $n \in\left[1, N_{T}\right]$, the desired subsequence convergence result (5.53) follows immediately from (5.48), as $\left(\boldsymbol{u}_{\alpha, \delta, h}^{(L,) n}, \boldsymbol{\sigma}_{\alpha, \delta, h}^{(L,) n}\right)$ are finite dimensional for fixed $\mathrm{V}_{h}^{1} \times S_{h}^{1}$. It also follows from (5.48), (5.53) and (2.17) that $\pi_{h}\left[\left[\boldsymbol{\sigma}_{\alpha, \delta, h}^{(L,) n}\right]_{-}\right]$ vanishes on $\mathcal{D}$, so that $\boldsymbol{\sigma}_{\alpha, h}^{(L,) n}$ must be non-negative definite on $\mathcal{D}$. Hence on noting this, (2.3), (2.17) and (5.53), we have the following subsequence convergence results

$$
\beta_{\delta}^{(L)}\left(\boldsymbol{\sigma}_{\alpha, h}^{(L, h)}\right) \rightarrow \beta^{(L)}\left(\boldsymbol{\sigma}_{\alpha, h}^{(L,) n}\right) \quad \text { and } \quad \beta_{\delta}^{(L)}\left(\boldsymbol{\sigma}_{\alpha, \delta, h}^{(L,) n}\right) \rightarrow \beta^{(L)}\left(\boldsymbol{\sigma}_{\alpha, h}^{(L,) n}\right) \quad \text { as } \quad \delta \rightarrow 0_{+} .
$$

It also follows from (5.48), (5.56) and as $\left[\beta_{\delta}^{(L)}\left(\boldsymbol{\sigma}_{\alpha, \delta, h}^{(L,) n}\right)\right]^{-1} \beta_{\delta}^{(L)}\left(\boldsymbol{\sigma}_{\alpha, \delta, h}^{(L,) n}\right)=\boldsymbol{I}$ that the following subsequence result

$$
\pi_{h}\left[\left[\beta_{\delta}^{(L)}\left(\boldsymbol{\sigma}_{\alpha, \delta, h}^{(L,) n}\right)\right]^{-1}\right] \rightarrow \pi_{h}\left[\left[\beta^{(L)}\left(\boldsymbol{\sigma}_{\alpha, h}^{(L,) n}\right)\right]^{-1}\right] \quad \text { as } \quad \delta \rightarrow 0_{+}
$$

holds, and so $\sigma_{\alpha, h}^{(L,) n} \in \mathrm{S}_{h, P D}^{1}$. Therefore, we have from (5.53) and (2.1) that

$$
G_{\delta}^{(L)}\left(\boldsymbol{\sigma}_{\alpha, \delta, h}^{(L,) n}\right) \rightarrow G^{(L)}\left(\boldsymbol{\sigma}_{\alpha, h}^{(L,) n}\right) \quad \text { as } \quad \delta \rightarrow 0_{+} .
$$

Similarly to (5.58), it follows from (5.53), (5.56), (5.22) and (5.21) b) as $\sigma_{\alpha, h}^{(L,), n} \in$ $\mathrm{S}_{h, P D}^{1}$ that for $m, p=1, \ldots, d$

$$
\Lambda_{\delta, m, p}^{(L)}\left(\boldsymbol{\sigma}_{\alpha, \delta, h}^{(L,) n}\right) \rightarrow \Lambda_{m, p}^{(L)}\left(\boldsymbol{\sigma}_{\alpha, h}^{(L,) n}\right) \quad \text { as } \quad \delta \rightarrow 0_{+} .
$$

Hence using (5.53), (5.56) and (5.59), we can pass to the limit $\delta \rightarrow 0_{+}$in $\left(\mathrm{P}_{\alpha, \delta, h}^{(L,) \Delta t}\right)$, (5.34a b) , to show that $\left\{\left(\boldsymbol{u}_{\alpha, h}^{(L,) n}, \boldsymbol{\sigma}_{\alpha, h}^{(L,) n}\right)\right\}_{n=1}^{N_{T}} \in\left[\mathrm{V}_{h}^{1} \times \mathrm{S}_{h, P D}^{1}\right]^{N_{T}}$ solves $\left(\mathrm{P}_{\alpha, h}^{(L,) \Delta t}\right)$, (5.50a,b). Similarly, using (5.53), (5.56), (5.57) and (5.58), and noting (5.35) and (5.52), we can pass to the limit $\delta \rightarrow 0_{+}$in (5.36) and (5.48) to obtain the desired results (5.54) and (5.55).

For later purposes, we introduce the following notation in line with (3.9). Let $\boldsymbol{u}_{\alpha, h}^{(L,) \Delta t} \in C\left([0, T] ; \mathrm{V}_{h}^{1}\right)$ and $\boldsymbol{u}_{\alpha, h}^{(L,) \Delta t, \pm} \in L^{\infty}\left(0, T ; \mathrm{V}_{h}^{1}\right)$ be such that for $n=1, \ldots, N_{T}$

$$
\boldsymbol{u}_{\alpha, h}^{(L,) \Delta t}(t, \cdot):=\frac{t-t^{n-1}}{\Delta t_{n}} \boldsymbol{u}_{\alpha, h}^{(L,) n}(\cdot)+\frac{t^{n}-t}{\Delta t_{n}} \boldsymbol{u}_{\alpha, h}^{(L,) n-1}(\cdot) \quad t \in\left[t^{n-1}, t^{n}\right],
$$


John W. Barrett and Sébastien Boyaval

$$
\begin{aligned}
& \boldsymbol{u}_{\alpha, h}^{(L,) \Delta t,+}(t, \cdot):=\boldsymbol{u}_{\alpha, h}^{(L,) n}(\cdot), \quad \boldsymbol{u}_{\alpha, h}^{(L,) \Delta t,-}(t, \cdot):=\boldsymbol{u}_{\alpha, h}^{(L,) n-1}(\cdot) \quad t \in\left[t^{n-1}, t^{n}\right) \\
& \text { and } \Delta(t):=\Delta t_{n} \\
& t \in\left[t^{n-1}, t^{n}\right),
\end{aligned}
$$

We note that

$$
\boldsymbol{u}_{\alpha, h}^{(L,) \Delta t}-\boldsymbol{u}_{\alpha, h}^{(L,) \Delta t, \pm}=\left(t-t_{ \pm}^{n}\right) \frac{\partial \boldsymbol{u}_{\alpha, h}^{(L,) \Delta t}}{\partial t} \quad t \in\left(t^{n-1}, t^{n}\right), \quad n=1, \ldots, N_{T},
$$

where $t_{+}^{n}:=t^{n}$ and $t_{-}^{n}:=t^{n-1}$. We define $\boldsymbol{\sigma}_{\alpha, h}^{(L,) \Delta t} \in C\left([0, T] ; \mathrm{S}_{h, P D}^{1}\right)$ and $\boldsymbol{\sigma}_{\alpha, h}^{(L,) \Delta t, \pm} \in L^{\infty}\left(0, T ; \mathrm{S}_{h, P D}^{1}\right)$ similarly to (5.60a, b).

Using the notation (5.60a b), (5.50a) multiplied by $\Delta t_{n}$ and summed for $n=$ $1, \ldots, N_{T}$ can be restated as:

$$
\begin{aligned}
& \int_{0}^{T} \int_{\mathcal{D}}\left[\operatorname{Re} \frac{\partial \boldsymbol{u}_{\alpha, h}^{(L,) \Delta t}}{\partial t} \cdot \boldsymbol{v}+(1-\varepsilon) \nabla \boldsymbol{u}_{\alpha, h}^{(L,) \Delta t,+}: \boldsymbol{\nabla} \boldsymbol{v}\right] d t \\
&+ \frac{\operatorname{Re}}{2} \int_{0}^{T} \int_{\mathcal{D}}\left[\left[\left(\boldsymbol{u}_{\alpha, h}^{(L,) \Delta t,-} \cdot \boldsymbol{\nabla}\right) \boldsymbol{u}_{\alpha, h}^{(L,) \Delta t,+}\right] \cdot \boldsymbol{v}-\left[\left(\boldsymbol{u}_{\alpha, h}^{(L,) \Delta t,-} \cdot \boldsymbol{\nabla}\right) \boldsymbol{v}\right] \cdot \boldsymbol{u}_{\alpha, h}^{(L,) \Delta t,+}\right] d t \\
& \quad=\int_{0}^{T}\left[\left\langle\boldsymbol{f}^{+}, \boldsymbol{v}\right\rangle_{H_{0}^{1}(\mathcal{D})}-\frac{\varepsilon}{\mathrm{Wi}} \int_{\mathcal{D}} \pi_{h}\left[\beta^{(L)}\left(\boldsymbol{\sigma}_{\alpha, h}^{(L,) \Delta t,+}\right)\right]: \boldsymbol{\nabla} \boldsymbol{v}\right] d t \\
& \forall \boldsymbol{v} \in L^{2}\left(0, T ; \mathrm{V}_{h}^{1}\right) .
\end{aligned}
$$

Similarly, (5.50b) multiplied by $\Delta t_{n}$ and summed for $n=1, \ldots, N_{T}$ can be restated as:

$$
\begin{aligned}
& \int_{0}^{T} \int_{\mathcal{D}} \pi_{h}\left[\frac{\partial \boldsymbol{\sigma}_{\alpha, h}^{(L,) \Delta t}}{\partial t}: \chi+\frac{1}{\mathrm{Wi}}\left(\boldsymbol{\sigma}_{\alpha, h}^{(L,) \Delta t,+}-\boldsymbol{I}\right): \chi\right] d t \\
&+\alpha \int_{0}^{T} \int_{\mathcal{D}} \boldsymbol{\nabla} \boldsymbol{\sigma}_{\alpha, h}^{(L,) \Delta t,+}:: \nabla \boldsymbol{\chi} d t-2 \int_{0}^{T} \int_{\mathcal{D}} \boldsymbol{\nabla} \boldsymbol{u}_{\alpha, h}^{(L,) \Delta t,+}: \pi_{h}\left[\boldsymbol{\chi} \beta^{(L)}\left(\boldsymbol{\sigma}_{\alpha, h}^{(L,) \Delta t,+}\right)\right] d t \\
&-\int_{0}^{T} \int_{\mathcal{D}} \sum_{m=1}^{d} \sum_{p=1}^{d}\left[\boldsymbol{u}_{\alpha, h}^{(L,) \Delta t,-}\right]_{m} \Lambda_{m, p}^{(L)}\left(\boldsymbol{\sigma}_{\alpha, h}^{(L,) \Delta t,+}\right): \frac{\partial \boldsymbol{\chi}}{\partial \boldsymbol{x}_{p}} d t=0 \\
& \forall \chi \in L^{2}\left(0, T ; \mathrm{S}_{h}^{1}\right) .
\end{aligned}
$$

We note also the following Lemma for later purposes.

Lemma 5.3. For all $K_{k} \in \mathcal{T}_{h}$, and for all $\phi \in \mathrm{S}_{h, P D}^{1}$ we have that

$$
\begin{gathered}
\int_{K_{k}}\left\|\pi_{h}\left[\beta^{(L)}(\phi)\right]-\beta^{(L)}(\phi)\right\|^{2}+\max _{m, p=1, \ldots, d} \int_{K_{k}}\left\|\Lambda_{m, p}^{(L)}(\phi)-\beta^{(L)}(\phi) \delta_{m p}\right\|^{2} \\
\leq C h^{2} \int_{K_{k}}\|\nabla \phi\|^{2} .
\end{gathered}
$$

Proof. First, we have from (2.17) that for all $\phi \in \mathrm{S}_{h, P D}^{1}$

$$
\int_{K_{k}}\left\|\pi_{h}\left[\beta^{(L)}(\phi)\right]-\beta^{(L)}(\phi)\right\|^{2} \leq C\left|K_{k}\right| \sum_{j=0}^{d}\left\|\beta^{(L)}\left(\phi\left(P_{j}^{k}\right)\right)-\beta^{(L)}(\phi)\right\|_{L^{\infty}\left(K_{k}\right)}^{2}
$$




$$
\begin{aligned}
& \leq C\left|K_{k}\right| \sum_{j=0}^{d}\left\|\phi\left(P_{j}^{k}\right)-\phi\right\|_{L^{\infty}\left(K_{k}\right)}^{2} \\
& \leq C h^{2}\left|K_{k}\right|\|\nabla \phi\|_{L^{\infty}\left(K_{k}\right)}^{2} \leq C h^{2} \int_{K_{k}}\|\nabla \phi\|^{2} .
\end{aligned}
$$

where $\left\{P_{j}^{k}\right\}_{j=0}^{d}$ are the vertices of $K_{k}$. Hence we have the desired first bound in (5.64).

It follows from the $\delta$ independent versions of (5.22) and (5.21a,b), recall Remark 5.1. (5.25) and (2.17) that for all $\phi \in \mathrm{S}_{h, P D}^{1}$

$$
\begin{aligned}
\int_{K_{k}} \| \Lambda_{m, p}^{(L)}(\boldsymbol{\phi})- & \pi_{h}\left[\beta^{(L)}(\phi)\right] \delta_{m p} \|^{2} \\
& =\int_{K_{k}}\left\|\sum_{j=1}^{d}\left[\left[\left(B_{k}^{T}\right)^{-1}\right]_{m j}\left[\widehat{\Lambda}_{j}^{(L)}(\widehat{\phi})-\pi_{h}\left[\beta^{(L)}(\boldsymbol{\phi})\right]\right]\left[B_{k}^{T}\right]_{j p}\right]\right\| \\
& \leq C \int_{K_{k}} \sum_{j=1}^{d}\left\|\widehat{\Lambda}_{j}^{(L)}(\widehat{\phi})-\pi_{h}\left[\beta^{(L)}(\boldsymbol{\phi})\right]\right\|^{2} \\
& \leq C\left|K_{k}\right| \max _{i, j=0, \ldots, d}\left\|\beta^{(L)}\left(\phi\left(P_{j}^{k}\right)\right)-\beta^{(L)}\left(\phi\left(P_{i}^{k}\right)\right)\right\|^{2} \\
& \leq C\left|K_{k}\right| \max _{i, j=0, \ldots, d}\left\|\phi\left(P_{j}^{k}\right)-\phi\left(P_{i}^{k}\right)\right\|^{2} \\
& \leq C h^{2} \int_{K_{k}}\|\nabla \phi\|^{2} .
\end{aligned}
$$

Combining (5.66) and the first bound in (5.64) yields the second bound in (5.64) $\square$

\section{Convergence of $\left(\mathrm{P}_{\alpha, h}^{L, \Delta t}\right)$ to $\left(\mathrm{P}_{\alpha}^{L}\right)$}

Before proving our convergence result, we first deduce some simple inequalities

that will be required. We recall the following well-known results concerning the interpolant $\pi_{h}$ :

$$
\begin{aligned}
\left\|\left(\boldsymbol{I}-\pi_{h}\right) \boldsymbol{\phi}\right\|_{W^{1, \infty}\left(K_{k}\right)} \leq C h|\phi|_{W^{2, \infty}\left(K_{k}\right)} \quad \forall \phi \in\left[W^{2, \infty}\left(K_{k}\right)\right]_{S}^{d \times d}, & k=1, \ldots, N_{K} ; \\
\left\|\left(\boldsymbol{I}-\pi_{h}\right)[\chi: \phi]\right\|_{L^{2}(\mathcal{D})} & \leq C h^{2}\|\nabla \chi\|_{L^{2}(\mathcal{D})}\|\nabla \phi\|_{L^{\infty}(\mathcal{D})} \\
& \leq C h\|\chi\|_{L^{2}(\mathcal{D})}\|\nabla \phi\|_{L^{\infty}(\mathcal{D})} \quad \forall \boldsymbol{\chi}, \boldsymbol{\phi} \in \mathrm{S}_{h}^{1} .
\end{aligned}
$$

We note for any $\zeta \in \mathbb{R}_{>0}$ that

$$
\begin{aligned}
& {\left[\pi_{h}[\chi: \phi]\right](\boldsymbol{x}) \leq \frac{1}{2}\left[\pi_{h}\left[\zeta\|\boldsymbol{\chi}\|^{2}+\zeta^{-1}\|\boldsymbol{\phi}\|^{2}\right]\right](\boldsymbol{x}) } \\
& \forall \boldsymbol{x} \in K_{k}, \quad \forall \boldsymbol{\chi}, \boldsymbol{\phi} \in\left[C\left(\overline{K_{k}}\right)\right]^{d \times d}, \quad k=1, \ldots, N_{K} .
\end{aligned}
$$


Combining (5.12), (1.4b) and (2.4), we have for all $\phi \in \mathrm{S}_{h, P D}^{1}$ and for all $\boldsymbol{\psi} \in \mathrm{S}_{h}^{1}$ that

$$
\begin{aligned}
\int_{D}\left\|\pi_{h}\left[\boldsymbol{\psi} \beta^{L}(\boldsymbol{\phi})\right]\right\|^{2} & \leq \int_{D} \pi_{h}\left[\left\|\boldsymbol{\psi} \beta^{L}(\boldsymbol{\phi})\right\|^{2}\right] \leq \int_{D} \pi_{h}\left[\|\boldsymbol{\psi}\|^{2}\left\|\beta^{L}(\boldsymbol{\phi})\right\|^{2}\right] \\
& \leq d L^{2} \int_{D} \pi_{h}\left[\|\boldsymbol{\psi}\|^{2}\right] .
\end{aligned}
$$

We require also the $L^{2}$ projector $\mathcal{R}_{h}: \mathrm{V} \rightarrow \mathrm{V}_{h}^{1}$ defined by

$$
\int_{\mathcal{D}}\left(\boldsymbol{v}-\mathcal{R}_{h} \boldsymbol{v}\right) \boldsymbol{w}=0 \quad \forall \boldsymbol{w} \in \mathrm{V}_{h}^{1} .
$$

In addition, we require $\mathcal{P}_{h}: \mathrm{S} \rightarrow \mathrm{S}_{h}^{1}$ defined by

$$
\int_{\mathcal{D}} \pi_{h}\left[\mathcal{P}_{h} \chi: \phi\right]=\int_{\mathcal{D}} \chi: \phi \quad \forall \phi \in \mathrm{S}_{h}^{1} .
$$

It is easily deduced for $p=1, \ldots, N_{P}$ and $i, j=1, \ldots, d$ that

$$
\left[\mathcal{P}_{h} \boldsymbol{\chi}\right]_{i j}\left(P_{p}\right)=\frac{1}{\int_{\mathcal{D}} \eta_{p}} \int_{\mathcal{D}}\left[\mathcal{P}_{h} \boldsymbol{\chi}\right]_{i j} \eta_{p}
$$

where $\eta_{p} \in \mathrm{Q}_{h}^{1}$ is such that $\eta_{p}\left(P_{r}\right)=\delta_{p r}$ for $p, r=1, \ldots, N_{P}$. It follows from (6.5) and (5.12) with $\phi=\mathcal{P}_{h} \chi$, in both cases, that

$$
\int_{\mathcal{D}}\left\|\mathcal{P}_{h} \chi\right\|^{2} \leq \int_{\mathcal{D}} \pi_{h}\left[\left\|\mathcal{P}_{h} \chi\right\|^{2}\right] \leq \int_{\mathcal{D}}\|\chi\|^{2} \quad \forall \chi \in\left[L^{2}(\mathcal{D})\right]_{S}^{d \times d} .
$$

We shall assume from now on that $\mathcal{D}$ is convex and that the family $\left\{\mathcal{T}_{h}\right\}_{h>0}$ is quasi-uniform, i.e. $h_{k} \geq C h, k=1, \ldots, N_{K}$. It then follows that

$$
\left\|\mathcal{R}_{h} \boldsymbol{v}\right\|_{H^{1}(\mathcal{D})} \leq C\|\boldsymbol{v}\|_{H^{1}(\mathcal{D})} \quad \forall \boldsymbol{v} \in \mathrm{V}
$$

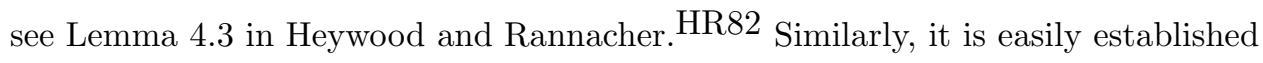
that

$$
\left\|\mathcal{P}_{h} \chi\right\|_{H^{1}(\mathcal{D})} \leq C\|\chi\|_{H^{1}(\mathcal{D})} \quad \forall \chi \in\left[H^{1}(\mathcal{D})\right]_{S}^{d \times d} .
$$

Let $\left(\left[H^{1}(\mathcal{D})\right]_{S}^{d \times d}\right)^{\prime}$ be the topological dual of $\left[H^{1}(\mathcal{D})\right]_{S}^{d \times d}$ with $\left[L^{2}(\mathcal{D})\right]_{S}^{d \times d}$ being the pivot space. Let $\mathcal{E}:\left(\left[H^{1}(\mathcal{D})\right]_{S}^{d \times d}\right)^{\prime} \rightarrow\left[H^{1}(\mathcal{D})\right]_{S}^{d \times d}$ be such that $\mathcal{E} \chi$ is the unique solution of the Helmholtz problem

$$
\int_{\mathcal{D}}[\boldsymbol{\nabla}(\mathcal{E} \chi):: \nabla \phi+(\mathcal{E} \chi): \phi]=\langle\chi, \phi\rangle_{H^{1}(\mathcal{D})} \quad \forall \phi \in\left[H^{1}(\mathcal{D})\right]_{S}^{d \times d},
$$

where $\langle\cdot, \cdot\rangle_{H^{1}(\mathcal{D})}$ denotes the duality pairing between $\left(\left[H^{1}(\mathcal{D})\right]_{S}^{d \times d}\right)^{\prime}$ and $\left[H^{1}(\mathcal{D})\right]_{S}^{d \times d}$. We note that

$$
\langle\chi, \mathcal{E} \chi\rangle_{H^{1}(\mathcal{D})}=\|\mathcal{E} \boldsymbol{\chi}\|_{H^{1}(\mathcal{D})}^{2} \quad \forall \chi \in\left(\left[H^{1}(\mathcal{D})\right]_{S}^{d \times d}\right)^{\prime},
$$

and $\|\mathcal{E} \cdot\|_{H^{1}(\mathcal{D})}$ is a norm on $\left(\left[H^{1}(\mathcal{D})\right]_{S}^{d \times d}\right)^{\prime}$. 
Let $\mathrm{V}^{\prime}$ be the topological dual of $\mathrm{V}$ with the space of weakly divergent free functions in $\left[L^{2}(\mathcal{D})\right]^{d}$ being the pivot space. Let $\mathcal{S}: \mathrm{V}^{\prime} \rightarrow \mathrm{V}$ be such that $\mathcal{S} \boldsymbol{w}$ is the unique solution to the Helmholtz-Stokes problem

$$
\int_{\mathcal{D}}[\boldsymbol{\nabla}(\mathcal{S} \boldsymbol{w}): \boldsymbol{\nabla} \boldsymbol{v}+(\mathcal{S} \boldsymbol{w}) \cdot \boldsymbol{v}]=\langle\boldsymbol{w}, \boldsymbol{v}\rangle_{\mathrm{V}} \quad \forall \boldsymbol{v} \in \mathrm{V},
$$

where $\langle\cdot, \cdot\rangle_{\mathrm{V}}$ denotes the duality pairing between $\mathrm{V}^{\prime}$ and $\mathrm{V}$. We note that

$$
\langle\boldsymbol{w}, \mathcal{S} \boldsymbol{w}\rangle_{\mathrm{V}}=\|\mathcal{S} \boldsymbol{w}\|_{H^{1}(\mathcal{D})}^{2} \quad \forall \boldsymbol{w} \in \mathrm{V}^{\prime},
$$

and $\|\mathcal{S} \cdot\|_{H^{1}(\mathcal{D})}$ is a norm on the reflexive space $\mathrm{V}^{\prime}$. As $\mathrm{V}$ is continuously embedded in $\left[H_{0}^{1}(\mathcal{D})\right]^{d}$, it follows that $\left[H^{-1}(\mathcal{D})\right]^{d}$ is continuously embedded in $\mathrm{V}^{\prime}$.

We recall the following well-known Gagliardo-Nirenberg inequality. Let $r \in$ $[2, \infty)$ if $d=2$, and $r \in[2,6]$ if $d=3$ and $\theta=d\left(\frac{1}{2}-\frac{1}{r}\right)$. Then, there exists a positive constant $C(\mathcal{D}, r, d)$ such that

$$
\|\eta\|_{L^{r}(\mathcal{D})} \leq C(\mathcal{D}, r, d)\|\eta\|_{L^{2}(\mathcal{D})}^{1-\theta}\|\eta\|_{H^{1}(\mathcal{D})}^{\theta} \quad \forall \eta \in H^{1}(\mathcal{D}) .
$$

We recall also the following compactness result, see e.g. Theorem 2.1 on p184 in Temam Tem84 and Simon. Sim87 Let $\mathcal{Y}_{0}, \mathcal{Y}$ and $\mathcal{Y}_{1}$ be Banach spaces, $\mathcal{Y}_{i}, i=0,1$, reflexive, with a compact embedding $\mathcal{Y}_{0} \hookrightarrow \mathcal{Y}$ and a continuous embedding $\mathcal{Y} \hookrightarrow \mathcal{Y}_{1}$. Then, for $\mu_{i}>1, i=0,1$, the following embedding is compact :

$$
\left\{\eta \in L^{\mu_{0}}\left(0, T ; \mathcal{Y}_{0}\right): \frac{\partial \eta}{\partial t} \in L^{\mu_{1}}\left(0, T ; \mathcal{Y}_{1}\right)\right\} \hookrightarrow L^{\mu_{0}}(0, T ; \mathcal{Y}) \text {. }
$$

Theorem 6.1. Under the assumptions of Theorem 5.2, there exists a solution $\left\{\left(\boldsymbol{u}_{\alpha, h}^{L, n}, \boldsymbol{\sigma}_{\alpha, h}^{L, n}\right)\right\}_{n=1}^{N_{T}} \in\left[\mathrm{V}_{h}^{1} \times \mathrm{S}_{h, P D}^{1}\right]^{N_{T}}$ of $\left(P_{\alpha, h}^{L, \Delta t}\right)$ such that, in addition to the bounds (5.54) and (5.55), the following bounds hold:

$$
\begin{gathered}
\max _{n=0, \ldots, N_{T}} \int_{\mathcal{D}} \pi_{h}\left[\left\|\boldsymbol{\sigma}_{\alpha, h}^{L, n}\right\|^{2}\right]+\sum_{n=1}^{N_{T}} \int_{\mathcal{D}}\left[\Delta t_{n} \alpha\left\|\nabla \boldsymbol{\sigma}_{\alpha, h}^{L, n}\right\|^{2}+\pi_{h}\left[\left\|\boldsymbol{\sigma}_{\alpha, h}^{L, n}-\boldsymbol{\sigma}_{\alpha, h}^{L, n-1}\right\|^{2}\right]\right] \\
\leq C(L), \\
\sum_{n=1}^{N_{T}} \Delta t_{n}\left\|\mathcal{S}\left(\frac{\boldsymbol{u}_{\alpha, h}^{L, n}-\boldsymbol{u}_{\alpha, h}^{L, n-1}}{\Delta t_{n}}\right)\right\|_{H^{1}(\mathcal{D})}^{\frac{4}{\vartheta}}+\sum_{n=1}^{N_{T}} \Delta t_{n}\left\|\mathcal{E}\left(\frac{\boldsymbol{\sigma}_{\alpha, h}^{L, n}-\boldsymbol{\sigma}_{\alpha, h}^{L, n-1}}{\Delta t_{n}}\right)\right\|_{H^{1}(\mathcal{D})}^{2} \\
\leq C(L, T) ;
\end{gathered}
$$

where

$$
\vartheta \in(2,4) \quad \text { if } d=2 \quad \text { and } \quad \vartheta=3 \quad \text { if } d=3 .
$$

Proof. Existence and the bounds (5.54) and (5.55) were proved in Theorem 5.2. On choosing $\phi \equiv \sigma_{\alpha, h}^{L, n}$ in the version of (5.50b) dependent on $L$, it follows from (3.14), (6.2), (6.3), (5.55) and (5.51) on applying a Youngs' inequality that

$$
\frac{1}{2} \int_{\mathcal{D}} \pi_{h}\left[\left\|\boldsymbol{\sigma}_{\alpha, h}^{L, n}\right\|^{2}+\left\|\boldsymbol{\sigma}_{\alpha, h}^{L, n}-\boldsymbol{\sigma}_{\alpha, h}^{L, n-1}\right\|^{2}\right]
$$


John W. Barrett and Sébastien Boyaval

$$
\begin{aligned}
+ & \Delta t_{n} \alpha \int_{\mathcal{D}}\left\|\boldsymbol{\nabla} \boldsymbol{\sigma}_{\alpha, h}^{L, n}\right\|^{2}+\frac{\Delta t_{n}}{2 \mathrm{Wi}} \int_{\mathcal{D}} \pi_{h}\left[\left\|\boldsymbol{\sigma}_{\alpha, h}^{L, n}\right\|^{2}\right] \\
\leq & \frac{1}{2} \int_{\mathcal{D}} \pi_{h}\left[\left\|\boldsymbol{\sigma}_{\alpha, h}^{L, n-1}\right\|^{2}\right]+2 \Delta t_{n} \int_{\mathcal{D}}\left\|\nabla \boldsymbol{u}_{\alpha, h}^{L, n}\right\|\left\|\pi_{h}\left[\boldsymbol{\sigma}_{\alpha, h}^{L, n} \beta^{L}\left(\boldsymbol{\sigma}_{\alpha, h}^{L, n}\right)\right]\right\| \\
& +\Delta t_{n} \int_{\mathcal{D}}\left\|\boldsymbol{u}_{\alpha, h}^{L, n-1}\right\|\left\|\nabla \boldsymbol{\sigma}_{\alpha, h}^{L, n}\right\|\left(\sum_{m=1}^{d} \sum_{p=1}^{d}\left\|\Lambda_{m, p}^{L}\left(\boldsymbol{\sigma}_{\alpha, h}^{L, n}\right)\right\|^{2}\right)^{\frac{1}{2}}+\frac{\Delta t_{n} d|D|}{2 \mathrm{Wi}} \\
\leq \frac{1}{2} & {\left[\int_{\mathcal{D}} \pi_{h}\left[\left\|\boldsymbol{\sigma}_{\alpha, h}^{L, n-1}\right\|^{2}\right]+\Delta t_{n} \alpha \int_{\mathcal{D}}\left\|\nabla \boldsymbol{\sigma}_{\alpha, h}^{L, n}\right\|^{2}\right]+\frac{\Delta t_{n}}{4 \mathrm{Wi}} \int_{\mathcal{D}} \pi_{h}\left[\left\|\boldsymbol{\sigma}_{\alpha, h}^{L, n}\right\|^{2}\right] } \\
& +\Delta t_{n} C(L)\left[1+\int_{\mathcal{D}}\left\|\boldsymbol{\nabla} \boldsymbol{u}_{\alpha, h}^{L, n}\right\|^{2}\right] .
\end{aligned}
$$

Hence, summing (6.18) from $n=1, \ldots, m$ for $m=1, \ldots, N_{T}$ yields, on noting (5.55), the desired result (6.16a).

On choosing $\boldsymbol{w}=\mathcal{R}_{h}\left[\mathcal{S}\left(\frac{\boldsymbol{u}_{\alpha, h}^{L, n}-\boldsymbol{u}_{\alpha, h}^{L, n-1}}{\Delta t_{n}}\right)\right] \in \mathrm{V}_{h}^{1}$ in the version of (5.50a) dependent on $L$ yields, on noting (6.4), (6.13), (6.8) and Sobolev embedding, that

$$
\begin{gathered}
\operatorname{Re}\left\|\mathcal{S}\left(\frac{\boldsymbol{u}_{\alpha, h}^{L, n}-\boldsymbol{u}_{\alpha, h}^{L, n-1}}{\Delta t_{n}}\right)\right\|_{H^{1}(\mathcal{D})}^{2} \\
=\operatorname{Re} \int_{\mathcal{D}} \frac{\boldsymbol{u}_{\alpha, h}^{L, n}-\boldsymbol{u}_{\alpha, h}^{L, n-1}}{\Delta t_{n}} \cdot \mathcal{R}_{h}\left[\mathcal{S}\left(\frac{\boldsymbol{u}_{\alpha, h}^{L, n}-\boldsymbol{u}_{\alpha, h}^{L, n-1}}{\Delta t_{n}}\right)\right] \\
=-\int_{\mathcal{D}}\left[(1-\varepsilon) \boldsymbol{\nabla} \boldsymbol{u}_{\alpha, h}^{L, n}+\frac{\varepsilon}{\mathrm{Wi}} \pi_{h}\left[\beta^{L}\left(\boldsymbol{\sigma}_{\alpha, h}^{L, n}\right)\right]\right]: \nabla\left[\mathcal{R}_{h}\left[\mathcal{S}\left(\frac{\boldsymbol{u}_{\alpha, h}^{L, n}-\boldsymbol{u}_{\alpha, h}^{L, n-1}}{\Delta t_{n}}\right)\right]\right] \\
-\frac{\operatorname{Re}}{2} \int_{\mathcal{D}}\left(\left(\boldsymbol{u}_{\alpha, h}^{L, n-1} \cdot \boldsymbol{\nabla}\right) \boldsymbol{u}_{\alpha, h}^{L, n}\right) \cdot \mathcal{R}_{h}\left[\mathcal{S}\left(\frac{\boldsymbol{u}_{\alpha, h}^{L, n}-\boldsymbol{u}_{\alpha, h}^{L, n-1}}{\Delta t_{n}}\right)\right] \\
+\frac{\operatorname{Re}}{2} \int_{\mathcal{D}} \boldsymbol{u}_{\alpha, h}^{L, n} \cdot\left(\left(\boldsymbol{u}_{\alpha, h}^{L, n-1} \cdot \boldsymbol{\nabla}\right)\left[\mathcal{R}_{h}\left[\mathcal{S}\left(\frac{\boldsymbol{u}_{\alpha, h}^{L, n}-\boldsymbol{u}_{\alpha, h}^{L, n-1}}{\Delta t_{n}}\right)\right]\right]\right) \\
+\left\langle\boldsymbol{f}^{n}, \mathcal{R}_{h}\left[\mathcal{S}\left(\frac{\boldsymbol{u}_{\alpha, h}^{L, n}-\boldsymbol{u}_{\alpha, h}^{L, n-1}}{\Delta t_{n}}\right)\right]\right\rangle_{H_{0}^{1}(\mathcal{D})} \\
\leq C\left[\left\|\pi_{h}\left[\beta^{L}\left(\boldsymbol{\sigma}_{\alpha, h}^{L, n}\right)\right]\right\|_{L^{2}(\mathcal{D})}^{2}+\left\|\nabla \boldsymbol{u}_{\alpha, h}^{L, n}\right\|_{L^{2}(\mathcal{D})}^{2}+\|\| \boldsymbol{u}_{\alpha, h}^{L, n-1}\|\| \boldsymbol{u}_{\alpha, h}^{L, n}\|\|_{L^{2}(\mathcal{D})}^{2}\right. \\
\left.+\|\| \boldsymbol{u}_{\alpha, h}^{L, n-1}\|\| \boldsymbol{\nabla} \boldsymbol{u}_{\alpha, h}^{L, n}\|\|_{L^{1+\theta}(\mathcal{D})}^{2}+\left\|\boldsymbol{f}^{n}\right\|_{H^{-1}(\mathcal{D})}^{2}\right],
\end{gathered}
$$

for any $\theta>0$ if $d=2$ and for $\theta=\frac{1}{5}$ if $d=3$. Applying the Cauchy-Schwarz and the algebraic-geometric mean inequalities, in conjunction with (6.14) and the Poincaré inequality (1.8) yields that

$$
\|\| \boldsymbol{u}_{\alpha, h}^{L, n-1}\|\| \boldsymbol{u}_{\alpha, h}^{L, n}\|\|_{L^{2}(\mathcal{D})}^{2} \leq\left\|\boldsymbol{u}_{\alpha, h}^{L, n-1}\right\|_{L^{4}(\mathcal{D})}^{2}\left\|\boldsymbol{u}_{\alpha, h}^{L, n}\right\|_{L^{4}(\mathcal{D})}^{2} \leq \frac{1}{2} \sum_{m=n-1}^{n}\left\|\boldsymbol{u}_{\alpha, h}^{L, m}\right\|_{L^{4}(\mathcal{D})}^{4}
$$




$$
\leq C \sum_{m=n-1}^{n}\left[\left\|\boldsymbol{u}_{\alpha, h}^{L, m}\right\|_{L^{2}(\mathcal{D})}^{4-d}\left\|\nabla \boldsymbol{u}_{\alpha, h}^{L, m}\right\|_{L^{2}(\mathcal{D})}^{d}\right] .
$$

Similarly, we have for any $\theta \in(0,1)$, if $d=2$, that

$$
\begin{aligned}
\|\| \boldsymbol{u}_{\alpha, h}^{L, n-1}\|\| \boldsymbol{\nabla} \boldsymbol{u}_{\alpha, h}^{L, n}\|\|_{L^{1+\theta}(\mathcal{D})}^{2} & \leq\left\|\boldsymbol{u}_{\alpha, h}^{L, n-1}\right\|_{L^{\frac{2(1+\theta)}{1-\theta}}(\mathcal{D})}^{2}\left\|\boldsymbol{\nabla} \boldsymbol{u}_{\alpha, h}^{L, n}\right\|_{L^{2}(\mathcal{D})}^{2} \\
& \leq C\left\|\boldsymbol{u}_{\alpha, h}^{L, n-1}\right\|_{L^{2}(\mathcal{D})}^{\frac{2(1-\theta)}{1+\theta}} \sum_{m=n-1}^{n}\left\|\nabla \boldsymbol{u}_{\alpha, h}^{L, m}\right\|_{L^{2}(\mathcal{D})}^{\frac{2(1+3 \theta)}{1+\theta}} ;
\end{aligned}
$$

and if $d=3,\left(\theta=\frac{1}{5}\right)$, that

$$
\begin{aligned}
\|\| \boldsymbol{u}_{\alpha, h}^{L, n-1}\|\| \nabla \boldsymbol{u}_{\alpha, h}^{L, n}\|\|_{L^{\frac{6}{5}}(\mathcal{D})}^{2} & \leq\left\|\boldsymbol{u}_{\alpha, h}^{L, n-1}\right\|_{L^{3}(\mathcal{D})}^{2}\left\|\nabla \boldsymbol{u}_{\alpha, h}^{L, n}\right\|_{L^{2}(\mathcal{D})}^{2} \\
& \leq C\left\|\boldsymbol{u}_{\alpha, h}^{L, n-1}\right\|_{L^{2}(\mathcal{D})} \sum_{m=n-1}^{n}\left\|\boldsymbol{\nabla} \boldsymbol{u}_{\alpha, h}^{L, m}\right\|_{L^{2}(\mathcal{D})}^{3} .
\end{aligned}
$$

On taking the $\frac{2}{\vartheta}$ power of both sides of (6.19), recall (6.17), multiplying by $\Delta t_{n}$, summing from $n=1, \ldots, N_{T}$ and noting (6.20), (6.21a) with $\theta=\frac{\vartheta-2}{6-\vartheta} \Leftrightarrow \vartheta=$ $\frac{2(1+3 \theta)}{(1+\theta)},(6.21 \mathrm{~b}),(5.27),(3.10 \mathrm{a}),(5.55)$, (5.29) and (2.4) yields that

$$
\begin{aligned}
& \sum_{n=1}^{N_{T}} \Delta t_{n}\left\|\mathcal{S}\left(\frac{\boldsymbol{u}_{\alpha, h}^{L, n}-\boldsymbol{u}_{\alpha, h}^{L, n-1}}{\Delta t_{n}}\right)\right\|_{H^{1}(\mathcal{D})}^{\frac{4}{\vartheta}} \\
& \leq C L^{2}+C(T)\left[\sum_{n=1}^{N_{T}} \Delta t_{n}\left[\left\|\boldsymbol{\nabla} \boldsymbol{u}_{\alpha, h}^{L, n}\right\|_{L^{2}(\mathcal{D})}^{2}+\left\|\boldsymbol{f}^{n}\right\|_{H^{-1}(\mathcal{D})}^{2}\right]\right]^{\frac{2}{\vartheta}} \\
&+C\left[1+\max _{n=0, \ldots, N_{T}}\left(\left\|\boldsymbol{u}_{\alpha, h}^{L, n}\right\|_{L^{2}(\mathcal{D})}^{2}\right)\right]\left[\sum_{n=0}^{N_{T}} \Delta t_{n}\left\|\nabla \boldsymbol{u}_{\alpha, h}^{L, n}\right\|_{L^{2}(\mathcal{D})}^{2}\right] \\
& \leq C(L, T) ;
\end{aligned}
$$

and hence the first bound in 6.16b).

On choosing $\phi=\mathcal{P}_{h}\left[\mathcal{E}\left(\frac{\boldsymbol{\sigma}_{\alpha, h}^{L, n}-\boldsymbol{\sigma}_{\alpha, h}^{L, n-1}}{\Delta t_{n}}\right)\right] \in \mathrm{S}_{h}^{1}$ in the version of (5.50b) dependent on $L$ yields, on noting (6.5), (6.10), (6.2), (6.7), (6.9), (6.3) and (5.51), that

$$
\begin{aligned}
\| \mathcal{E}( & \left.\frac{\boldsymbol{\sigma}_{\alpha, h}^{L, n}-\boldsymbol{\sigma}_{\alpha, h}^{L, n-1}}{\Delta t_{n}}\right) \|_{H^{1}(\mathcal{D})}^{2} \\
& =\int_{\mathcal{D}} \pi_{h}\left[\left(\frac{\boldsymbol{\sigma}_{\alpha, h}^{L, n}-\boldsymbol{\sigma}_{\alpha, h}^{L, n-1}}{\Delta t_{n}}\right): \mathcal{P}_{h}\left[\mathcal{E}\left(\frac{\boldsymbol{\sigma}_{\alpha, h}^{L, n}-\boldsymbol{\sigma}_{\alpha, h}^{L, n-1}}{\Delta t_{n}}\right)\right]\right] \\
& =\frac{1}{\mathrm{Wi}} \int_{\mathcal{D}} \pi_{h}\left[\left(\boldsymbol{I}-\boldsymbol{\sigma}_{\alpha, h}^{L, n}\right): \mathcal{P}_{h}\left[\mathcal{E}\left(\frac{\boldsymbol{\sigma}_{\alpha, h}^{L, n}-\boldsymbol{\sigma}_{\alpha, h}^{L, n-1}}{\Delta t_{n}}\right)\right]\right]
\end{aligned}
$$




$$
\begin{aligned}
& \quad-\alpha \int_{\mathcal{D}} \boldsymbol{\nabla} \boldsymbol{\sigma}_{\alpha, h}^{L, n}:: \boldsymbol{\nabla}\left[\mathcal{P}_{h}\left[\mathcal{E}\left(\frac{\boldsymbol{\sigma}_{\alpha, h}^{L, n}-\boldsymbol{\sigma}_{\alpha, h}^{L, n-1}}{\Delta t_{n}}\right)\right]\right] \\
& \quad+2 \int_{\mathcal{D}} \boldsymbol{\nabla} \boldsymbol{u}_{\alpha, h}^{L, n}: \pi_{h}\left[\mathcal{P}_{h}\left[\mathcal{E}\left(\frac{\boldsymbol{\sigma}_{\alpha, h}^{L, n}-\boldsymbol{\sigma}_{\alpha, h}^{L, n-1}}{\Delta t_{n}}\right)\right] \beta^{L}\left(\boldsymbol{\sigma}_{\alpha, h}^{L, n}\right)\right] \\
& \quad+\int_{\mathcal{D}} \sum_{m=1}^{d} \sum_{p=1}^{d}\left[\boldsymbol{u}_{\alpha, h}^{L, n-1}\right]_{m} \Lambda_{m, p}^{L}\left(\boldsymbol{\sigma}_{\alpha, h}^{L, n}\right): \frac{\partial}{\partial \boldsymbol{x}_{p}}\left[\mathcal{P}_{h}\left[\mathcal{E}\left(\frac{\boldsymbol{\sigma}_{\alpha, h}^{L, n}-\boldsymbol{\sigma}_{\alpha, h}^{L, n-1}}{\Delta t_{n}}\right)\right]\right] \\
& \leq C \int_{\mathcal{D}} \pi_{h}\left[\left\|\boldsymbol{\sigma}_{\alpha, h}^{L, n}\right\|^{2}\right] \\
& +C(L)\left[1+\alpha\left\|\nabla \boldsymbol{\sigma}_{\alpha, h}^{L, n}\right\|_{L^{2}(\mathcal{D})}^{2}+\left\|\boldsymbol{\nabla} \boldsymbol{u}_{\alpha, h}^{L, n}\right\|_{L^{2}(\mathcal{D})}^{2}+\left\|\boldsymbol{u}_{\alpha, h}^{L, n-1}\right\|_{L^{2}(\mathcal{D})}^{2}\right] .
\end{aligned}
$$

Multiplying (6.23) by $\Delta t_{n}$, summing from $n=1, \ldots, N_{T}$ and noting (5.55) and (6.16a) yields the second bound in (6.16b).

\subsection{Convergence of the discrete solutions}

First we note the following result.

Lemma 6.1. For $k=1, \ldots, N_{K}$, it follows that

$$
\int_{K_{k}}\left\|\chi^{-1}\right\| \leq C \int_{K_{k}} \pi_{h}\left[\left\|\chi^{-1}\right\|\right] \quad \forall \chi \in \mathrm{S}_{h, P D}^{1} .
$$

Proof. We recall the well-known result about equivalence of norms

$$
\frac{1}{d^{\frac{1}{2}}}\|\phi\| \leq\|\phi\|_{2}:=\sup _{\boldsymbol{v} \in \mathbb{R}^{d},\|\boldsymbol{v}\|=1}\|\boldsymbol{\phi} \boldsymbol{v}\| \leq\|\phi\| \quad \forall \phi \in \mathbb{R}^{d \times d} .
$$

We recall also that if $\phi \in \mathbb{R}_{S P D}^{d \times d}$, then

$$
\boldsymbol{z}^{T} \boldsymbol{\phi} \boldsymbol{z} \geq\left\|\boldsymbol{\phi}^{-1}\right\|_{2}^{-1}\|\boldsymbol{z}\|^{2} \quad \forall \boldsymbol{z} \in \mathbb{R}^{d}
$$

that is, $\left\|\phi^{-1}\right\|_{2}^{-1}$ is the smallest eigenvalue of $\phi$. For $\chi \in \mathrm{S}_{h, P D}^{1}$, on adopting the notation in the proof of Lemma 5.1] we have that

$$
\chi(\boldsymbol{x})=\sum_{j=0}^{d} \chi\left(P_{j}^{k}\right) \eta_{j}^{k}(\boldsymbol{x}) \quad \forall \boldsymbol{x} \in K_{k}, \quad k=1, \ldots, N_{K} .
$$

Then for $\boldsymbol{v} \in \mathbb{R}^{d}$, with $\|\boldsymbol{v}\|=1$, it follows from (6.27) and (6.26) that

$$
\begin{aligned}
\left\|\chi^{-1}(\boldsymbol{x}) \boldsymbol{v}\right\| & \geq \boldsymbol{v}^{T} \chi^{-1}(\boldsymbol{x}) \boldsymbol{v}=\left(\chi^{-1}(\boldsymbol{x}) \boldsymbol{v}\right)^{T} \boldsymbol{\chi}(\boldsymbol{x})\left(\chi^{-1}(\boldsymbol{x}) \boldsymbol{v}\right) \\
& \geq\left[\sum_{j=0}^{d}\left\|\chi^{-1}\left(P_{j}^{k}\right)\right\|_{2}^{-1} \eta_{j}^{k}(\boldsymbol{x})\right]\left\|\chi^{-1}(\boldsymbol{x}) \boldsymbol{v}\right\|^{2} \quad \forall \boldsymbol{x} \in K_{k} ;
\end{aligned}
$$

where we have noted that $\eta_{j}^{k}(\boldsymbol{x}) \geq 0$, for all $\boldsymbol{x} \in K_{k}$, and $\chi\left(P_{j}^{k}\right) \in \mathbb{R}_{S P D}^{d \times d}, j=$ $0, \ldots, d$. The bound (6.28), on noting (6.25), yields that

$$
\left\|\chi^{-1}(\boldsymbol{x})\right\|_{2} \leq\left[\left[\pi_{h}\left[\left\|\chi^{-1}\right\|_{2}^{-1}\right]\right](\boldsymbol{x})\right]^{-1} \quad \forall \boldsymbol{x} \in K_{k}, \quad k=1, \ldots, N_{K},
$$




$$
\forall \chi \in \mathrm{S}_{h, P D}^{1} .
$$

Hence it follows from (6.25), (6.29) and (5.4) with $\pi_{h}\left[\left\|\chi^{-1}\right\|\right]$ that

$$
\begin{aligned}
\frac{1}{d^{\frac{1}{2}}} \int_{K_{k}}\left\|\chi^{-1}\right\| & \leq \int_{K_{k}}\left[\pi_{h}\left[\left\|\chi^{-1}\right\|_{2}^{-1}\right]\right]^{-1} \leq\left|K_{k}\right|\left\|\pi_{h}\left[\left\|\chi^{-1}\right\|\right]\right\|_{L^{\infty}\left(K_{k}\right)} \\
& \leq C \int_{K_{k}} \pi_{h}\left[\left\|\chi^{-1}\right\|\right] \quad k=1, \ldots, N_{K}, \quad \forall \chi \in \mathrm{S}_{h, P D}^{1},
\end{aligned}
$$

and hence the desired result (6.24).

We note from (1.2b), (1.6) and (2.3) that

$$
\left\|\phi^{-1}\right\| \leq\left\|\left[\beta^{L}(\phi)\right]^{-1}\right\| \quad \forall \phi \in \mathbb{R}_{S P D}^{d \times d} .
$$

Therefore (5.55), (6.16a,b), (5.29), (5.12), (6.31), (6.30) and (5.60a-c) yield that

$$
\begin{aligned}
\sup _{t \in(0, T)} & \left\|\boldsymbol{u}_{\alpha, h}^{L, \Delta t(, \pm)}\right\|_{L^{2}(\mathcal{D})}^{2}+\int_{0}^{T}\left\|\boldsymbol{\nabla} \boldsymbol{u}_{\alpha, h}^{L, \Delta t(, \pm)}\right\|_{L^{2}(\mathcal{D})}^{2} d t \\
& +\int_{0}^{T}\left[\left\|\left[\boldsymbol{\sigma}_{\alpha, h}^{L, \Delta t,+}\right]^{-1}\right\|_{L^{1}(\mathcal{D})}+\frac{\left\|\boldsymbol{u}_{\alpha, h}^{L, \Delta t,+}-\boldsymbol{u}_{\alpha, h}^{L, \Delta t,-}\right\|_{L^{2}(\mathcal{D})}^{2}}{\Delta(t)}\right] d t \leq C, \\
\sup _{t \in(0, T)}\left\|\boldsymbol{\sigma}_{\alpha, h}^{L, \Delta t(, \pm)}\right\|_{L^{2}(\mathcal{D})}^{2} & \\
+ & \int_{0}^{T}\left[\alpha\left\|\boldsymbol{\nabla} \boldsymbol{\sigma}_{\alpha, h}^{L, \Delta t(, \pm)}\right\|_{L^{2}(\mathcal{D})}^{2}+\frac{\left\|\boldsymbol{\sigma}_{\alpha, h}^{L, \Delta t,+}-\boldsymbol{\sigma}_{\alpha, h}^{L, \Delta t,-}\right\|_{L^{2}(\mathcal{D})}^{2}}{\Delta(t)}\right] d t \leq C(L), \\
\int_{0}^{T} & {\left[\left\|\mathcal{S} \frac{\partial \boldsymbol{u}_{\alpha, h}^{L, \Delta t}}{\partial t}\right\|_{H^{1}(\mathcal{D})}^{\frac{4}{\vartheta}}+\left\|\mathcal{E} \frac{\partial \boldsymbol{\sigma}_{\alpha, h}^{L, \Delta t}}{\partial t}\right\|_{H^{1}(\mathcal{D})}^{2}\right] d t \leq C(L, T) ; }
\end{aligned}
$$

where $\vartheta$ is as defined in (6.17).

We are now in a position to prove the following convergence result for $\left(\mathrm{P}_{\alpha, h}^{L, \Delta t}\right)$.

Theorem 6.2. There exists a subsequence of $\left\{\left(\boldsymbol{u}_{\alpha, h}^{L, \Delta t}, \boldsymbol{\sigma}_{\alpha, h}^{L, \Delta t}\right)\right\}_{h>0, \Delta t>0}$, and functions $\left.\boldsymbol{u}_{\alpha}^{L} \in L^{\infty}\left(0, T ;\left[L^{2}(\mathcal{D})\right)\right]^{d}\right) \cap L^{2}(0, T ; \mathrm{V}) \cap W^{1, \frac{4}{\vartheta}}\left(0, T ; \mathrm{V}^{\prime}\right)$ and $\boldsymbol{\sigma}_{\alpha}^{L} \in$ $L^{\infty}\left(0, T ;\left[L^{2}(\mathcal{D})\right]_{S P D}^{d \times d}\right) \cap L^{2}\left(0, T ;\left[H^{1}(\mathcal{D})\right]_{S P D}^{d \times d}\right) \cap H^{1}\left(0, T ;\left(\left[H^{1}(\mathcal{D})\right]_{S}^{d \times d}\right)^{\prime}\right)$ such that, as $h, \Delta t \rightarrow 0_{+}$,

$$
\begin{aligned}
\boldsymbol{u}_{\alpha, h}^{L, \Delta t(, \pm)} \rightarrow \boldsymbol{u}_{\alpha}^{L} & \text { weak }^{*} \text { in } L^{\infty}\left(0, T ;\left[L^{2}(\mathcal{D})\right]^{d}\right), \\
\boldsymbol{u}_{\alpha, h}^{L, \Delta t(, \pm)} \rightarrow \boldsymbol{u}_{\alpha}^{L} & \text { weakly in } L^{2}\left(0, T ;\left[H^{1}(\mathcal{D})\right]^{d}\right), \\
\mathcal{S} \frac{\partial \boldsymbol{u}_{\alpha, h}^{L, \Delta t}}{\partial t} \rightarrow \mathcal{S} \frac{\partial \boldsymbol{u}_{\alpha}^{L}}{\partial t} & \text { weakly in } L^{\frac{4}{\vartheta}}(0, T ; \mathrm{V}), \\
\boldsymbol{u}_{\alpha, h}^{L, \Delta t(, \pm)} \rightarrow \boldsymbol{u}_{\alpha}^{L} & \text { strongly in } L^{2}\left(0, T ;\left[L^{r}(\mathcal{D})\right]^{d}\right),
\end{aligned}
$$

and

$$
\boldsymbol{\sigma}_{\alpha, h}^{L, \Delta t(, \pm)} \rightarrow \boldsymbol{\sigma}_{\alpha}^{L} \quad \text { weak }^{*} \text { in } L^{\infty}\left(0, T ;\left[L^{2}(\mathcal{D})\right]^{d \times d}\right),
$$




$$
\begin{array}{rlrl}
\boldsymbol{\sigma}_{\alpha, h}^{L, \Delta t( \pm)} & \rightarrow \boldsymbol{\sigma}_{\alpha}^{L} & & \text { weakly in } L^{2}\left(0, T ;\left[H^{1}(\mathcal{D})\right]^{d \times d}\right), \\
\mathcal{E} \frac{\partial \boldsymbol{\sigma}_{\alpha, h}^{L, \Delta t}}{\partial t} & \rightarrow \mathcal{E} \frac{\partial \boldsymbol{\sigma}_{\alpha}^{L}}{\partial t} & & \text { weakly in } L^{2}\left(0, T ;\left[H^{1}(\mathcal{D})\right]^{d \times d}\right), \\
\boldsymbol{\sigma}_{\alpha, h}^{L, \Delta t( \pm)} & \rightarrow \boldsymbol{\sigma}_{\alpha}^{L} & & \text { strongly in } L^{2}\left(0, T ;\left[L^{r}(\mathcal{D})\right]^{d \times d}\right), \\
\pi_{h}\left[\beta^{L}\left(\boldsymbol{\sigma}_{\alpha, h}^{L, \Delta t(, \pm)}\right)\right] & \rightarrow \beta^{L}\left(\boldsymbol{\sigma}_{\alpha}^{L}\right) & & \text { strongly in } L^{2}\left(0, T ;\left[L^{2}(\mathcal{D})\right]^{d \times d}\right), \\
\Lambda_{m, p}^{L}\left(\boldsymbol{\sigma}_{\alpha, h}^{L, \Delta t(, \pm)}\right) & \rightarrow \beta^{L}\left(\boldsymbol{\sigma}_{\alpha}^{L}\right) \delta_{m p} & \text { strongly in } L^{2}\left(0, T ;\left[L^{2}(\mathcal{D})\right]^{d \times d}\right), \\
& & m, p=1, \ldots, d,
\end{array}
$$

where $\vartheta$ is defined by (6.17) and $r \in[1, \infty)$ if $d=2$ and $r \in[1,6)$ if $d=3$.

Furthermore, $\left(\boldsymbol{u}_{\alpha}^{L}, \boldsymbol{\sigma}_{\alpha}^{L}\right)$ solve the following problem:

$\left(\boldsymbol{P}_{\alpha}^{L}\right)$ Find $\boldsymbol{u}_{\alpha}^{L} \in L^{\infty}\left(0, T ;\left[L^{2}(\mathcal{D})\right]^{d}\right) \cap L^{2}(0, T ; \mathrm{V}) \cap W^{1, \frac{4}{\vartheta}}\left(0, T ; \mathrm{V}^{\prime}\right)$ and $\boldsymbol{\sigma}_{\alpha}^{L} \in$ $L^{\infty}\left(0, T\left[L^{2}(\mathcal{D})\right]_{S P D}^{d \times d}\right) \cap L^{2}\left(0, T ;\left[H^{1}(\mathcal{D})\right]_{S P D}^{d \times d}\right) \cap H^{1}\left(0, T ;\left(\left[H^{1}(\mathcal{D})\right]_{S}^{d \times d}\right)^{\prime}\right)$ such that

$$
\begin{aligned}
& \int_{0}^{T} \operatorname{Re}\left\langle\frac{\partial \boldsymbol{u}_{\alpha}^{L}}{\partial t}, \boldsymbol{v}\right\rangle_{\mathrm{V}} d t \\
& +\int_{0}^{T} \int_{\mathcal{D}}\left[(1-\varepsilon) \nabla \boldsymbol{u}_{\alpha}^{L}: \nabla \boldsymbol{v}+\operatorname{Re}\left[\left(\boldsymbol{u}_{\alpha}^{L} \cdot \nabla\right) \boldsymbol{u}_{\alpha}^{L}\right] \cdot \boldsymbol{v}\right] d t \\
& =\int_{0}^{T}\langle\boldsymbol{f}, \boldsymbol{v}\rangle_{H_{0}^{1}(\mathcal{D})} d t-\frac{\varepsilon}{\mathrm{Wi}} \int_{0}^{T} \int_{\mathcal{D}} \beta^{L}\left(\boldsymbol{\sigma}_{\alpha}^{L}\right): \nabla \boldsymbol{v} d t \\
& \forall \boldsymbol{v} \in L^{\frac{4}{4-\vartheta}}(0, T ; \mathrm{V}), \\
& \int_{0}^{T}\left\langle\frac{\partial \boldsymbol{\sigma}_{\alpha}^{L}}{\partial t}, \phi\right\rangle_{H^{1}(\mathcal{D})} d t \\
& +\int_{0}^{T} \int_{\mathcal{D}}\left[\left(\boldsymbol{u}_{\alpha}^{L} \cdot \boldsymbol{\nabla}\right)\left[\beta^{L}\left(\boldsymbol{\sigma}_{\alpha}^{L}\right)\right]: \boldsymbol{\phi}+\alpha \boldsymbol{\nabla} \boldsymbol{\sigma}_{\alpha}^{L}:: \boldsymbol{\nabla} \boldsymbol{\phi}\right] d t \\
& =\int_{0}^{T} \int_{\mathcal{D}}\left[2\left(\boldsymbol{\nabla} \boldsymbol{u}_{\alpha}^{L}\right) \beta^{L}\left(\boldsymbol{\sigma}_{\alpha}^{L}\right)-\frac{1}{\mathrm{Wi}}\left(\boldsymbol{\sigma}_{\alpha}^{L}-\boldsymbol{I}\right)\right]: \boldsymbol{\phi} d t \\
& \forall \phi \in L^{2}\left(0, T ;\left[H^{1}(\mathcal{D})\right]_{S}^{d \times d}\right) ; \\
& \forall \boldsymbol{v} \in \mathrm{H}:=\left\{\boldsymbol{w} \in\left[L^{2}(\mathcal{D})\right]^{d}: \operatorname{div} \boldsymbol{w}=0 \text { in } \mathcal{D}\right\}, \\
& \lim _{t \rightarrow 0_{+}} \int_{\mathcal{D}}\left(\boldsymbol{\sigma}_{\alpha}^{L}(t, \boldsymbol{x})-\boldsymbol{\sigma}^{0}(\boldsymbol{x})\right): \chi=0 \quad \forall \chi \in\left[L^{2}(\mathcal{D})\right]_{S P D}^{d \times d} .
\end{aligned}
$$

Proof. The results $(6.33 \mathrm{a}-\mathrm{c})$ follow immediately from the bounds $(6.32 \mathrm{a}) \mathrm{c}$ ) on noting the notation (5.60a-c). The denseness of $\bigcup_{h>0} \mathrm{Q}_{h}^{1}$ in $L^{2}(\mathcal{D})$ and (5.1d) yield that $\boldsymbol{u}_{\alpha}^{L} \in L^{2}(0, T ; \mathrm{V})$. The strong convergence result (6.33d) for $\boldsymbol{u}_{\alpha, h}^{L, \Delta t}$ follows immediately from (6.33a-c) and (6.15) with $\mu_{0}=2, \mu_{1}=4 / \vartheta, \mathcal{Y}_{0}=\left[H^{1}(\mathcal{D})\right]^{d}$, $\mathcal{Y}_{1}=\mathrm{V}^{\prime}$ with norm $\|\mathcal{S} \cdot\|_{H^{1}(\mathcal{D})}$ and $\mathcal{Y}=\left[L^{r}(\mathcal{D})\right]^{d}$ for the stated values of $\vartheta$ and $r$. Here we note that $\mathcal{Y}$ is a Banach space and $\mathcal{Y}_{i}, i=0,1$, are reflexive Banach spaces 
with $\left[L^{r}(\mathcal{D})\right]^{d}$ continuously embedded in $\mathrm{V}^{\prime}$, as $\left[H^{-1}(\mathcal{D})\right]^{d}$ is continuously embedded in $\mathrm{V}^{\prime}$, and $\left[H^{1}(\mathcal{D})\right]^{d}$ compactly embedded in $\left[L^{r}(\mathcal{D})\right]^{d}$ for the stated values of $r$. We now prove (6.33d) for $\boldsymbol{u}_{\alpha, h}^{L, \Delta t, \pm}$. First we obtain from the bound on the last term on the left-hand side of (6.32a) and (5.61) that

$$
\left\|\boldsymbol{u}_{\alpha, h}^{L, \Delta t}-\boldsymbol{u}_{\alpha, h}^{L, \Delta t, \pm}\right\|_{L^{2}\left(0, T, L^{2}(\mathcal{D})\right)}^{2} \leq C \Delta t .
$$

Second, we note from Sobolev embedding that, for all $\eta \in L^{2}\left(0, T ; H^{1}(\mathcal{D})\right)$,

$$
\begin{aligned}
\|\eta\|_{L^{2}\left(0, T ; L^{r}(\mathcal{D})\right)} & \leq\|\eta\|_{L^{2}\left(0, T ; L^{2}(\mathcal{D})\right)}^{\theta}\|\eta\|_{L^{2}\left(0, T ; L^{s}(\mathcal{D})\right)}^{1-\theta} \\
& \leq C\|\eta\|_{L^{2}\left(0, T ; L^{2}(\mathcal{D})\right)}^{\theta}\|\eta\|_{L^{2}\left(0, T ; H^{1}(\mathcal{D})\right)}^{1-\theta}
\end{aligned}
$$

for all $r \in[2, s)$, with any $s \in(2, \infty)$ if $d=2$ or any $s \in(2,6]$ if $d=3$, and $\theta=[2(s-r)] /[r(s-2)] \in(0,1]$. Hence, combining (6.36), (6.37) and (6.33d) for $\boldsymbol{u}_{\alpha, h}^{L, \Delta t}$ yields (6.33d) for $\boldsymbol{u}_{\alpha, h}^{L, \Delta t, \pm}$.

Similarly, the results (6.34a $\mathrm{c})$ follow immediately from $(6.32 \mathrm{~b}, \mathrm{c})$. The strong convergence result (6.34d) for $\sigma_{\alpha, h}^{L, \Delta t}$ follows immediately from (6.34a-c), (6.11) and (6.15) with $\mu_{0}=\mu_{1}=2, \mathcal{Y}_{0}=\left[H^{1}(\mathcal{D})\right]^{d \times d}, \mathcal{Y}_{1}=\left[H^{-1}(\mathcal{D})\right]^{d \times d}$ and $\mathcal{Y}=\left[L^{r}(\mathcal{D})\right]^{d \times d}$ for the stated values $r$. Similarly to (6.36), the second bound in (6.32b) then yields that (6.34d) holds for $\sigma_{\alpha, h}^{L, \Delta t(, \pm)}$.

Since $\boldsymbol{\sigma}_{\alpha, h}^{L, \Delta t(, \pm)} \in L^{2}\left(0, T ; \mathrm{S}_{h, P D}^{1}\right)$, it follows that $\boldsymbol{\sigma}_{\alpha}^{L}$ is symmetric non-negative definite a.e. in $\mathcal{D}_{T}$. We now establish that $\sigma_{\alpha}^{L}$ is symmetric positive definite a.e. in $\mathcal{D}_{T}$. Assume that $\boldsymbol{\sigma}_{\alpha}^{L}$ is not symmetric positive definite a.e. in $\mathcal{D}_{T}^{0} \subset \mathcal{D}_{T}$. Let $\boldsymbol{v} \in L^{2}\left(0, T ;\left[L^{2}(\mathcal{D})\right]^{d}\right)$ be such that $\boldsymbol{\sigma}_{\alpha}^{L} \boldsymbol{v}=\mathbf{0}$ with $\|\boldsymbol{v}\|=1$ a.e. in $\mathcal{D}_{T}^{0}$ and $\boldsymbol{v}=\mathbf{0}$ a.e. in $\mathcal{D} \backslash \mathcal{D}_{T}^{0}$. We then have from (6.32a that

$$
\begin{aligned}
\left|\mathcal{D}_{T}^{0}\right| & =\int_{0}^{T} \int_{\mathcal{D}}\|\boldsymbol{v}\|^{2} d t=\int_{0}^{T} \int_{\mathcal{D}}\left(\left[\boldsymbol{\sigma}_{\alpha, h}^{L, \Delta t,+}\right]^{-\frac{1}{2}} \boldsymbol{v}\right):\left(\left[\boldsymbol{\sigma}_{\alpha, h}^{L, \Delta t,+}\right]^{\frac{1}{2}} \boldsymbol{v}\right) d t \\
& \leq C\left(\int_{0}^{T} \int_{\mathcal{D}} \boldsymbol{\sigma}_{\alpha, h}^{L, \Delta t,+}::\left(\boldsymbol{v} \boldsymbol{v}^{T}\right) d t\right)^{\frac{1}{2}} .
\end{aligned}
$$

Hence it follows from (6.38) and (6.34d) that $\left|\mathcal{D}_{T}^{0}\right|=0$.

Finally, the desired results (6.34e,f) follow immediately from (5.64) the second bound in (6.32b), (2.17), (6.34d) and the fact that $\sigma_{\alpha}^{L} \in L^{\infty}\left(0, T ;\left[L^{2}(\mathcal{D})\right]_{S P D}^{d \times d}\right)$.

It remains to prove that $\left(\boldsymbol{u}_{\alpha}^{L}, \boldsymbol{\sigma}_{\alpha}^{L}\right)$ solves $\left(\mathrm{P}_{\alpha}^{L}\right)$. It follows from (5.3), (6.32a $\mathrm{c}$ ), (6.33a-d), (6.34e), (3.10b), (6.12) and (3.13) that we may pass to the limit, $h, \Delta t \rightarrow$ $0_{+}$, in the $L$-dependent version of (5.62) to obtain that $\left(\boldsymbol{u}_{\alpha}^{L}, \boldsymbol{\sigma}_{\alpha}^{L}\right)$ satisfy (6.35a). It also follows from (5.28a), (5.3), (6.33c,d) and as $\mathrm{V}$ is dense in $\mathrm{H}$ that $\boldsymbol{u}_{\alpha}^{L}(0, \cdot)=\boldsymbol{u}^{0}(\cdot)$ in the required sense; see (6.35c) and Lemma 1.4 on p179 in Temam. Tem84

It follows from (6.34a-f), (6.33b d), (6.10), (6.32a-c), (6.1a b), (1.4a) and as $\boldsymbol{u}_{\alpha}^{L} \in L^{2}(0, T ; \mathrm{V})$ that we may pass to the limit $h, \Delta t \rightarrow 0_{+}$in the $L$-dependent version of (5.63) with $\chi=\pi_{h} \phi$ to obtain (6.35b) for any $\phi \in C_{0}^{\infty}\left(0, T ;\left[C^{\infty}(\overline{\mathcal{D}})\right]_{S}^{d \times d}\right)$. For example, in order to pass to the limit on the first term in the $L$-dependent 
version of (5.63), we note that

$$
\begin{aligned}
& \int_{0}^{T} \int_{\mathcal{D}} \pi_{h} {\left[\left(\frac{\partial \boldsymbol{\sigma}_{\alpha, h}^{L, \Delta t}}{\partial t}+\frac{1}{\mathrm{Wi}} \boldsymbol{\sigma}_{\alpha, h}^{L, \Delta t,+}\right): \pi_{h} \phi\right] d t } \\
&=\int_{0}^{T} \int_{\mathcal{D}}\left\{\left(\frac{\partial \boldsymbol{\sigma}_{\alpha, h}^{L, \Delta t}}{\partial t}+\frac{1}{\mathrm{Wi}} \boldsymbol{\sigma}_{\alpha, h}^{L, \Delta t,+}\right): \pi_{h} \phi+\left(I-\pi_{h}\right)\left[\boldsymbol{\sigma}_{\alpha, h}^{L, \Delta t}: \pi_{h}\left[\frac{\partial \phi}{\partial t}\right]\right]\right\} d t \\
& \quad-\frac{1}{\mathrm{Wi}} \int_{0}^{T} \int_{\mathcal{D}}\left(I-\pi_{h}\right)\left[\boldsymbol{\sigma}_{\alpha, h}^{L, \Delta t,+}: \pi_{h} \phi\right] d t
\end{aligned}
$$

The desired result (6.35b) then follows from noting that $C_{0}^{\infty}\left(0, T ;\left[C^{\infty}(\overline{\mathcal{D}})\right]_{S}^{d \times d}\right)$ is dense in $L^{2}\left(0, T ;\left[H^{1}(\mathcal{D})\right]_{S}^{d \times d}\right)$. Finally, it follows from (5.28b), 66.34c, d), 66.1a , b) and (5.12) that $\sigma_{\alpha}^{L}(0, \cdot)=\sigma^{0}(\cdot)$ in the required sense; see (6.35c) and Lemma 1.4 on p179 in Temam. Tem84

Remark 6.1. It follows from (6.32a, b), 66.33a, b) and (6.34a, b) that

$$
\begin{gathered}
\sup _{t \in(0, T)}\left\|\boldsymbol{u}_{\alpha}^{L}\right\|_{L^{2}(\mathcal{D})}^{2}+\int_{0}^{T}\left\|\nabla \boldsymbol{u}_{\alpha}^{L}\right\|_{L^{2}(\mathcal{D})}^{2} d t \leq C, \\
\sup _{t \in(0, T)}\left\|\boldsymbol{\sigma}_{\alpha}^{L}\right\|_{L^{2}(\mathcal{D})}^{2}+\alpha \int_{0}^{T}\left\|\nabla \boldsymbol{\sigma}_{\alpha}^{L}\right\|_{L^{2}(\mathcal{D})}^{2} d t \leq C(L) .
\end{gathered}
$$

Hence, although we have introduced a cut-off $L \gg 1$ to certain terms, and added diffusion with a positive coefficient $\alpha$ in the stress equation compared to the standard Oldroyd-B model; the bound (6.40a) on the velocity $\boldsymbol{u}_{\alpha}^{L}$ is independent of the parameters $L$ and $\alpha$, where $\left(\boldsymbol{u}_{\alpha}^{L}, \boldsymbol{\sigma}_{\alpha}^{L}\right)$ solves $\left(\mathrm{P}_{\alpha}^{L}\right)$, (6.35a-c).

\section{Convergence of $\left(\mathrm{P}_{\alpha, h}^{\Delta t}\right)$ to $\left(\mathrm{P}_{\alpha}\right)$ in the case $d=2$}

First, we recall the discrete Gronwall inequality:

$$
\begin{array}{rlrl}
\left(r^{0}\right)^{2}+\left(s^{0}\right)^{2} & \leq\left(q^{0}\right)^{2}, \\
\left(r^{m}\right)^{2}+\left(s^{m}\right)^{2} & \leq \sum_{n=0}^{m-1}\left(\eta^{n}\right)^{2}\left(r^{n}\right)^{2}+\sum_{n=0}^{m}\left(q^{n}\right)^{2} \quad & & m \geq 1 \\
\Rightarrow \quad\left(r^{m}\right)^{2}+\left(s^{m}\right)^{2} & \leq \exp \left(\sum_{n=0}^{m-1}\left(\eta^{n}\right)^{2}\right) \sum_{n=0}^{m}\left(q^{n}\right)^{2} & & m \geq 1 .
\end{array}
$$

Theorem 7.1. Under the assumptions of Theorem 5.2, there exists a solution $\left\{\left(\boldsymbol{u}_{\alpha, h}^{n}, \boldsymbol{\sigma}_{\alpha, h}^{n}\right)\right\}_{n=1}^{N_{T}} \in\left[\mathrm{V}_{h}^{1} \times \mathrm{S}_{h, P D}^{1}\right]^{N_{T}}$ of $\left(P_{\alpha, h}^{\Delta t}\right)$ such that the bounds 5.54) and (5.55) hold.

If $d=2, \alpha \leq \frac{1}{2 W i}$ and $\Delta t \leq C_{\star}\left(\zeta^{-1}\right) \alpha^{1+\zeta} h^{2}$, for $a \zeta>0$, then the following bounds hold:

$$
\max _{n=0, \ldots, N_{T}} \int_{\mathcal{D}} \pi_{h}\left[\left\|\boldsymbol{\sigma}_{\alpha, h}^{n}\right\|^{2}\right]+\sum_{n=1}^{N_{T}} \int_{\mathcal{D}}\left[\Delta t_{n} \alpha\left\|\nabla \boldsymbol{\sigma}_{\alpha, h}^{n}\right\|^{2}+\pi_{h}\left[\left\|\boldsymbol{\sigma}_{\alpha, h}^{n}-\boldsymbol{\sigma}_{\alpha, h}^{n-1}\right\|^{2}\right]\right]
$$




$$
+\sum_{n=1}^{N_{T}} \Delta t_{n}\left\|\mathcal{S}\left(\frac{\boldsymbol{u}_{\alpha, h}^{n}-\boldsymbol{u}_{\alpha, h}^{n-1}}{\Delta t_{n}}\right)\right\|_{H^{1}(\mathcal{D})}^{\frac{4}{\vartheta}} \leq C\left(\alpha^{-1}, T\right) ;
$$

where $\vartheta \in(2,4)$.

Proof. Existence and the bounds (5.54) and (5.55) were proved in Theorem 5.2.

On choosing $\phi \equiv \sigma_{\alpha, h}^{n}$ in the $L$-independent version of (5.50b), it follows from (3.14) and on applying a Youngs' inequality for any $\zeta>0$ that

$$
\begin{aligned}
\frac{1}{2} \int_{\mathcal{D}} & \pi_{h}\left[\left\|\boldsymbol{\sigma}_{\alpha, h}^{n}\right\|^{2}+\left\|\boldsymbol{\sigma}_{\alpha, h}^{n}-\boldsymbol{\sigma}_{\alpha, h}^{n-1}\right\|^{2}\right]+\Delta t_{n} \alpha \int_{\mathcal{D}}\left\|\boldsymbol{\nabla} \boldsymbol{\sigma}_{\alpha, h}^{n}\right\|^{2}+\frac{\Delta t_{n}}{2 \mathrm{Wi}} \int_{\mathcal{D}} \pi_{h}\left[\left\|\boldsymbol{\sigma}_{\alpha, h}^{n}\right\|^{2}\right] \\
\leq & \frac{1}{2} \int_{\mathcal{D}} \pi_{h}\left[\left\|\boldsymbol{\sigma}_{\alpha, h}^{n-1}\right\|^{2}\right]+\frac{\Delta t_{n} d|\mathcal{D}|}{2 \mathrm{Wi}}+2 \Delta t_{n} \int_{\mathcal{D}} \boldsymbol{\nabla} \boldsymbol{u}_{\alpha, h}^{n}: \pi_{h}\left[\left(\boldsymbol{\sigma}_{\alpha, h}^{n}\right)^{2}\right] \\
& \quad+\Delta t_{n} \int_{\mathcal{D}} \sum_{m=1}^{d} \sum_{p=1}^{d}\left[\boldsymbol{u}_{\alpha, h}^{n-1}\right]_{m} \Lambda_{m, p}\left(\boldsymbol{\sigma}_{\alpha, h}^{n}\right): \frac{\partial \boldsymbol{\sigma}_{\alpha, h}^{n}}{\partial \boldsymbol{x}_{p}} \\
\leq & \frac{1}{2} \int_{\mathcal{D}} \pi_{h}\left[\left\|\boldsymbol{\sigma}_{\alpha, h}^{n-1}\right\|^{2}\right]+C \Delta t_{n}\left[1+\left\|\boldsymbol{\nabla} \boldsymbol{u}_{\alpha, h}^{n}\right\|_{L^{2}(\mathcal{D})}\left\|\pi_{h}\left[\left(\boldsymbol{\sigma}_{\alpha, h}^{n}\right)^{2}\right]\right\|_{L^{2}(\mathcal{D})}\right] \\
& +C \Delta t_{n}\left\|\boldsymbol{u}_{\alpha, h}^{n-1}\right\|_{L_{\frac{2(2+\zeta)}{\zeta}}(\mathcal{D})}\left\|\Lambda_{m, p}\left(\boldsymbol{\sigma}_{\alpha, h}^{n}\right)\right\|_{L^{2+\zeta(\mathcal{D})}}\left\|\boldsymbol{\nabla} \boldsymbol{\sigma}_{\alpha, h}^{n}\right\|_{L^{2}(\mathcal{D})} .
\end{aligned}
$$

It follows from (5.12), (1.4b), (5.4) and (6.14), as $d=2$, that

$$
\begin{aligned}
\left\|\pi_{h}\left[\left(\boldsymbol{\sigma}_{\alpha, h}^{n}\right)^{2}\right]\right\|_{L^{2}(\mathcal{D})}^{2} & =\int_{\mathcal{D}}\left\|\pi_{h}\left[\left(\boldsymbol{\sigma}_{\alpha, h}^{n}\right)^{2}\right]\right\|^{2} \leq \int_{\mathcal{D}} \pi_{h}\left[\left\|\left(\boldsymbol{\sigma}_{\alpha, h}^{n}\right)^{2}\right\|^{2}\right] \leq \int_{\mathcal{D}} \pi_{h}\left[\left\|\boldsymbol{\sigma}_{\alpha, h}^{n}\right\|^{4}\right] \\
& =\sum_{k=1}^{N_{K}} \int_{K_{k}} \pi_{h}\left[\left\|\boldsymbol{\sigma}_{\alpha, h}^{n}\right\|^{4}\right] \leq \sum_{k=1}^{N_{K}}\left|K_{k}\right|\left\|\boldsymbol{\sigma}_{\alpha, h}^{n}\right\|_{L^{\infty}\left(K_{k}\right)}^{4} \\
& \leq C \sum_{k=1}^{N_{K}}\left|K_{k}\right|\left(\left|K_{k}\right|^{-1}\left\|\boldsymbol{\sigma}_{\alpha, h}^{n}\right\|_{L^{1}\left(K_{k}\right)}\right)^{4} \leq C \sum_{k=1}^{N_{K}}\left\|\boldsymbol{\sigma}_{\alpha, h}^{n}\right\|_{L^{4}\left(K_{k}\right)}^{4} \\
& =C\left\|\boldsymbol{\sigma}_{\alpha, h}^{n}\right\|_{L^{4}(\mathcal{D})}^{4} \leq C\left\|\boldsymbol{\sigma}_{\alpha, h}^{n}\right\|_{L^{2}(\mathcal{D})}^{2}\left\|\boldsymbol{\sigma}_{\alpha, h}^{n}\right\|_{H^{1}(\mathcal{D})}^{2} .
\end{aligned}
$$

Similarly, it follows from the $\delta$-independent versions of (5.22), (5.21a, b), recall Remark 5.1, (5.25), (5.4) and (6.14) that for all $\zeta>0$

$$
\begin{aligned}
\left\|\Lambda_{m, p}\left(\boldsymbol{\sigma}_{\alpha, h}^{n}\right)\right\|_{L^{2+\zeta(\mathcal{D})}}^{2+\zeta} & \leq \sum_{k=1}^{N_{K}}\left|K_{k}\right|\left\|\Lambda_{m, p}\left(\boldsymbol{\sigma}_{\alpha, h}^{n}\right)\right\|_{L^{\infty}\left(K_{k}\right)}^{2+\zeta} \leq C \sum_{k=1}^{N_{K}}\left|K_{k}\right|\left\|\boldsymbol{\sigma}_{\alpha, h}^{n}\right\|_{L^{\infty}\left(K_{k}\right)}^{2+\zeta} \\
& =C\left\|\boldsymbol{\sigma}_{\alpha, h}^{n}\right\|_{L^{2+\zeta(\mathcal{D})}}^{2+\zeta} \leq C(\zeta)\left\|\boldsymbol{\sigma}_{\alpha, h}^{n}\right\|_{L^{2}(\mathcal{D})}^{2}\left\|\boldsymbol{\sigma}_{\alpha, h}^{n}\right\|_{H^{1}(\mathcal{D})}^{\zeta} . \quad(7.5)
\end{aligned}
$$

In addition, we note from (6.14), (1.8) and (5.55) that for all $\zeta>0$

$$
\left\|\boldsymbol{u}_{\alpha, h}^{n-1}\right\|_{L^{\frac{2(2+\zeta)}{\zeta}(\mathcal{D})}} \leq C\left(\zeta^{-1}\right)\left\|\boldsymbol{u}_{\alpha, h}^{n-1}\right\|_{L^{2}(\mathcal{D})}^{\frac{\zeta}{2+\zeta}}\left\|\boldsymbol{u}_{\alpha, h}^{n-1}\right\|_{H^{1}(\mathcal{D})}^{\frac{2}{2+\zeta}} \leq C\left(\zeta^{-1}\right)\left\|\nabla \boldsymbol{u}_{\alpha, h}^{n-1}\right\|_{L^{2}(\mathcal{D})}^{\frac{2}{2+\zeta}} .
$$


Combining (7.3), (7.4), (7.5) and (7.6), and on noting (5.12) and that $\alpha \leq \frac{1}{2 \mathrm{Wi}}$, yields on applying a Young's inequality that for all $\zeta>0$

$$
\begin{aligned}
& \int_{\mathcal{D}} \pi_{h}\left[\left\|\boldsymbol{\sigma}_{\alpha, h}^{n}\right\|^{2}+\left\|\boldsymbol{\sigma}_{\alpha, h}^{n}-\boldsymbol{\sigma}_{\alpha, h}^{n-1}\right\|^{2}\right]+\Delta t_{n} \alpha \int_{\mathcal{D}}\left\|\boldsymbol{\nabla} \boldsymbol{\sigma}_{\alpha, h}^{n}\right\|^{2}+\frac{\Delta t_{n}}{2 \mathrm{Wi}} \int_{\mathcal{D}} \pi_{h}\left[\left\|\boldsymbol{\sigma}_{\alpha, h}^{n}\right\|^{2}\right] \\
& \leq \int_{\mathcal{D}} \pi_{h}\left[\left\|\boldsymbol{\sigma}_{\alpha, h}^{n-1}\right\|^{2}\right]+C \Delta t_{n} \\
& \quad+C\left(\zeta^{-1}\right) \Delta t_{n} \alpha^{-(1+\zeta)}\left[\left\|\nabla \boldsymbol{u}_{\alpha, h}^{n}\right\|_{L^{2}(\mathcal{D})}^{2}+\left\|\boldsymbol{\nabla} \boldsymbol{u}_{\alpha, h}^{n-1}\right\|_{L^{2}(\mathcal{D})}^{2}\right] \int_{\mathcal{D}} \pi_{h}\left[\left\|\boldsymbol{\sigma}_{\alpha, h}^{n}\right\|^{2}\right] .
\end{aligned}
$$

Hence, summing (7.7) from $n=1, \ldots, m$ for $m=1, \ldots, N_{T}$ yields, for any $\zeta>0$ that

$$
\begin{aligned}
\int_{\mathcal{D}} \pi_{h}[ & \left.\left\|\boldsymbol{\sigma}_{\alpha, h}^{m}\right\|^{2}\right]+\sum_{n=1}^{m} \Delta t_{n} \int_{\mathcal{D}}\left[\alpha\left\|\nabla \boldsymbol{\sigma}_{\alpha, h}^{n}\right\|^{2}+\frac{1}{2 \mathrm{Wi}} \pi_{h}\left[\left\|\boldsymbol{\sigma}_{\alpha, h}^{n}\right\|^{2}\right]\right] \\
+ & \sum_{n=1}^{m} \int_{\mathcal{D}} \pi_{h}\left[\left\|\boldsymbol{\sigma}_{\alpha, h}^{n}-\boldsymbol{\sigma}_{\alpha, h}^{n-1}\right\|^{2}\right] \\
\leq & \int_{\mathcal{D}} \pi_{h}\left[\left\|\boldsymbol{\sigma}_{\alpha, h}^{0}\right\|^{2}\right]+C \\
& +C\left(\zeta^{-1}\right) \alpha^{-(1+\zeta)} \sum_{n=1}^{m} \Delta t_{n}\left[\sum_{k=n-1}^{n}\left\|\nabla \boldsymbol{u}_{\alpha, h}^{k}\right\|_{L^{2}(\mathcal{D})}^{2}\right] \int_{\mathcal{D}} \pi_{h}\left[\left\|\boldsymbol{\sigma}_{\alpha, h}^{n}\right\|^{2}\right]
\end{aligned}
$$

Applying the discrete Gronwall inequality (7.1) to (7.8), and noting (5.27), (5.13), (5.29), (5.55), (5.5) and that $\Delta t \leq C_{\star}\left(\zeta^{-1}\right) \alpha^{1+\zeta} h^{2}$, for a $\zeta>0$ where $C_{\star}\left(\zeta^{-1}\right)$ is sufficiently small, yields the first three bounds in (7.2).

Similarly to (6.19), on choosing $\boldsymbol{w}=\mathcal{R}_{h}\left[\mathcal{S}\left(\frac{\boldsymbol{u}_{\alpha, h}^{n}-\boldsymbol{u}_{\alpha, h}^{n-1}}{\Delta t_{n}}\right)\right] \in \mathrm{V}_{h}^{1}$ in the $L$ independent version of (5.50a) yields, on noting (6.4), (6.13), (6.8) and Sobolev embedding, that

$$
\begin{gathered}
\operatorname{Re}\left\|\mathcal{S}\left(\frac{\boldsymbol{u}_{\alpha, h}^{n}-\boldsymbol{u}_{\alpha, h}^{n-1}}{\Delta t_{n}}\right)\right\|_{H^{1}(\mathcal{D})}^{2}=\operatorname{Re} \int_{\mathcal{D}} \frac{\boldsymbol{u}_{\alpha, h}^{n}-\boldsymbol{u}_{\alpha, h}^{n-1}}{\Delta t_{n}} \cdot \mathcal{R}_{h}\left[\mathcal{S}\left(\frac{\boldsymbol{u}_{\alpha, h}^{n}-\boldsymbol{u}_{\alpha, h}^{n-1}}{\Delta t_{n}}\right)\right] \\
\leq C\left[\left\|\boldsymbol{\sigma}_{\alpha, h}^{n}\right\|_{L^{2}(\mathcal{D})}^{2}+\left\|\boldsymbol{\nabla} \boldsymbol{u}_{\alpha, h}^{n}\right\|_{L^{2}(\mathcal{D})}^{2}+\|\| \boldsymbol{u}_{\alpha, h}^{n-1}\|\| \boldsymbol{u}_{\alpha, h}^{n}\|\|_{L^{2}(\mathcal{D})}^{2}\right. \\
\left.\quad+\|\| \boldsymbol{u}_{\alpha, h}^{n-1}\|\| \boldsymbol{\nabla} \boldsymbol{u}_{\alpha, h}^{n}\|\|_{L^{1+\theta}(\mathcal{D})}^{2}+\left\|\boldsymbol{f}^{n}\right\|_{H^{-1}(\mathcal{D})}^{2}\right]
\end{gathered}
$$

for any $\theta>0$ as $d=2$. On taking the $\frac{2}{\vartheta}$ power of both sides of (7.9), multiplying by $\Delta t_{n}$, summing from $n=1, \ldots, N_{T}$ and noting the $L$-independent versions of (6.20) and (6.21a) with $\theta=(\vartheta-2) /(6-\vartheta)$, (5.27), (3.10a), (5.55), (5.29), (5.12) and the first bound in (7.2) yields the last bound in (7.2).

It follows from (5.55), (7.2), (5.29), (5.12), (6.30) and (5.60a-c) that

$$
\sup _{t \in(0, T)}\left\|\boldsymbol{u}_{\alpha, h}^{\Delta t(, \pm)}\right\|_{L^{2}(\mathcal{D})}^{2}+\int_{0}^{T}\left\|\nabla \boldsymbol{u}_{\alpha, h}^{\Delta t(, \pm)}\right\|_{L^{2}(\mathcal{D})}^{2} d t
$$




$$
+\int_{0}^{T}\left[\left\|\left[\boldsymbol{\sigma}_{\alpha, h}^{\Delta t,+}\right]^{-1}\right\|_{L^{1}(\mathcal{D})}+\frac{\left\|\boldsymbol{u}_{\alpha, h}^{\Delta t,+}-\boldsymbol{u}_{\alpha, h}^{\Delta t,-}\right\|_{L^{2}(\mathcal{D})}^{2}}{\Delta(t)}\right] d t \leq C
$$

and

$$
\begin{aligned}
\sup _{t \in(0, T)}\left\|\boldsymbol{\sigma}_{\alpha, h}^{\Delta t(, \pm)}\right\|_{L^{2}(\mathcal{D})}^{2}+\int_{0}^{T}\left[\alpha\left\|\nabla \boldsymbol{\sigma}_{\alpha, h}^{\Delta t(, \pm)}\right\|_{L^{2}(\mathcal{D})}^{2}+\frac{\left\|\boldsymbol{\sigma}_{\alpha, h}^{\Delta t,+}-\boldsymbol{\sigma}_{\alpha, h}^{\Delta t,-}\right\|_{L^{2}(\mathcal{D})}^{2}}{\Delta(t)}\right] d t \\
+\int_{0}^{T}\left\|\mathcal{S} \frac{\partial \boldsymbol{u}_{\alpha, h}^{\Delta t}}{\partial t}\right\|_{H^{1}(\mathcal{D})}^{\frac{4}{\vartheta}} d t \leq C\left(\alpha^{-1}, T\right),
\end{aligned}
$$

where $\vartheta \in(2,4)$.

We note that we have no control on the time derivative of $\sigma_{\alpha, h}^{\Delta t}$ in (7.10b). This is because if we choose $\phi=\mathcal{P}_{h}\left[\mathcal{E}\left(\frac{\sigma_{\alpha, h}^{n}-\boldsymbol{\sigma}_{\alpha, h}^{n-1}}{\Delta t_{n}}\right)\right] \in \mathrm{S}_{h}^{1}$ in the $L$-independent version of (5.50b), the terms involving $\boldsymbol{u}_{\alpha, h}^{m}, m=n-1$ and $m=n$, cannot now be controlled in the absence of the cut-off on $\boldsymbol{\sigma}_{\alpha, h}^{n}$. We are now in a position to prove the following convergence result for $\left(\mathrm{P}_{\alpha, h}^{\Delta t}\right)$. The key difference between the following theorem and Theorem 6.2 for $\left(\mathrm{P}_{\alpha, h}^{L, \Delta t}\right)$ is that no control on the time derivative of $\boldsymbol{\sigma}_{\alpha, h}^{\Delta t}$ in (7.10b) implies no strong convergence for $\boldsymbol{\sigma}_{\alpha, h}^{\Delta t(, \pm)}$.

Theorem 7.2. Let all the assumptions of Theorem 7.1 hold. Then there exists a subsequence of $\left\{\left(\boldsymbol{u}_{\alpha, h}^{\Delta t}, \boldsymbol{\sigma}_{\alpha, h}^{\Delta t}\right)\right\}_{h>0, \Delta t>0}$, and functions $\left.\boldsymbol{u}_{\alpha} \in L^{\infty}\left(0, T ;\left[L^{2}(\mathcal{D})\right)\right]^{2}\right) \cap$ $L^{2}(0, T ; \mathrm{V}) \cap W^{1, \frac{4}{\vartheta}}\left(0, T ; \mathrm{V}^{\prime}\right)$ and $\boldsymbol{\sigma}_{\alpha} \in L^{\infty}\left(0, T ;\left[L^{2}(\mathcal{D})\right]_{S P D}^{2 \times 2}\right) \cap L^{2}\left(0, T ;\left[H^{1}(\mathcal{D})\right]_{S P D}^{2 \times 2}\right)$ such that, as $h, \Delta t \rightarrow 0_{+}$,

$$
\begin{aligned}
\boldsymbol{u}_{\alpha, h}^{\Delta t(, \pm)} \rightarrow \boldsymbol{u}_{\alpha} & \text { weak }^{*} \text { in } L^{\infty}\left(0, T ;\left[L^{2}(\mathcal{D})\right]^{2}\right), \\
\boldsymbol{u}_{\alpha, h}^{\Delta t(, \pm)} \rightarrow \boldsymbol{u}_{\alpha} & \text { weakly in } L^{2}\left(0, T ;\left[H^{1}(\mathcal{D})\right]^{2}\right), \\
\mathcal{S} \frac{\partial \boldsymbol{u}_{\alpha, h}^{\Delta t}}{\partial t} \rightarrow \mathcal{S} \frac{\partial \boldsymbol{u}_{\alpha}}{\partial t} & \text { weakly in } L^{\frac{4}{\vartheta}}(0, T ; \mathrm{V}), \\
\boldsymbol{u}_{\alpha, h}^{\Delta t(, \pm)} \rightarrow \boldsymbol{u}_{\alpha} & \text { strongly in } L^{2}\left(0, T ;\left[L^{r}(\mathcal{D})\right]^{2}\right),
\end{aligned}
$$

and

$$
\begin{array}{rlrl}
\boldsymbol{\sigma}_{\alpha, h}^{\Delta t(, \pm)} & \rightarrow \boldsymbol{\sigma}_{\alpha} & & \text { weak }^{*} \text { in } L^{\infty}\left(0, T ;\left[L^{2}(\mathcal{D})\right]^{2 \times 2}\right), \\
\boldsymbol{\sigma}_{\alpha, h}^{\Delta t(, \pm)} & \rightarrow \boldsymbol{\sigma}_{\alpha} & & \text { weakly in } L^{2}\left(0, T ;\left[H^{1}(\mathcal{D})\right]^{2 \times 2}\right), \\
\Lambda_{m, p}\left(\boldsymbol{\sigma}_{\alpha, h}^{\Delta t(, \pm)}\right) & \rightarrow \boldsymbol{\sigma}_{\alpha} \delta_{m p} & & \text { weakly in } L^{2}\left(0, T ;\left[L^{2}(\mathcal{D})\right]^{2 \times 2}\right), \\
& & m, p=1,2,
\end{array}
$$

where $\vartheta \in(2,4)$ and $r \in[1, \infty)$.

Furthermore, $\left(\boldsymbol{u}_{\alpha}, \boldsymbol{\sigma}_{\alpha}\right)$ solve the following problem: 
$\left(\boldsymbol{P}_{\alpha}\right)$ Find $\boldsymbol{u}_{\alpha} \in L^{\infty}\left(0, T ;\left[L^{2}(\mathcal{D})\right]^{2}\right) \cap L^{2}(0, T ; \mathrm{V}) \cap W^{1, \frac{4}{\vartheta}}\left(0, T ; \mathrm{V}^{\prime}\right)$ and $\boldsymbol{\sigma}_{\alpha} \in$ $L^{\infty}\left(0, T ;\left[L^{2}(\mathcal{D})\right]_{S P D}^{2 \times 2}\right) \cap L^{2}\left(0, T ;\left[H^{1}(\mathcal{D})\right]_{S P D}^{2 \times 2}\right)$ such that

$$
\begin{gathered}
\int_{0}^{T} \operatorname{Re}\left\langle\frac{\partial \boldsymbol{u}_{\alpha}}{\partial t}, \boldsymbol{v}\right\rangle_{\mathrm{V}} d t \\
+\int_{0}^{T} \int_{\mathcal{D}}\left[(1-\varepsilon) \boldsymbol{\nabla} \boldsymbol{u}_{\alpha}: \nabla \boldsymbol{v}+\operatorname{Re}\left[\left(\boldsymbol{u}_{\alpha} \cdot \boldsymbol{\nabla}\right) \boldsymbol{u}_{\alpha}\right] \cdot \boldsymbol{v}\right] d t \\
\quad=\int_{0}^{T}\langle\boldsymbol{f}, \boldsymbol{v}\rangle_{H_{0}^{1}(\mathcal{D})} d t-\frac{\varepsilon}{\mathrm{Wi}} \int_{0}^{T} \int_{\mathcal{D}} \boldsymbol{\sigma}_{\alpha}: \boldsymbol{\nabla} \boldsymbol{v} d t \quad \forall \boldsymbol{v} \in L^{\frac{4}{4-\vartheta}}(0, T ; \mathrm{V}), \\
-\int_{0}^{T} \int_{\mathcal{D}} \boldsymbol{\sigma}_{\alpha}: \frac{\partial \phi}{\partial t} d t-\int_{\mathcal{D}} \boldsymbol{\sigma}^{0}: \boldsymbol{\phi} \\
\quad+\int_{0}^{T} \int_{\mathcal{D}}\left[\left(\boldsymbol{u}_{\alpha} \cdot \boldsymbol{\nabla}\right) \boldsymbol{\sigma}_{\alpha}: \boldsymbol{\phi}+\alpha \boldsymbol{\nabla} \boldsymbol{\sigma}_{\alpha}:: \nabla \phi\right] d t \\
\quad=\int_{0}^{T} \int_{\mathcal{D}}\left[2\left(\boldsymbol{\nabla} \boldsymbol{u}_{\alpha}\right) \boldsymbol{\sigma}_{\alpha}-\frac{1}{\mathrm{Wi}}\left(\boldsymbol{\sigma}_{\alpha}-\boldsymbol{I}\right)\right]: \boldsymbol{\phi} d t \\
\quad \forall \boldsymbol{\phi} \in L^{2}\left(0, T ;\left[H^{1}(\mathcal{D})\right]_{S}^{2 \times 2}\right) \cap W_{0}^{1,1}\left(-T, T ;\left[L^{2}(\mathcal{D})\right]_{S}^{2 \times 2}\right) ; \quad \text { (7.13b) } \\
\lim _{t \rightarrow 0_{+}} \int_{\mathcal{D}}\left(\boldsymbol{u}_{\alpha}(t, \boldsymbol{x})-\boldsymbol{u}^{0}(\boldsymbol{x})\right) \cdot \boldsymbol{v}=0 \quad \forall \boldsymbol{v} \in \mathrm{H} .
\end{gathered}
$$

Proof. The results (7.11a d) and (7.12a b) follow immediately from the bounds (7.10a b), as in the proof of Theorem 6.2 Similarly, the proof of positive definiteness of $\boldsymbol{\sigma}_{\alpha}$ follows as in Theorem 6.2 that is, (6.38) and the weak convergence (7.12a) is adequate for this. The result (7.12C) follows from (7.12a), (5.64) and (7.10b) and the fact that $\boldsymbol{\sigma}_{\alpha} \in L^{\infty}\left(0, T ;\left[L^{2}(\mathcal{D})\right]_{S P D}^{2 \times 2}\right)$.

It follows from (5.3), (7.10a,b), (7.11a-d), (7.12a), (3.10b), (6.12) and (3.13) that we may pass to the limit, $h, \Delta t \rightarrow 0_{+}$, in the $L$-independent version of (5.62) to obtain that $\left(\boldsymbol{u}_{\alpha}, \boldsymbol{\sigma}_{\alpha}\right)$ satisfy (7.13a). It also follows from (5.28a), (5.3), (7.11c d) and as $\mathrm{V}$ is dense in $\mathrm{H}$ that $\boldsymbol{u}_{\alpha}(0, \cdot)=\boldsymbol{u}^{0}(\cdot)$ in the required sense; see (7.13c) and Lemma 1.4 on p179 in Temam. Tem84

It follows from (7.12a c), (7.11d), (6.10), (7.10a b), 6.1a b), (1.4a) and as $\boldsymbol{u}_{\alpha} \in$ $L^{2}(0, T ; \mathrm{V})$ that we may pass to the limit $h, \Delta t \rightarrow 0_{+}$in the $L$-independent version of (5.63) with $\chi=\pi_{h} \phi$ to obtain (7.13b) for any $\phi \in C_{0}^{\infty}\left(-T, T ;\left[C^{\infty}(\overline{\mathcal{D}})\right]_{S}^{2 \times 2}\right)$. For example, in order to pass to the limit on the first and third terms in the $L$ independent version of (5.63), we note that

$$
\begin{aligned}
& \int_{0}^{T} \int_{\mathcal{D}} \pi_{h} {\left[\left(\frac{\partial \boldsymbol{\sigma}_{\alpha, h}^{\Delta t}}{\partial t}+\frac{1}{\mathrm{Wi}} \boldsymbol{\sigma}_{\alpha, h}^{\Delta t,+}\right): \pi_{h} \boldsymbol{\phi}\right] d t } \\
&=\int_{0}^{T} \int_{\mathcal{D}}\left[\frac{1}{\mathrm{Wi}} \boldsymbol{\sigma}_{\alpha, h}^{\Delta t,+}: \pi_{h} \boldsymbol{\phi}-\boldsymbol{\sigma}_{\alpha, h}^{\Delta t}: \pi_{h}\left[\frac{\partial \boldsymbol{\phi}}{\partial t}\right]\right] d t-\int_{D} \pi_{h}\left[\boldsymbol{\sigma}_{\alpha, h}^{\Delta t}: \pi_{h} \boldsymbol{\phi}\right](0, \cdot) \\
& \quad+\int_{0}^{T} \int_{\mathcal{D}}\left(\pi_{h}-\boldsymbol{I}\right)\left[\frac{1}{\mathrm{Wi}} \boldsymbol{\sigma}_{\alpha, h}^{\Delta t,+}: \pi_{h} \boldsymbol{\phi}-\boldsymbol{\sigma}_{\alpha, h}^{\Delta t}: \pi_{h}\left[\frac{\partial \boldsymbol{\phi}}{\partial t}\right]\right] d t
\end{aligned}
$$




$$
\begin{aligned}
& \int_{\mathcal{D}} \boldsymbol{\nabla} \boldsymbol{u}_{\alpha, h}^{\Delta t,+}: \pi_{h}\left[\boldsymbol{\sigma}_{\alpha, h}^{\Delta t,+} \pi_{h} \boldsymbol{\phi}\right] \\
& =\int_{\mathcal{D}} \boldsymbol{\nabla} \boldsymbol{u}_{\alpha, h}^{\Delta t,+}:\left(\pi_{h}-\boldsymbol{I}\right)\left[\boldsymbol{\sigma}_{\alpha, h}^{\Delta t,+} \pi_{h} \boldsymbol{\phi}\right] \\
& \quad-\int_{\mathcal{D}}\left\{\left(\left(\nabla \pi_{h} \phi\right) \boldsymbol{u}_{\alpha, h}^{\Delta t,+}\right): \boldsymbol{\sigma}_{\alpha, h}^{\Delta t,+}+\boldsymbol{u}_{\alpha, h}^{\Delta,+} \cdot\left(\left(\pi_{h} \phi\right) \operatorname{div} \boldsymbol{\sigma}_{\alpha, h}^{\Delta t,+}\right)\right\}
\end{aligned}
$$

where

$$
\left(\left(\nabla \pi_{h} \phi\right) \boldsymbol{u}_{\alpha, h}^{\Delta t,+}\right)(t, \boldsymbol{x})
$$
$\epsilon$ lows from noting that $C_{0}^{\infty}\left(-T, T ;\left[C^{\infty}(\overline{\mathcal{D}})\right]_{S}^{2 \times 2}\right)$ is dense in $W_{0}^{1,1}\left(0, T ;\left[H^{1}(\mathcal{D})\right]_{S}^{2 \times 2}\right.$ फ

We have the analogue of Remark 6.1.

Remark 7.1. It follows from (7.10a,b), (7.11a,b) and (7.12a,b) that

$$
\begin{aligned}
& \sup _{t \in(0, T)}\left\|\boldsymbol{u}_{\alpha}\right\|_{L^{2}(\mathcal{D})}^{2}+\int_{0}^{T}\left\|\nabla \boldsymbol{u}_{\alpha}\right\|_{L^{2}(\mathcal{D})}^{2} d t \leq C, \\
& \sup _{t \in(0, T)}\left\|\boldsymbol{\sigma}_{\alpha}\right\|_{L^{2}(\mathcal{D})}^{2}+\alpha \int_{0}^{T}\left\|\boldsymbol{\nabla} \boldsymbol{\sigma}_{\alpha}\right\|_{L^{2}(\mathcal{D})}^{2} d t \leq C\left(\alpha^{-1}, T\right) .
\end{aligned}
$$

Hence, although we have introduced diffusion with a positive coefficient $\alpha$ into the stress equation (7.13b) compared to the standard Oldroyd-B model; the bound (7.15a) on the velocity $\boldsymbol{u}_{\alpha}$ is independent of the parameter $\alpha$, where $\left(\boldsymbol{u}_{\alpha}, \boldsymbol{\sigma}_{\alpha}\right)$ solves $\left(\mathrm{P}_{\alpha}\right),(7.13 \mathrm{a}-\mathrm{c})$.

\section{Acknowledgement}

This work was initiated, whilst the authors were visiting the Beijing International Center for Mathematical Research. We would like to thank Professor Pingwen Zhang for his kind hospitality.

\section{Bibliography}

BB09. J. W. Barrett and S. Boyaval. Existence and approximation of a (regularized) FENE-P model. 2009. (in preparation).

BF92. F. Brezzi and M. Fortin. Mixed and Hybrid Finite Element Methods. SpringerVerlag, New York, 1992.

BLM09. S. Boyaval, T. Lelièvre, and C. Mangoubi. Free-energy-dissipative schemes for the Oldroyd-B model. ESAIM: Mathematical Modelling and Numerical Analysis, 43(3):523-561, may 2009.

BN04. J. W. Barrett and R. Nürnberg. Convergence of a finite-element approximation of surfactant spreading on a thin film in the presence of van der Waals forces. IMA J. Numer. Anal., 24:323-363, 2004.

Bof97. Daniele Boffi. Three-dimensional finite element methods for the stokes problem. SIAM J. Numer. Anal., 34:664-670, 1997.

BS07. J. W. Barrett and E. Süli. Existence of global weak solutions to some regularized kinetic models for dilute polymers. Multiscale Model. Simul., 6:506-546, 2007. 
BS08. J. W. Barrett and E. Süli. Existence of global weak solutions to dumbbell models for dilute polymers with microscopic cut-off. Math. Models Methods Appl. Sci., 18:935-971, 2008.

BS10. J. W. Barrett and E. Süli. Finite element approximation of kinetic dilute polymer models with microscopic cut-off. ESAIM: Mathematical Modelling and Numerical Analysis, 2010. (to appear).

EG04. A. Ern and J. L. Guermond. Theory and Practice of Finite Elements. Springer Verlag, New York, 2004.

FCGO02. E. Fernádez-Cara, G. Guillén, and R.R. Ortega. Handbook of Numerical Analysis, Vol. VIII, chapter Mathematical modelling and analysis of viscoelastic fluids of the Oldroyd kind, pages 543-661. Elsevier, 2002.

FK05. R. Fattal and R. Kupferman. Time-dependent simulation of visco-elastic flows at high weissenberg number using the log-conformation representation. J. NonNewtonian Fluid Mech., 126:23-27, 2005.

GR86. V. Girault and P.-A. Raviart. Finite Element Methods for Navier-Stokes Equations. Berlin, 1986.

GR00. G. Grün and M. Rumpf. Nonnegativity preserving numerical schemes for the thin film equation. Numer. Math., 87:113-152, 2000.

GS90. C. Guillopé and J.-C. Saut. Existence results for the flow of viscoelastic fluids with a differential constitutive law. Nonlinear Analysis, 15:849-869, 1990.

HL07. D. Hu and T. Lelièvre. New entropy estimates for Oldroyd-B and related models. Commun. Math. Sci., 5:909-916, 2007.

HR82. J. G. Heywood and R. Rannacher. Finite element approximation of the nonstationary Navier-Stokes problem I: Regularity of solutions and second-order error estimates for spatial discretization. SIAM J. Numer. Anal., 19:275-311, 1982.

JLLO06. B. Jourdain, C. Le Bris, T. Lelièvre, and F. Otto. Long-time asymptotics of a multiscale model for polymeric fluid flows. Archive for Rational Mechanics and Analysis, 181:97-148, 2006.

LLZ08. Z. Lei, C. Liu, and Y. Zhou. Global solutions for incompressible viscoelastic fluids. Archive for Rational Mechanics and Analysis, 188:371-398, 2008.

LM00. P.-L. Lions and N. Masmoudi. Global solutions for some Oldroyd models of non-Newtonian flows. Chin. Ann. Math. Ser. B, 21:131-146, 2000.

LX06. Y.-J. Lee and J. Xu. New formulations, positivity preserving discretizations and stability ananlysis for non-Newtonian flow models. Comput. Methods Appl. Mech. Engrg., 195:1180-1206, 2006.

SB95. R. Sureshkumar and A.N. Beris. Effect of artificial stress diffusivity on the stability of numerical calculations and the flow dynamics of time-dependent viscoelastic flows. J. Non-Newtonian Fluid Mech., 60:53-80, 1995.

Sch06. J. D. Schieber. Generalized Brownian configuration field for Fokker-Planck equations including center-of-mass diffusion. J. Non-Newtonian Fluid Mech., 135:179-181, 2006.

Sim87. J. Simon. Compact sets in the space $L^{p}(0, T ; B)$. Ann. Math. Pura. Appl., 146:65-96, 1987.

Tem84. R. Temam. Navier-Stokes Equations. Theory and Numerical Analysis (Third Edition), volume 2 of Studies in Mathematics and its Applications. NorthHolland, Amsterdam, 1984. 